\title{
Refined Iwasawa theory and Kolyvagin systems of Gauss sum type
}

\author{
Masato KURIHARA
}

\section{ABSTRACT}

In this paper, we establish a refinement of the usual Iwasawa main conjecture for the ideal class groups of CM-fields over a totally real field, using higher Fitting ideals.

\section{Introduction}

In this paper, we generalize the results in our previous paper [10], and prove more refined relationship between algebraic objects and analytic objects than the usual Iwasawa main conjecture.

Suppose that $p$ is an odd prime number, and consider at first a finite abelian extension $K / \mathbf{Q}$ such that $p$ does not divide $[K: \mathbf{Q}]$. Let $\chi$ be an odd Dirichlet character of $\operatorname{Gal}(K / \mathbf{Q})$ such that the conductor of $\chi$ coincides with that of $K$. When the group of $p$-th roots of unity $\mu_{p}$ is in $K$, we assume $\chi \neq \omega$ where $\omega$ is the Teichmüller character which gives the action of $\operatorname{Gal}(K / \mathbf{Q})$ on $\mu_{p}$. Suppose that $A_{K}=C l_{K} \otimes \mathbf{Z}_{p}$ is the $p$-component of the ideal class group of $K$ and $A_{K}^{\chi}$ is the $\chi$-component of $A_{K}$ (for the precise definition, see $\S 2.2$ ). Then Mazur and Wiles [13] proved the celebrated Iwasawa main conjecture and also proved

$$
\# A_{K}^{\chi}=\# O_{\chi} / B_{1, \chi^{-1}} O_{\chi}
$$

as a corollary of the main conjecture where $O_{\chi}=\mathbf{Z}_{p}[$ Image $\chi]$ and $B_{1, \chi^{-1}}$ is the generalized Bernoulli number.

This is an equality on the orders, but we can get more information on $A_{K}^{\chi}$ as an $O_{\chi}$-module from the values of zeta functions. Using the Euler system of Gauss sums, Kolyvagin and Rubin proved an isomorphism

$$
A_{K}^{\chi} \simeq \bigoplus_{i \geq 1} \Theta_{i, K}^{(\delta), \chi} / \Theta_{i-1, K}^{(\delta), \chi}
$$

as $O_{\chi}$-modules where $\left(\Theta_{i, K}^{(\delta), \chi}\right)_{i \geq 0}$ is an increasing sequence of ideals of $O_{\chi}, \Theta_{0, K}^{(\delta), \chi}=B_{1, \chi^{-1}} O_{\chi}$, and $\Theta_{i, K}^{(\delta), \chi}$, s are determined by some arguments of Euler systems from some Stickelberger elements (Kolyvagin [8] Theorem 7 and Rubin [16] Theorem 4.4; in [16] only the case $K=$ $\mathbf{Q}\left(\mu_{p}\right)$ was studied but the same argument works for $K$ with $\left.p \nmid[K: \mathbf{Q}]\right)$. For the precise definition of these ideals $\Theta_{i, K}^{(\delta), \chi}$, see $\S 7$.

In our previous paper [10], we generalized the above result to a finite and abelian extension $K / k$ such that $p$ does not divide $[K: k]$ where $k$ is a totally real base field and $K$ is a CM-field. We also assume $\chi \neq \omega$ when $\mu_{p}$ is in $K$. We obtained an isomorphism (Theorem 0.1 in [10])

$$
A_{K}^{\chi} \simeq \bigoplus_{i \geq 1} \Theta_{i, K / k}^{\chi} / \Theta_{i-1, K / k}^{\chi}
$$

as $O_{\chi}$-modules (under certain mild assumption on $\chi$ ), using an increasing sequence $\left(\Theta_{i, K / k}^{\chi}\right)_{i \geq 0}$ of ideals of $O_{\chi}$. The ideals $\Theta_{i, K / k}^{\chi}$ are determined by some Stickelberger elements over $k$, so 
determined by some analytic information coming from zeta values. (We can also define $\Theta_{i, K / k}^{(\delta), \chi}$ by the argument of Euler systems over $k$, and in our case $\Theta_{i, K / k}^{(\delta), \chi}=\Theta_{i, K / k}^{\chi}$ holds. The ideal $\Theta_{i, K / k}^{\chi}$ is better than $\Theta_{i, K / k}^{(\delta), \chi}$ for numerical computations. For the definition of these ideals, see $\S 7$.) More precisely, an idea in [10] was to use the higher Fitting ideals (for the definition, see $\S 8)$, and we proved in $[\mathbf{1 0}]$ that

$$
\operatorname{Fitt}_{i, O_{\chi}}\left(A_{K}^{\chi}\right)=\Theta_{i, K / k}^{\chi}
$$

for all $i \geq 0$ where the left hand side is the $i$-th Fitting ideal. The equality (0.4) immediately implies the isomorphism (0.3). In the following, we fix a totally real base field $k$, and omit $k$ from the notation and write $\Theta_{i, K}^{\chi}$ for $\Theta_{i, K / k}^{\chi}$.

In this paper, we generalize the above result (0.4), and study the Iwasawa theoretic version. Let $K / k$ and $\chi$ be as above. We consider the Iwasawa module $X_{K_{\infty}}=\lim A_{K_{m}}$ for the cyclotomic $\mathbf{Z}_{p}$-extension $K_{\infty}$ of a CM-field $K\left(K_{m}\right.$ is the $m$-th layer of $\left.\overleftarrow{K}_{\infty} / K\right)$, and the $\chi$-component $X_{K_{\infty}}^{\chi}$. The Iwasawa main conjecture proved by Mazur and Wiles [13] in the case $k=\mathbf{Q}$ and by Wiles $[\mathbf{2 3}]$ in general is the Iwasawa theoretic version of (0.1), and can be stated as

$$
\operatorname{Fitt}_{0, \Lambda}\left(X_{K_{\infty}}^{\chi}\right)=\left(\theta_{K_{\infty}}^{\chi}\right)
$$

since $\operatorname{Fitt}_{0, \Lambda}\left(X_{K_{\infty}}^{\chi}\right)$ is equal to the characteristic ideal of $X_{K_{\infty}}^{\chi}$ in this case (cf. Theorem 8.6 and Lemma 8.1). Here, $\Lambda=\mathbf{Z}_{p}\left[\left[\operatorname{Gal}\left(K_{\infty} / k\right)\right]\right]^{\chi}$ is the $\chi$-component of $\mathbf{Z}_{p}\left[\left[\operatorname{Gal}\left(K_{\infty} / k\right)\right]\right]$, and $\theta_{K_{\infty}}^{\chi}$ is the projective limit of the $\chi$-component $\theta_{K_{m}}^{\chi}$ of the Stickelberger element of $K_{m}$ (see $\S 2.4$ and $\S 8.3)$ and is essentially the $p$-adic $L$-function of Deligne and Ribet [2]. In this paper, we study the higher Fitting ideal $\operatorname{Fitt}_{i, \mathbf{Z}_{p}\left[\left[\operatorname{Gal}\left(K_{\infty} / k\right)\right]\right]}\left(X_{K_{\infty}}^{\chi}\right)$ for any $i \geq 0$, and will prove that it is equal to some higher Stickelberger ideal $\Theta_{i, K_{\infty}}^{\chi}$ (see $\S 7$ for the definition) which is generated by some elements coming from the $p$-adic $L$-function. Our main theorem is Theorem 1.1 in $\S 1$, which is stated as

$$
\operatorname{Fitt}_{i, \Lambda}\left(X_{K_{\infty}}^{\chi}\right)=\Theta_{i, K_{\infty}}^{\chi}
$$

for any $i \geq 0$. The case $i=0$ of Theorem 1.1 is nothing but (0.5), so our Theorem 1.1 is a refinement of the usual main conjecture. We mention here that we do not give a new proof of the main conjecture because we use the main conjecture as an important ingredient of the proof.

Our Theorem 1.1 is regarded as the Iwasawa theoretic version of the structure theorem (0.3), so is also a generalization of $(0.2)$ by Kolyvagin and Rubin [16] for abelian fields over $\mathbf{Q}$. We will also obtain another structure theorem, see Corollary 1.4 .

An essential difference in our case from [10] is that we have to work over group rings while we worked over discrete valuation rings in [10]. A key new ingredient is Kolyvagin systems of Gauss sum type, especially those which do not come from Euler systems.

More precisely, the key of the proof of our main theorem is the construction of some element $x_{\mathfrak{n}, \mathfrak{l}}\left(\mathrm{cf} . \S 7\right.$ ) in the multiplicative group having good properties (for the key property of $x_{\mathfrak{n}, \mathfrak{l}}$, see Lemma 9.2). The essential ingredient of $x_{\mathfrak{n}, \mathfrak{l}}$ is the Kolyvagin system $\kappa_{\mathfrak{n}, \mathfrak{l}}$ of Gauss sum type.

The notion of Kolyvagin systems was introduced by Mazur and Rubin in [12]. The first important property is " $\kappa_{n} \in H_{\mathcal{F}(n)}^{1}$ " in the terminology of Mazur and Rubin (cf. [12] Definition 3.1.3), which had not been recognized before [12]. More important and beautiful property of our Kolyvagin system $\kappa_{\mathfrak{n}, \mathfrak{l}}$ is that they are related to the values of $L$-functions (see the properties (ii) and (iv) below). Without explaining the notation, we gather here the properties of $\kappa_{\mathfrak{n}, \mathfrak{l}}$. We prove (under the assumption that $\kappa_{\mathfrak{n}, \mathfrak{l}}$ is defined and $\mathfrak{n} \mathfrak{l}$ is well-ordered; see Propositions $4.2,5.4,5.5$ and also Corollary 5.2 for the details) 
(i) For each prime $\mathfrak{r}$ dividing $\mathfrak{n}, \operatorname{div}_{\mathfrak{r}}\left(\kappa_{\mathfrak{n}, \mathfrak{l}}\right)=\bar{\phi}_{\mathfrak{r}}\left(\kappa_{\frac{\mathfrak{n}}{\mathfrak{r}}, \mathfrak{l}}\right)$,

(ii) $\operatorname{div}_{\mathfrak{l}}\left(\kappa_{\mathfrak{n}, \mathfrak{l}}\right)=\delta_{\mathfrak{n}}$,

(iii) For each prime $\mathfrak{r}$ dividing $\mathfrak{n}, \bar{\phi}_{\mathfrak{r}}\left(\kappa_{\mathfrak{n}, \mathfrak{l}}\right)=0$,

(iv) $\bar{\phi}_{\mathfrak{l}}\left(\kappa_{\mathfrak{n}, \mathfrak{l}}\right)=-\delta_{\mathfrak{n} \mathfrak{l}}$.

Here, $\bar{\phi}_{\mathfrak{l}}$ is defined from the reciprocity map (see $\S 2.3$ for the definition), and $\delta_{\mathfrak{n}}, \delta_{\mathfrak{n} \mathfrak{l}}$ are defined from the values of $L$-functions. The property (i) is a usual property of Kolyvagin systems (Euler systems), and the property (iii) corresponds to " $\kappa_{n} \in H_{\mathcal{F}(n)}^{1}$ " in the terminology of Mazur and Rubin. The properties (ii) and (iv) are new, and are beautiful relations between the $L$-values and the Kolyvagin system of Gauss sum type.

The idea in this paper can be applied to more general case, namely to the Iwasawa theory for more general $p$-adic representations, for example, for elliptic curves (see [11]). In this paper, we study only the minus class groups because this case is the most typical and simplest case in this theory.

In $\S 1$, we state our main theorem. In $\S 2$, we fix notation in this paper and prove basic lemmas. We review in $\S 3$ the Euler system of Gauss sum type in [10]. Suppose that $k$ is a totally real number field, and $K$ is a CM-field such that $K / k$ is finite and abelian. For a prime $\mathfrak{l}$ which splits completely in $K$, we consider the Euler system $g_{\mathfrak{l}}^{K}$ of Gauss sum type constructed in [10]. This element $g_{\mathfrak{l}}^{K}$ is related to the values of $L$-functions, namely the image of $g_{\mathfrak{l}}^{K}$ under the "divisor" map is related to $L$-values by definition (see $\S 3.2$ ), and we prove that the image of $g_{\mathfrak{l}}^{K}$ under the reciprocity map of local class field theory is also related to $L$-values (see Proposition 5.1). In $\S 3$, we also prove the congruence relation (Proposition 3.2) which is not trivial since our Euler system is a "finite" Euler system (see $\S 3.2)$. Using some abelian extension $K(\mathfrak{n}) / k$, we can define the Kolyvagin derivative $\kappa_{\mathfrak{n}, \mathfrak{l}}\left(\in K^{\times} /\left(K^{\times}\right)^{p^{N}}\right)$ from $g_{\mathfrak{l}}^{K(\mathfrak{n})}$ by the usual argument of Euler systems if the prime $\mathfrak{l}$ splits completely in $K(\mathfrak{n})$. But we need $\kappa_{\mathfrak{n}, \mathfrak{l}}$ for more general $\mathfrak{l}$ which does not necessarily split in $K(\mathfrak{n})$. In $\S 4$, we construct $\kappa_{\mathfrak{n}, \mathfrak{l}}$ for more general primes $\mathfrak{l}$, and prove the above properties (i)-(iv) in $\S 5$. We introduce in $\S 6$ the element $x_{\mathfrak{n}, \mathfrak{l}}$ which plays an important role in the proof of Theorem 1.1. In $\S 7$, we define two higher Stickelberger ideals $\Theta_{i, K}^{(\delta), \chi}$ and $\Theta_{i, K}^{\chi}$. The former is related to the theory of Euler systems, but the latter is better in general (cf. Remark 7.2). In $\S 8$, after we gather known facts on Fitting ideals, we prove that $\Theta_{i, K_{\infty}}^{\chi}$ is in the higher Fitting ideal of the Iwasawa module (Corollary 8.12). In $\S 9$, we prove Theorem 1.1. We also give some numerical examples in Remark 9.5.

I would like to express my sincere gratitude to late Professor Iwasawa for his interest in this work when I explained to him the first version of the theory in this paper. I would also like to thank K. Kato heartily for his interest in this work, and for giving me an opportunity of a series of lectures on this subject at Kyoto University in 2007. I would like to thank K. Rubin very much for his interesting lectures I attended in 2002 on Kolyvagin systems. I thank K. Kurano very much for telling me an example in Remark 8.4, and M. Aoki for the discussions on the subject in this paper. I am very grateful to D. Burns, J. Coates, R. Greenberg and C. Greither for helpful discussions with them on the subjects related to this work. I finally thank very much the referee for his careful reading and suggestions.

Notation. For an abelian group $A$ and an integer $n, A[n]$ (resp. $A / n$ ) denotes the kernel (resp. cokernel) of the multiplication by $n$. The notation $A / n$ will be used even for multiplicative groups. For example, for the multiplicative group $K^{\times}$of a field $K, K^{\times} / n$ means $K^{\times} /\left(K^{\times}\right)^{n}$. For a group $G$ and a $G$-module $M, M^{G}$ denotes the $G$-invariant part of $M$ (the maximal subgroup of $M$ on which $G$ acts trivially), and $M_{G}$ denotes the $G$-coinvariant of $M$ (the 
maximal quotient of $M$ on which $G$ acts trivially). For a prime number $p$, we denote by $\operatorname{ord}_{p}$ the additive discrete valuation of $\mathbf{Q}$ associated to $p$, which is normalized such that $\operatorname{ord}_{p}(p)=1$. For a positive integer $n, \mu_{n}$ denotes the group of all $n$-th roots of unity in an algebraic closure of the field we are considering. For a number field or a local field $F, O_{F}$ denotes the ring of integers.

\section{Main Result}

Throughout this paper, $k$ is the base field which is a totally real number field of finite degree over $\mathbf{Q}$. We assume $p$ is an odd prime number, and suppose that $K_{0}$ is a CM-field such that $K_{0} / k$ is finite and abelian, and $\left[K_{0}: k\right]$ is prime to $p$. In this $\S 1$, we denote by $K_{\infty}$ the cyclotomic $\mathbf{Z}_{p}$-extension of $K_{0}$. (In $\S 2-\S 7$, we consider more general $K$, and $K_{\infty}$ will denote the cyclotomic $\mathbf{Z}_{p}$-extension of $K$.) We put $X_{K_{\infty}}=\lim _{\leftarrow} A_{K_{0, m}}$ where $A_{K_{0, m}}$ is the $p$ component of the ideal class group of $K_{0, m}$ for the intermediate field $K_{0, m}$ of $K_{\infty} / K_{0}$ such that $\left[K_{0, m}: K_{0}\right]=p^{m}$. This $\mathbf{Z}_{p}\left[\left[\operatorname{Gal}\left(K_{\infty} / k\right)\right]\right]$-module $X_{K_{\infty}}$ which is isomorphic to the Galois group of the maximal unramified abelian pro- $p$ extension of $K_{\infty}$ is often called the Iwasawa module.

Since $\left[K_{0}: k\right]$ is prime to $p, X_{K_{\infty}}$ is decomposed into the character components for characters of $\operatorname{Gal}\left(K_{0} / k\right)$ (see $\S 2$, subsection 2.2). Let $\chi$ be an odd character of $\operatorname{Gal}\left(K_{0} / k\right)$. When $k\left(\mu_{p}\right) \subset K_{0}$, we assume $\chi \neq \omega$ where $\omega$ is the Teichmüller character which gives the action of $\operatorname{Gal}\left(K_{0} / k\right)$ on the group of $p$-th roots of unity. We also assume that the conductor of $\chi$ is equal to the conductor of $K_{0} / k$, and consider the $\chi$-component $X_{K_{\infty}}^{\chi}$ (see $\S 2.2$ ) which is a $\mathbf{Z}_{p}\left[\left[\mathrm{Gal}\left(K_{\infty} / k\right)\right]\right]^{\chi}$-module. (When we are interested in the $\chi$-component, we may change $K_{0}$ such that the conductor of $K_{0} / k$ equals that of $\chi$, cf. subsection 2.2.) We put $\Lambda=\mathbf{Z}_{p}\left[\left[\operatorname{Gal}\left(K_{\infty} / k\right)\right]\right]^{\chi}=O_{\chi}\left[\left[\operatorname{Gal}\left(K_{\infty} / K_{0}\right)\right]\right]$ where $O_{\chi}=\mathbf{Z}_{p}[$ Image $\chi]$.

Let $\theta_{K_{\infty}}^{\chi}$ be the projective limit of the $\chi$-component $\theta_{K_{0, m}}^{\chi}$ of the Stickelberger element of $K_{0, m}$ (see $\S 2.4$ and $\S 8.3$ ). As we explained in $\S 0$, the main conjecture states that the characteristic ideal of $X_{K_{\infty}}^{\chi}$ is generated by $\theta_{K_{\infty}}^{\chi}$. In this paper, we prove that more information on the structure of $X_{K_{\infty}}^{\chi}$ can be derived from the $p$-adic zeta functions, more precisely from the Stickelberger elements of abelian extensions which contain $K_{\infty}$. In $\S 7$, we define the higher Stickelberger ideals $\Theta_{i, K_{0, m}}^{\chi}$ for any $i \geq 0$ and $m \geq 0$, using the Stickelberger elements of several fields $L$ which contain $K_{0, m}$ such that $L / k$ is finite and abelian. We define the Stickelberger ideal $\Theta_{i, K_{\infty}}^{\chi} \subset \Lambda$ of $K_{\infty}$ to be the projective limit of $\Theta_{i, K_{0, m}}^{\chi}$ (see $\S 8.5$ ). In particular, $\Theta_{0, K_{\infty}}^{\chi}$ is a principal ideal generated by $\theta_{K_{\infty}}^{\chi}\left(\Theta_{i, K_{\infty}}^{\chi}\right.$ 's for $i \geq 1$ are not principal ideals, in general).

To state our main theorem, we use higher Fitting ideals (see $\S 8$ for the definition and the basic properties of higher Fitting ideals). Our main theorem is

Theorem 1.1. We assume that the $\mu$-invariant of $X_{K_{\infty}}^{\chi}$ is zero (namely, $X_{K_{\infty}}^{\chi}$ is finitely generated over $\mathbf{Z}_{p}$ ), and that $\chi(\mathfrak{p}) \neq 1$ for any prime $\mathfrak{p}$ of $k$ above $p$. Then we have

$$
\operatorname{Fitt}_{i, \Lambda}\left(X_{K_{\infty}}^{\chi}\right)=\Theta_{i, K_{\infty}}^{\chi}
$$

for all $i \geq 0$.

REMARK 1.2. (1) The left hand side of the above equation is an algebraic object and the right hand side is a $p$-adic analytic object. The above theorem gives more refined relationship between them than the usual main conjecture.

(2) If we know all Fitting ideals $\operatorname{Fitt}_{i, \Lambda}\left(X_{K_{\infty}}^{\chi}\right)$, we can determine the pseudo-isomorphism class of $X_{K_{\infty}}^{\chi}$ (Lemma 8.2). So the above theorem says that the information on the $p$-adic $L$-functions determines the pseudo-isomorphism class of $X_{K_{\infty}}^{\chi}$. For example, it determines 
whether $X_{K_{\infty}}^{\chi}$ contains $\Lambda /\left(f^{2}\right)$ or $\Lambda /(f) \oplus \Lambda /(f)$ when $f^{2} \mid \theta_{K_{\infty}}^{\chi}$ for some irreducible $f \in \Lambda$, although a generator of $\operatorname{char}\left(X_{K_{\infty}}^{\chi}\right)$ is conjectured to have only simple roots.

(3) In the case $\operatorname{rank}_{O_{\chi}} X_{K_{\infty}}^{\chi} \leq 2$, if we know all $\operatorname{Fitt}_{i, \Lambda}\left(X_{K_{\infty}}^{\chi}\right)$, we can determine the isomorphism class of $X_{K_{\infty}}^{\chi}$ (Lemma 8.3). If $\operatorname{rank}_{O_{\chi}} X_{K_{\infty}}^{\chi} \geq 3$, the isomorphism class is not determined (Remark 8.4).

(4) We can remove the assumption $\chi(\mathfrak{p}) \neq 1$ in Theorem 1.1 , which will be treated in $[\mathbf{1 1}]$.

Put $R_{K_{0, m}}=\mathbf{Z}_{p}\left[\operatorname{Gal}\left(K_{0, m} / K_{0}\right)\right]^{\chi}=O_{\chi}\left[\operatorname{Gal}\left(K_{0, m} / K_{0}\right)\right]$. As a corollary of Theorem 1.1 , we prove in $\S 9.3$

TheOREm 1.3. Under the same assumption as Theorem 1.1, for any $m \geq 0$, we have

$$
\operatorname{Fitt}_{i, R_{K_{0, m}}}\left(A_{K_{0, m}}^{\chi}\right)=\Theta_{i, K_{0, m}}^{\chi}
$$

for all $i \geq 0$.

Let $\psi$ be a character of $\operatorname{Gal}\left(K_{0, m} / K_{0}\right)$ of order $p^{m}$ where $m \geq 0$. We define $O_{\chi \psi}=O_{\chi}\left[\mu_{p^{m}}\right]$. The ring homomorphism $R_{K_{0, m}} \longrightarrow O_{\chi \psi}$ induced by $\sigma \mapsto \psi(\sigma)\left(\sigma \in \operatorname{Gal}\left(K_{0, m} / K_{0}\right)\right)$ is denoted by the same letter $\psi$. We regard $O_{\chi \psi}$ as an $R_{K_{0, m}}$-module by the ring homomorphism $\psi$ : $R_{K_{0, m}} \longrightarrow O_{\chi \psi}$. We define $A_{K_{0, m}}^{\chi \psi}=A_{K_{0, m}}^{\chi} \otimes_{R_{K_{0}, m}} O_{\chi \psi}$ which is an $O_{\chi \psi}$-module. Put $\Theta_{i}^{\chi \psi}=$ $\psi\left(\Theta_{i, K_{0, m}}^{\chi}\right)$. From Theorem 1.3, we immediately have (see $\S 9.3$ )

COROLlary 1.4. There is an isomorphism

$$
A_{K_{0, m}}^{\chi \psi} \simeq \bigoplus_{i \geq 1} \Theta_{i}^{\chi \psi} / \Theta_{i-1}^{\chi \psi}
$$

of $O_{\chi \psi}$-modules.

Taking $m=0$ and $\psi=1$ in Corollary 1.4 , we obtain Theorem 0.1 in [10], which is $(0.3)$ in $\S 0$. Hence Corollary 1.4 is a generalization of $(0.2)$ and $(0.3)$ in $\S 0$.

Theorem 1.1 also says that Conjecture 8.2 in $[\mathbf{9}]$ is true. In Theorem 1.1 , the case $i=0$ is nothing but the main conjecture proved by Wiles, and the case $i=1$ can be proved by the same method as [9] Theorem 8.4 if we use the Euler system constructed in [10]. Hence what is essentially new is the case $i \geq 2$.

In the paper [9], we studied the initial Fitting ideal $\operatorname{Fitt}_{0, \mathbf{Z}_{p}\left[\left[\operatorname{Gal}\left(K_{\infty} / k\right)\right]\right]}\left(X_{K_{\infty}}\right)$ for a general CM-field $K$. In this paper, concerning the higher Fitting ideals, we only consider the case $K=K_{0, m}$ for some $m$.

\section{Notation and Preliminary Lemmas}

2.1. For a finite prime $\mathfrak{l}$ of $k$, we denote by $\kappa(\mathfrak{l})$ the residue field of $\mathfrak{l}$, and by $N(\mathfrak{l})$ the absolute norm of $\mathfrak{l}($ so $N(\mathfrak{l})=\# \kappa(\mathfrak{l}))$. We define $n_{\mathfrak{l}}$ by $n_{\mathfrak{l}}=\operatorname{ord}_{p}(N(\mathfrak{l})-1)$. We fix a positive integer $N>0$ in $\S 2-\S 6$.

LEMMA 2.1. There are infinitely many primes $\mathfrak{l}$ of degree 1 such that $n_{\mathfrak{l}} \geq N$ and that there is a cyclic extension $k(\mathfrak{l}) / k$ of degree $p^{n_{\mathfrak{r}}}$ which is unramified outside $\mathfrak{l}$ and which is totally ramified at $\mathfrak{l}$. 
We denote by $\mathcal{S}$ the set of all finite primes $\mathfrak{l}$ of $k$ which satisfy the conditions of Lemma 2.1. If $p$ divides the class number of $k, k(\mathfrak{l})$ is not unique. But we will take a $k(\mathfrak{l})$ satisfying the above conditions for each prime $\mathfrak{l} \in \mathcal{S}$, and fix it throughout this paper.

Correction: In [10] Lemma 4.3, it is stated that there exists a unique such extension, but clearly we do not have the uniqueness if $k$ has an unramified abelian extension of degree $p$. The word "unique" in the statement in [10] Lemma 4.3 should be deleted.

Proof of Lemma 2.1. Suppose that the p-primary component $A_{k}$ of the ideal class group of $k$ is generated as an abelian group by the classes of prime ideals $\mathfrak{q}_{1}, \ldots, \mathfrak{q}_{s}$. Suppose that the order of the class $\left[\mathfrak{q}_{j}\right]$ in $A_{k}$ is $p^{a_{j}}$. We take $\xi_{j} \in k^{\times}$such that $\mathfrak{q}_{j}^{p_{j}}=\left(\xi_{j}\right)$ for each $j$. We denote by $\mathcal{U}$ the subgroup of $k^{\times}$generated by the unit group $E_{k}=O_{k}^{\times}$and $\xi_{1}, \ldots, \xi_{s}$.

We take $n$ sufficiently large such that $n \geq N$ and $k\left(\mu_{p^{n}}\right) \neq k\left(\mu_{p^{n+1}}\right)$. The Galois group $\operatorname{Gal}\left(k\left(\mu_{p}\right) / k\right)$ acts on $\operatorname{Gal}\left(k\left(\mu_{p^{n+1}}\right) / k\left(\mu_{p^{n}}\right)\right)$ trivially, and on $\operatorname{Gal}\left(k\left(\mu_{p^{n}}, \mathcal{U}^{1 / p^{n}}\right) / k\left(\mu_{p^{n}}\right)\right)$ via $\omega$ where $\omega: \operatorname{Gal}\left(k\left(\mu_{p}\right) / k\right) \longrightarrow \mathbf{Z}_{p}^{\times}$is the Teichmüller character which gives the action on $\mu_{p}$. Hence $k\left(\mu_{p^{n+1}}\right) / k\left(\mu_{p^{n}}\right)$ and $k\left(\mu_{p^{n}}, \mathcal{U}^{1 / p^{n}}\right) / k\left(\mu_{p^{n}}\right)$ are linearly disjoint, and $k\left(\mu_{p^{n}}, \mathcal{U}^{1 / p^{n}}\right) \neq$ $k\left(\mu_{p^{n+1}}, \mathcal{U}^{1 / p^{n}}\right)$. We take a prime $\mathfrak{l}$ of $k$ of degree 1 , which is prime to $p \mathfrak{q}_{1} \cdot \ldots \cdot \mathfrak{q}_{s}$, which splits completely in $k\left(\mu_{p^{n}}, \mathcal{U}^{1 / p^{n}}\right)$, and which does not split completely in $k\left(\mu_{p^{n+1}}, \mathcal{U}^{1 / p^{n}}\right)$. By the Chebotarev density theorem, there are infinitely many such $\mathfrak{l}$ 's. We will show that $\mathfrak{l}$ satisfies the conditions of Lemma 2.1.

First of all, since $\mathfrak{l}$ splits in $k\left(\mu_{p^{n}}\right)$ and does not split in $k\left(\mu_{p^{n+1}}\right)$, it is clear that $n_{\mathfrak{l}}=n$.

Let $H_{k}$ be the $p$-Hilbert class field of $k$ (hence $A_{k} \simeq \operatorname{Gal}\left(H_{k} / k\right)$ ), and $k\{\mathfrak{l}\}$ be the maximal $p$-extension of $k$ in the ray class field mod $\mathfrak{l}$. We know by class field theory $\operatorname{Gal}\left(k\{\mathfrak{l}\} / H_{k}\right) \simeq$ $\left(\kappa(\mathfrak{l})^{\times} /\left(E_{k} \bmod \mathfrak{l}\right)\right) \otimes \mathbf{Z}_{p}$ where $\left(E_{k} \bmod \mathfrak{l}\right)$ is the image of $E_{k}$ in $\kappa(\mathfrak{l})^{\times}$. Since $\mathfrak{l}$ splits completely in $k\left(E_{k}^{1 / p^{n}}\right)$ and $n=n_{\mathfrak{l}}$, we have $\left(E_{k} \bmod \mathfrak{l}\right)=\{1\}$, and $\operatorname{Gal}\left(k\{\mathfrak{l}\} / H_{k}\right) \simeq \mathbf{Z} / p^{n_{\mathfrak{l}}}$.

Furthermore, since $\mathfrak{l}$ splits completely in $k\left(\mathcal{U}^{1 / p^{n}}\right)$, by class field theory we can show that the sequence

$$
0 \longrightarrow \operatorname{Gal}\left(k\{\mathfrak{l}\} / H_{k}\right) \longrightarrow \operatorname{Gal}(k\{\mathfrak{l}\} / k) \longrightarrow \operatorname{Gal}\left(H_{k} / k\right) \longrightarrow 0
$$

splits as an exact sequence of abelian groups (see the proof of Lemma 4.3 in [10]). This shows that $k$ has a cyclic extension of degree $p^{n_{\mathfrak{l}}}$, which is unramified outside $\mathfrak{l}$ and which is totally ramified at $\mathfrak{l}$.

2.2. Suppose that $K / k$ is a finite and abelian extension, and $K$ is a CM-field (hence $K$ is totally imaginary and there is an intermediate field $K^{+}$of $K / k$ such that $K^{+}$is totally real, and $\left.\left[K: K^{+}\right]=2\right)$. We write $\operatorname{Gal}(K / k)=\Delta(K / k) \times \Gamma(K / k)$ where the order of $\Delta(K / k)$ is prime to $p$, and $\Gamma(K / k)$ is a $p$-group.

Suppose that $\chi: \Delta(K / k) \longrightarrow \overline{\mathbf{Q}}_{p}^{\times}$is a character of $\Delta(K / k)$ whose values are in an algebraic closure of $\mathbf{Q}_{p}$. For a $\mathbf{Z}_{p}[\operatorname{Gal}(K / k)]$-module $M$, we define $M^{\chi}$ by

$$
M^{\chi}=M \otimes \mathbf{z}_{p}[\Delta(K / k)] O_{\chi}
$$

where $O_{\chi}=\mathbf{Z}_{p}[$ Image $\chi]$ is the $\mathbf{Z}_{p}[\Delta(K / k)]$-module on which $\Delta(K / k)$ acts via $\chi$. Since we can also write $M^{\chi}=M \otimes_{\mathbf{z}_{p}[\operatorname{Gal}(K / k)]} O_{\chi}[\Gamma(K / k)]$, it is an $O_{\chi}[\Gamma(K / k)]$-module. For any element $x \in M$, we denote by $x^{\chi}$ the image of $x$ in $M^{\chi}$ (namely, $x^{\chi}=x \otimes 1$ ).

Since $\# \Delta(K / k)$ is prime to $p$, the group algebra $\mathbf{Z}_{p}[\Delta(K / k)]$ is a direct sum of discrete valuation rings, more precisely, $\mathbf{Z}_{p}[\Delta(K / k)]=\bigoplus_{\chi} O_{\chi}$ where $\chi$ runs through all $\mathbf{Q}_{p}$-conjugate classes of characters of $\Delta(K / k)$ (we say two $\overline{\mathbf{Q}}_{p}^{\times}$-valued characters $\chi_{1}$ and $\chi_{2}$ of $\Delta(K / k)$ are $\mathbf{Q}_{p^{-}}$ conjugate if $\sigma \circ \chi_{1}=\chi_{2}$ for some $\left.\sigma \in \operatorname{Gal}\left(\overline{\mathbf{Q}}_{p} / \mathbf{Q}_{p}\right)\right)$. Hence $\mathbf{Z}_{p}[\operatorname{Gal}(K / k)]=\bigoplus_{\chi} O_{\chi}[\Gamma(K / k)]$ 
and

$$
M=\bigoplus_{\chi} M^{\chi}
$$

hold for any $\mathbf{Z}_{p}[\operatorname{Gal}(K / k)]$-module $M$. Therefore, to study $M$, it suffices to study each $M^{\chi}$. Throughout this paper, we assume $\chi$ is odd. Also, when $K$ contains $\mu_{p}$, we assume $\chi \neq \omega$ where $\omega$ is the Teichmüller character.

For any number field $F$, we denote by $C l_{F}$ the ideal class group of $F$, and by $A_{F}$ the $p$ component $C l_{F} \otimes \mathbf{Z}_{p}$. For a field $K$ as above, we are interested in the $\mathbf{Z}_{p}[\operatorname{Gal}(K / k)]$-module $A_{K}=C l_{K} \otimes \mathbf{Z}_{p}$. We denote by $K_{0}$ the subfield of $K$ corresponding to $\Gamma(K / k)$ by Galois theory, hence $\operatorname{Gal}\left(K / K_{0}\right)=\Gamma(K / k)$ and $\operatorname{Gal}\left(K_{0} / k\right)=\Delta(K / k)$. Without loss of generality, we may assume the conductor of $\chi$ is equal to the conductor of $K_{0} / k$. In fact, let $\Delta_{\chi} \subset \Delta(K / k)$ be the kernel of $\chi: \Delta(K / k)=\operatorname{Gal}\left(K_{0} / k\right) \longrightarrow \overline{\mathbf{Q}}_{p}^{\times}$, and $K_{0, \chi}$ the subfield of $K_{0}$ corresponding to $\Delta_{\chi}$. We also regard $\Delta_{\chi}$ as a subgroup of $\operatorname{Gal}(K / k)=\Delta(K / k) \times \Gamma(K / k)$, and denote by $K_{\chi}$ the subfield of $K$ corresponding to $\Delta_{\chi} \subset \operatorname{Gal}(K / k)$. Since $\left[K: K_{\chi}\right]=\# \Delta_{\chi}$ is prime to $p, A_{K_{\chi}}$ is isomorphic to the $\Delta_{\chi}$-coinvariant $\left(A_{K}\right)_{\Delta_{\chi}}$ by the usual norm argument. So $A_{K_{\chi}}^{\chi}$ is isomorphic to $A_{K}^{\chi}=\left(\left(A_{K}\right)_{\Delta_{\chi}}\right)^{\chi}$. Hence, when we study $A_{K}^{\chi}$, we may regard $\chi$ as a character of $\operatorname{Gal}\left(K_{0, \chi} / k\right)$, and may assume $K_{0}=K_{0, \chi}$. So in the following, we assume the conductor of $\chi$ is equal to the conductor of $K_{0} / k$.

2.3. In this subsection, we define two important homomorphisms $\operatorname{div}_{\mathfrak{l}}$ and $\phi_{\mathrm{l}}$. Let $K$ be a field as in $\S 2.2$. We denote by $\operatorname{Div}_{K}$ the divisor group of $K$ written additively. So, an element of $\operatorname{Div}_{K}$ is of the form $\Sigma n_{i} \rho_{i}$ where $n_{i} \in \mathbf{Z}$ and $\rho_{i}$ is a finite prime of $K$. Suppose that

$$
\operatorname{div}: K^{\times} \longrightarrow \operatorname{Div}_{K}
$$

is the homomorphism which maps an element of $K^{\times}$to its principal divisor, namely for $x \in K^{\times}$, $\operatorname{div}(x)=\Sigma \operatorname{ord}_{\rho}(x) \rho \in \operatorname{Div}_{K}$ where $\operatorname{ord}_{\rho}$ is the normalized additive valuation associated to the prime ideal $\rho$.

Let $\mathcal{S}$ be the set of finite primes of $k$ defined in $\S 2.1$. For each $\mathfrak{l} \in \mathcal{S}$, we fix a prime $\mathfrak{l}_{\bar{k}}$ of an algebraic closure $\bar{k}$ above $\mathfrak{l}$ throughout this paper. For any subfield $F \subset \bar{k}$, the prime of $F$ below $\mathfrak{l}_{k}$ is denoted by $\mathfrak{l}_{F}$. So when we consider finite extensions $F_{1} / k, F_{2} / k$ such that $F_{1} \subset F_{2}$, we are always taking (and fixing) primes such that $\mathfrak{l}_{F_{2}} \mid \mathfrak{l}_{F_{1}}$.

Suppose $K \subset \bar{k}$ is as above. We define $\mathcal{S}(K)$ by

$$
\mathcal{S}(K)=\{\mathfrak{l} \in \mathcal{S} \mid \mathfrak{l} \text { splits completely in } K\} .
$$

Hence $\mathfrak{l}_{K}$ is a prime of degree 1 .

Assume that $\mathfrak{l}$ is a prime in $\mathcal{S}(K)$. We consider a map $K^{\times} \longrightarrow \bigoplus_{\rho \mid \mathfrak{r}} \mathbf{Z}$ defined by $x \mapsto$ $\Sigma_{\rho \mid \mathfrak{l}} \operatorname{ord}_{\rho}(x) \rho$. Using the fixed prime $\mathfrak{l}_{K}$ of $K$ above $\mathfrak{l}$, we regard $\bigoplus_{\rho \mid \mathfrak{l}} \mathbf{Z}$ as a free $\mathbf{Z}[\operatorname{Gal}(K / k)]$ module of rank 1 generated by $\mathfrak{l}_{K}$, and regard the above map as

$$
\operatorname{div}_{\mathfrak{l}}: K^{\times} \longrightarrow \mathbf{Z}[\operatorname{Gal}(K / k)] \text {. }
$$

Taking $\left(-\otimes \mathbf{Z} / p^{N}\right)^{\chi}$, we obtain

$$
\operatorname{div}_{\mathfrak{l}}:\left(K^{\times} / p^{N}\right)^{\chi} \longrightarrow O_{\chi} / p^{N}[\Gamma(K / k)]
$$

which we also denote by the same notation $\operatorname{div}_{\mathrm{l}}$.

We will next define $\phi_{\mathfrak{l}}$. We assume $\mathfrak{l} \in \mathcal{S}$. Recall that in $\S 2.1$ we took and fixed the field $k(\mathfrak{l})$ such that $k(\mathfrak{l}) / k$ is a cyclic extension of degree $p^{n_{\mathfrak{l}}}$ which is unramified outside $\mathfrak{l}$ and is totally ramified at $\mathfrak{l}$. We define $G_{\mathfrak{l}}$ by

$$
G_{\mathfrak{l}}=\operatorname{Gal}(k(\mathfrak{l}) / k) .
$$


Let $\tilde{\mathfrak{l}}=\mathfrak{l}_{k(\mathfrak{l})}$ be the unique prime of $k(\mathfrak{l})$ above $\mathfrak{l}$. Suppose that $k_{\mathfrak{l}}\left(\right.$ resp. $\left.k(\mathfrak{l})_{\mathfrak{l}}\right)$ is the completion of $k(\operatorname{resp} . k(\mathfrak{l}))$ at the prime $\mathfrak{l}(\operatorname{resp} . \tilde{\mathfrak{l}})$. We consider the reciprocity map

$$
\phi_{k_{\mathfrak{l}}}: k_{\mathfrak{l}}^{\times} \longrightarrow \operatorname{Gal}\left(k(\mathfrak{l})_{\mathfrak{l}} / k_{\mathfrak{l}}\right)=G_{\mathfrak{l}}
$$

of local class field theory. Since the characteristic of the residue field $\kappa(\mathfrak{l})$ of $k_{\mathfrak{l}}$ is prime to $p$, $k_{\mathfrak{l}}$ contains a primitive $p^{n_{\mathfrak{l}}}$-th root of unity. We can write $k(\mathfrak{l})_{\mathfrak{l}}=k_{\mathfrak{l}}\left(\sqrt[p]{n_{\mathfrak{l}}} \pi_{\mathfrak{l}}\right)$ for some prime element $\pi_{\mathfrak{l}}$ of $k_{\mathfrak{l}}$. We identify $G_{\mathfrak{l}}$ with the group $\mu_{p^{n_{\mathfrak{l}}}}$ of $p^{n_{\mathfrak{l}}}$-th roots of unity by

$$
\operatorname{Kum}: G_{\mathfrak{l}} \stackrel{\simeq}{\longrightarrow} \mu_{p^{n_{\mathfrak{l}}}}, \quad \sigma \mapsto(\sigma-1)\left(\sqrt[n_{\mathfrak{l}}]{\pi_{\mathfrak{l}}}\right) .
$$

Note that $(\sigma-1)\left(\sqrt[p]{n_{\mathfrak{l}}} \pi_{\mathfrak{l}}\right)$ means, of course, $\sigma\left(\sqrt[n_{\mathfrak{l}}]{\pi_{\mathfrak{l}}}\right) / p_{\mathfrak{l}}^{n_{\mathfrak{l}}} \pi_{\mathfrak{l}}$, and that this map does not depend on the choice of $\pi_{\mathfrak{l}}$. We have

$$
\operatorname{Kum} \circ \phi_{k_{\mathfrak{r}}}(u)=\bar{u}^{\frac{1-N(\mathfrak{r})}{p^{n_{\mathfrak{r}}}}} \in \mu_{p^{n_{\mathfrak{r}}}}
$$

(Serre [20] Chap.XIV Proposition 6 and Corollaire to Proposition 8) for all units $u \in U_{k_{\mathrm{r}}}=O_{k_{\mathrm{r}}}^{\times}$ where $N(\mathfrak{l})=\# \kappa(\mathfrak{l})$ is the absolute norm $(\kappa(\mathfrak{l})$ is the residue field of $\mathfrak{l}), \bar{u}=u \bmod \mathfrak{l} \in \kappa(\mathfrak{l})$, and we regard here $\mu_{p^{n_{\mathfrak{l}}}}$ as a subgroup of $\kappa(\mathfrak{l})^{\times}$. The extension $k(\mathfrak{l})_{\mathfrak{l}} / k_{\mathfrak{l}}$ is tamely ramified, and the above map is known as the tame symbol. (Note that some authors are using the inverse of our $\phi_{k_{\mathrm{l}}}$ as the reciprocity map.)

Lemma 2.2. Let $\phi_{k_{\mathfrak{l}},(N)}: k_{\mathfrak{l}}^{\times} \longrightarrow G_{\mathfrak{l}} \otimes \mathbf{Z} / p^{N}$ be $\phi_{k_{\mathfrak{l}}} \bmod p^{N}$. Suppose that $\beta \in k(\mathfrak{l})_{\tilde{\mathfrak{l}}}^{\times}, \sigma \in$ $\operatorname{Gal}\left(k(\mathfrak{l})_{\mathfrak{l}} / k_{\mathfrak{l}}\right)=G_{\mathfrak{l}}$, and that

$$
(\sigma-1) \beta=\frac{\sigma(\beta)}{\beta} \equiv u^{\frac{1-N(\mathfrak{l})}{p^{N}}}(\bmod \tilde{\mathfrak{l}})
$$

holds for some $u \in U_{k_{\mathrm{r}}}=O_{k_{\mathrm{l}}}^{\times}$. Then we have

$$
\phi_{k_{\mathfrak{l}},(N)}(u)=\sigma^{p^{N} \operatorname{ord}_{\mathfrak{l}}(\beta)}
$$

where we extended to $k(\mathfrak{l})_{\mathfrak{l}}$ the normalized additive valuation $\operatorname{ord}_{\mathfrak{l}}$ of $k_{\mathfrak{l}}$.

Proof. Let

$$
\operatorname{Kum}_{(N)}: G_{\mathfrak{l}} \otimes \mathbf{Z} / p^{N} \stackrel{\simeq}{\longrightarrow} \mu_{p^{N}}
$$

be the $\bmod p^{N}$ of the homomorphism Kum, namely $\sigma \mapsto(\sigma-1)\left(\sqrt[p^{N}]{\pi_{\mathfrak{l}}}\right)$. Then by $(2.1)$ we have

$$
\operatorname{Kum}_{(N)} \circ \phi_{k_{\mathfrak{r}},(N)}(u)=\bar{u}^{\frac{1-N(\mathfrak{r})}{p^{N}}} .
$$

Since

$$
u^{\frac{1-N(\mathfrak{l})}{p^{N}}} \equiv \frac{\sigma(\beta)}{\beta} \equiv\left(\frac{\sigma\left(\sqrt[p]{N} \pi_{\mathfrak{l}}\right.}{\beta}\right)^{p^{N}} \sqrt{\pi_{\mathfrak{l}}} \operatorname{ord}_{\mathfrak{l}}(\beta) \equiv \frac{\sigma^{p^{N} \operatorname{ord}_{\mathfrak{l}}(\beta)}\left(\sqrt[p]{\pi_{\mathfrak{l}}}\right)}{\sqrt[p^{N}]{\pi_{\mathfrak{l}}}}
$$

we obtain $\phi_{k_{\mathfrak{l}},(N)}(u)=\sigma^{p^{N} \operatorname{ord}_{\mathfrak{l}}(\beta)}$.

By the definition of $\mathcal{S}$ and local class field theory, we know that $k_{\mathfrak{l}}^{\times} / p^{N}=k_{\mathfrak{l}}^{\times} /\left(k_{\mathfrak{l}}^{\times}\right)^{p^{N}}$ is a direct sum of the kernels of $\operatorname{ord}_{\mathfrak{l}}$ and $\phi_{k_{\mathfrak{l}}}$. More precisely, we have the following lemma, immediately.

LEMMA 2.3. $k_{\mathfrak{l}}^{\times} / p^{N}$ is a free $\mathbf{Z} / p^{N}$-module of rank 2. We define $V_{1}$ (resp. $V_{2}$ ) to be the kernel of the map $\operatorname{ord}_{\mathfrak{l},(N)}: k_{\mathfrak{l}}^{\times} / p^{N} \longrightarrow \mathbf{Z} / p^{N}$ which is the normalized additive valuation mod $p^{N}$ (resp. of the map $\phi_{k_{\mathfrak{l}},(N)}: k_{\mathfrak{l}}^{\times} / p^{N} \longrightarrow G_{\mathfrak{l}} \otimes \mathbf{Z} / p^{N}$ which is $\phi_{k_{\mathfrak{l}}} \bmod p^{N}$ ). Then both $V_{1}$ and 
$V_{2}$ are free of rank 1 over $\mathbf{Z} / p^{N}$ and

$$
k_{\mathfrak{l}}^{\times} / p^{N}=V_{1} \oplus V_{2} .
$$

Furthermore, $V_{2}$ is the image of $\left(k(\mathfrak{l})_{\tilde{\mathfrak{l}}}^{(N)}\right)^{\times} \stackrel{N_{\mathfrak{l}}^{(N)}}{\longrightarrow} k_{\mathfrak{l}}^{\times} \longrightarrow k_{\mathfrak{l}}^{\times} / p^{N}$ where $k(\mathfrak{l})_{\mathfrak{l}}^{(N)}$ is the intermediate field of degree $p^{N}$ of $k(\mathfrak{l})_{\mathfrak{l}} / k_{\mathfrak{l}}$, and $N_{\mathfrak{l}}^{(N)}$ is the norm map of $k(\mathfrak{l})_{\tilde{\mathfrak{l}}}^{(N)} / k_{\mathfrak{l}}$.

Suppose that $\mathfrak{l}$ is in $\mathcal{S}(K)$. Since $\mathfrak{l}$ splits completely in $K$, the natural inclusion map $k \longrightarrow K$ induces an isomorphism $k_{\mathfrak{l}} \longrightarrow K_{\rho}$ for any prime $\rho$ of $K$ above $\mathfrak{l}$ where $K_{\rho}$ is the completion of $K$ at $\rho$. We consider the reciprocity map $K_{\rho}^{\times} \longrightarrow G_{\mathfrak{l}}$. We define $\phi_{\mathfrak{l}}$ by the composition

$$
\phi_{\mathfrak{l}}: K^{\times} \longrightarrow \bigoplus_{\rho \mid \mathfrak{r}} K_{\rho}^{\times} \longrightarrow \bigoplus_{\rho \mid \mathfrak{l}} G_{\mathfrak{l}}=\left(\bigoplus_{\rho \mid \mathfrak{l}} \mathbf{Z}\right) \otimes G_{\mathfrak{l}} \simeq \mathbf{Z}[\operatorname{Gal}(K / k)] \otimes G_{\mathfrak{l}}
$$

where the first map is the diagonal inclusion, the second map consists of the reciprocity maps, and the third isomorphism is defined by the identification of $\bigoplus_{\rho \mid \mathrm{r}} \mathbf{Z}$ with $\mathbf{Z}[\operatorname{Gal}(K / k)]$ using $\mathfrak{l}_{K}$. This map $\phi_{\mathrm{l}}$ is a $\mathbf{Z}[\mathrm{Gal}(K / k)]$-linear homomorphism.

Again, taking $\left(-\otimes \mathbf{Z} / p^{N}\right)^{\chi}$, we obtain

$$
\phi_{\mathfrak{l}}:\left(K^{\times} / p^{N}\right)^{\chi} \longrightarrow O_{\chi} / p^{N}[\Gamma(K / k)] \otimes G_{\mathfrak{l}}
$$

which we also denote by the same letter $\phi_{\mathfrak{l}}$. When we fix a generator $\sigma_{\mathfrak{l}}$ of $G_{\mathfrak{l}}$, we have a non-canonical isomorphism $O_{\chi} / p^{N}[\Gamma(K / k)] \otimes G_{\mathfrak{l}} \simeq O_{\chi} / p^{N}[\Gamma(K / k)]$, and we define $\bar{\phi}_{\mathfrak{l}}$ to be the composition of $\phi_{\mathfrak{l}}$ with this isomorphism

$$
\bar{\phi}_{\mathrm{l}}:\left(K^{\times} / p^{N}\right)^{\chi} \longrightarrow O_{\chi} / p^{N}[\Gamma(K / k)] .
$$

Namely, $\phi_{\mathfrak{l}}(x)=\bar{\phi}_{\mathfrak{l}}(x) \otimes \sigma_{\mathfrak{l}}$ for all $x$.

We put $K(\mathfrak{l})=K k(\mathfrak{l})$. We remark that if $x \in\left(K^{\times} / p^{N}\right)^{\chi}$ is a norm from $K(\mathfrak{l})$ (namely $x$ can be written as $x=N_{K(\mathfrak{l}) / K}(y)$ for some $y \in\left(K(\mathfrak{l})^{\times} / p^{N}\right)^{\chi}$ where $N_{K(\mathfrak{l}) / K}$ is the norm map), we have $\phi_{\mathrm{l}}(x)=0$ by local class field theory and the definition of $\phi_{\mathrm{l}}$.

2.4. In this subsection, we define the Stickelberger element for an abelian extension. Let $K / k$ be a finite and abelian extension. For a non-zero ideal $\mathfrak{a}$ of $O_{K}$, we denote by $(\mathfrak{a}, K / k)$ the Artin symbol. We define the partial zeta function for $\sigma \in \operatorname{Gal}(K / k)$ by

$$
\zeta(s, \sigma)=\sum_{(\mathfrak{a}, K / k)=\sigma} N(\mathfrak{a})^{-s}
$$

for $\operatorname{Re}(s)>1$ where $N(\mathfrak{a})$ is the absolute norm and $\mathfrak{a}$ runs through all non-zero integral ideals which are prime to the ramified primes in $K / k$. The equivariant zeta function $\theta_{K / k}(s)$ is defined by

$$
\theta_{K / k}(s)=\sum_{\sigma \in \operatorname{Gal}(K / k)} \zeta(s, \sigma) \sigma^{-1} .
$$

Suppose that $L / k$ is a finite and abelian extension such that $K \subset L$. The natural restriction $\operatorname{map} \operatorname{Gal}(L / k) \longrightarrow \operatorname{Gal}(K / k)$ induces

$$
c_{L / K}: \mathbf{C}[\operatorname{Gal}(L / k)] \longrightarrow \mathbf{C}[\operatorname{Gal}(K / k)] .
$$

Using the fact that $\theta_{K / k}(s)$ and $\theta_{L / k}(s)$ have the Euler products (Tate [21] Proposition 1.6), we can show that

$$
c_{L / K}\left(\theta_{L / k}(s)\right)=\left(\prod_{\mathfrak{l} \in R_{L / K}}\left(1-N(\mathfrak{l})^{-s} \varphi_{\mathfrak{l}}^{-1}\right)\right) \theta_{K / k}(s)
$$

where $R_{L / K}$ is the set of finite primes of $k$ which are ramified in $L$ and which are unramified in $K$, and $\varphi_{\mathfrak{r}}$ is the Frobenius of $\mathfrak{l}$ in $\operatorname{Gal}(K / k)$ (cf. Tate $[\mathbf{2 1}]$ p.86). 
The partial zeta functions have meromorphic continuation for the whole complex plane, and we know by Klingen and Siegel that $\theta_{K / k}(0)$ is in $\mathbf{Q}[\operatorname{Gal}(K / k)]([\mathbf{1 9}])$. We simply write $\theta_{K}$ for $\theta_{K / k}(0)$. By the above formula, we have

$$
c_{L / K}\left(\theta_{L}\right)=\left(\prod_{\mathfrak{l} \in R_{L / K}}\left(1-\varphi_{\mathfrak{r}}^{-1}\right)\right) \theta_{K} .
$$

Let $K$ be as in $\S 2.2$. We consider the $\chi$-component $\mathbf{Z}_{p}[\operatorname{Gal}(K / k)]^{\chi}=O_{\chi}[\Gamma(K / k)]$ of the group ring. As in $\S 2.1$, we assume $\chi \neq \omega$. Consider the natural map $\mathbf{Q}[\operatorname{Gal}(K / k)]=\mathbf{Q}[\Delta(K / k) \times$ $\Gamma(K / k)] \longrightarrow \mathbf{Q}_{p}($ Image $\chi)[\Gamma(K / k)]$ defined by $\Sigma a_{\sigma, \tau}(\sigma, \tau) \mapsto \Sigma a_{\sigma, \tau} \chi(\sigma) \tau$. We define $\theta_{K}^{\chi} \in$ $\mathbf{Q}_{p}$ (Image $\left.\chi\right)[\Gamma(K / k)]$ to be the image of $\theta_{K}$ by this map (see $\S 2.2$ for the general definition of the element $x^{\chi}$ for general $x$ ). Since we are assuming $\chi \neq \omega$, we have

$$
\theta_{K}^{\chi} \in O_{\chi}[\Gamma(K / k)]
$$

by Deligne and Ribet [2]. We note that this element $\theta_{K}^{\chi}$ is numerically computable in principle.

Suppose that $L / k$ is finite and abelian such that $K \subset L$ and $L / K$ is a $p$-extension. In the notation of $\S 2.2$, we have $\Delta(K / k)=\Delta(L / k)$, and we can define $\theta_{L}^{\chi} \in O_{\chi}[\Gamma(L / k)]$. By the above equation, we have the following lemma which will be used many times.

Lemma 2.4. Let $c_{L / K}: O_{\chi}[\Gamma(L / k)] \longrightarrow O_{\chi}[\Gamma(K / k)]$ be the restriction map. Then we have

$$
c_{L / K}\left(\theta_{L}^{\chi}\right)=\left(\prod_{\mathfrak{r} \in R_{L / K}}\left(1-\varphi_{\mathfrak{r}}^{-1}\right)^{\chi}\right) \theta_{K}^{\chi} .
$$

\section{Euler systems of Gauss sum type}

In this section, we review the Euler system of Gauss sum type in [10], and prove some fundamental properties.

3.1. From now on, we always assume the following. We consider a number field $K$ as in $\S 2.2$, namely $K$ is a CM-field such that $K / k$ is finite and abelian. We use the same notation $\Delta(K / k)$, $\Gamma(K / k), K_{0}$ (recall that $K_{0}$ is the field such that $\operatorname{Gal}\left(K / K_{0}\right)=\Gamma(K / k)$ ), and consider an odd character $\chi$ of $\Delta(K / k)$. As in $\S 2.2$, we assume $\chi \neq \omega$, and the conductor of $\chi$ is equal to that of $K_{0}$. We also assume that $\chi(\mathfrak{p}) \neq 1$ for all primes $\mathfrak{p}$ of $k$ above $p$, and the $\mu_{\chi}$-invariant of $K$ is zero. The second assumption means the following. For the cyclotomic $\mathbf{Z}_{p}$-extension $K_{\infty} / K$, we define $X_{K_{\infty}}$ by $X_{K_{\infty}}=\lim A_{K_{n}}$ where $K_{n}$ is the intermediate field of degree $p^{n}$, and the limit is taken with respect to the norm maps. The assumption that the $\mu_{\chi}$-invariant of $K$ vanishes means $\mu\left(X_{K_{\infty}}^{\chi}\right)=0$, namely the $\chi$-component $X_{K_{\infty}}^{\chi}$ is a finitely generated $O_{\chi}$-module (this is always true by a famous theorem of Ferrero and Washington if $k=\mathbf{Q}[\mathbf{3}])$. We consider such general $K$ in $\S 3-\S 7$ (we do not assume $K \subset K_{0, \infty}$ ). Furthermore, in $\S 3-\S 6$ we also assume that

$\left.{ }^{*}\right)$ all primes of $k$ above $p$ are ramified in $K$, and all primes of $K$ above $p$ are totally ramified in $K_{\infty}$.

3.2. We next review the result in $[\mathbf{1 0}] \S 4$. We consider abelian $p$-extensions $L / K$, more precisely, put

$$
\mathcal{F}=\{L \mid K \subset L, L / k \text { is finite and abelian, and } L / K \text { is a } p \text {-extension }\},
$$

and consider $L \in \mathcal{F}$. Note that $\operatorname{Gal}(L / k)=\Delta(L / k) \times \Gamma(L / k), \Gamma(L / k)=\operatorname{Gal}\left(L / K_{0}\right)$, and $\Delta(L / k)=\Delta(K / k)=\operatorname{Gal}\left(K_{0} / k\right)$ (recall that $K_{0}$ is the field such that $K / K_{0}$ is a $p$-extension 
and $\left[K_{0}: K\right]$ is prime to $\left.p\right)$. Note that $L$ is a CM-field because $K$ is a CM-field, $L / K$ is a $p$-extension $(p \neq 2)$ and $L / k$ is an abelian extension.

We regard $\chi$ as a character of $\Delta(L / k)$, and consider the $\chi$-components $\left(L^{\times} \otimes \mathbf{Z}_{p}\right)^{\chi}$, $\left(\operatorname{Div}_{L} \otimes \mathbf{Z}_{p}\right)^{\chi}$, etc. Let $A_{L}^{\chi}$ be the $\chi$-component of the $p$-component of the ideal class group of $L$. We denote by $O_{L}^{\times}\left(\operatorname{resp} . \operatorname{Div}_{L}\right)$ the unit group of $L$ (resp. the divisor group of $L$ ). As in $\S 2.3$, we define div : $L^{\times} \longrightarrow \operatorname{Div}_{L}$ to be the homomorphism which maps an element of $L^{\times}$to its principal divisor. Then we have an exact sequence $0 \longrightarrow O_{L}^{\times} \otimes \mathbf{Z}_{p} \longrightarrow L^{\times} \otimes \mathbf{Z}_{p} \stackrel{\text { div }}{\longrightarrow}$ $\operatorname{Div}_{L} \otimes \mathbf{Z}_{p} \longrightarrow A_{L} \longrightarrow 0$. Taking the $\chi$-component, we obtain

Lemma 3.1. For $L \in \mathcal{F}$,

$$
0 \longrightarrow\left(L^{\times} \otimes \mathbf{Z}_{p}\right)^{\chi} \stackrel{\operatorname{div}}{\longrightarrow}\left(\operatorname{Div}_{L} \otimes \mathbf{Z}_{p}\right)^{\chi} \longrightarrow A_{L}^{\chi} \longrightarrow 0
$$

is exact.

Proof. This follows from $\left(O_{L}^{\times} \otimes \mathbf{Z}_{p}\right)^{\chi}=0$ which can be easily checked by our assumption that $\chi$ is odd and $\chi \neq \omega$.

We use the same notation as in $\S 2.3$ for $L$. Recall that $\mathcal{S}(L)$ is the subset of finite primes of $k$ defined by

$$
\mathcal{S}(L)=\{\mathfrak{l} \in \mathcal{S} \mid \mathfrak{l} \text { splits completely in } L\},
$$

and we consistently fixed a prime $\mathfrak{l}_{L}$ of $L$ above $\mathfrak{l}$ for each $\mathfrak{l} \in \mathcal{S}(L)$.

By Corollary 2.4 in [10] (note that the $\mu$-invariant of $L$ vanishes because of our assumption of the vanishing of the $\mu$-invariant of $K$ and the fact that $L / K$ is a $p$-extension (Theorem 2 in Iwasawa [6])), we have

$$
\theta_{L}^{\chi} A_{L}^{\chi}=0
$$

For $\mathfrak{l} \in \mathcal{S}(L)$, the class of $\mathfrak{l}_{L}$ in $A_{L}^{\chi}$ is denoted by $\left[\mathfrak{l}_{L}\right]^{\chi}$. Then $\theta_{L}^{\chi}\left[\mathfrak{l}_{L}\right]^{\chi}=0$ holds. By the exact sequence in Lemma 3.1, there is a unique element $g_{\mathfrak{l}}^{L}$ in $\left(L^{\times} \otimes \mathbf{Z}_{p}\right)^{\chi}$ such that

$$
\operatorname{div}\left(g_{\mathfrak{l}}^{L}\right)=\theta_{L}^{\chi} \mathfrak{l}_{L}^{\chi}
$$

(note that $\mathfrak{l}_{L}^{\chi}$ is the image of $\mathfrak{l}_{L}$ in $\left.\left(\operatorname{Div}_{L} \otimes \mathbf{Z}_{p}\right)^{\chi}\right)$.

Suppose that $M$ is a subfield such that $K_{0} \subset M \subset L$ (where $K_{0}$ is the subfield such that $\operatorname{Gal}\left(K_{0} / k\right)=\Delta(K / k)$ as in $\left.\S 2.2\right)$. Recall that we are taking $\mathfrak{l}_{M}$ such that $\mathfrak{l}_{L} \mid \mathfrak{l}_{M}$, so we can define $g_{\mathfrak{l}}^{M}$ similarly. Using Lemma 2.4 , if $\mathfrak{l} \in \mathcal{S}(L)$, namely if $\mathfrak{l} \in \mathcal{S}$ splits completely in $L$, we have (see [10] Lemma 4.1)

$$
N_{L / M}\left(g_{\mathfrak{l}}^{L}\right)=\left(\prod_{\rho \in R_{L / M}}\left(1-\varphi_{\rho}^{-1}\right)^{\chi}\right)\left(g_{\mathfrak{l}}^{M}\right)
$$

where $N_{L / M}$ is the norm map and $R_{L / M}$ is defined similarly for $L / M$ as in $\S 2.4$. Note that if $\mathfrak{l}$ does not split completely in $L,(3.2)$ does not hold (the residue degree appears in the formula cf. Lemma 4.1 in [10]). Thus, for $\mathfrak{l} \in \mathcal{S}(L)$, for any intermediate field $M$ of $L / K_{0}$, we obtain an Euler system $\left(g_{\mathfrak{l}}^{M}\right)$. But this is a "finite" Euler system in the terminology of Mazur and Rubin [12] because it is defined only on the finite set $\left\{M \mid K_{0} \subset M \subset L\right\}$. For more details for this Euler system, see $[\mathbf{1 0}] \S 4$.

3.3. In this subsection, we recall the usual argument of Euler systems to construct the Kolyvagin derivative $\kappa_{\mathfrak{n}, \mathfrak{l}}$. Recall at first that in $\S 2.1$ for each $\mathfrak{r} \in \mathcal{S}$ we took and fixed a field $k(\mathfrak{r})$ such that $k(\mathfrak{r}) / k$ is a cyclic extension of degree $p^{n_{\mathfrak{r}}}$, which is unramified outside $\mathfrak{r}$ and is totally ramified at $\mathfrak{r}$. We define $G_{\mathfrak{r}}$ by $G_{\mathfrak{r}}=\operatorname{Gal}(k(\mathfrak{r}) / k)$. As in the usual argument of Euler 
systems, taking a generator $\sigma_{\mathfrak{r}}$ of $G_{\mathfrak{r}}$ we put

$$
N_{\mathfrak{r}}=\sum_{i=0}^{p^{n_{\mathfrak{r}}}-1} \sigma_{\mathfrak{r}}^{i} \in \mathbf{Z}\left[G_{\mathfrak{r}}\right] \text { and } D_{\mathfrak{r}}=\sum_{i=0}^{p^{n_{\mathfrak{r}}}-1} i \sigma_{\mathfrak{r}}^{i} \in \mathbf{Z}\left[G_{\mathfrak{r}}\right] .
$$

A fundamental equation is $D_{\mathfrak{r}}\left(\sigma_{\mathfrak{r}}-1\right)=p^{n_{\mathfrak{r}}}-N_{\mathfrak{r}}$.

We define $\mathcal{N}$ (resp. $\mathcal{N}(K))$ to be the set consisting of all squarefree products of primes in $\mathcal{S}$ $\left(\right.$ resp. $\mathcal{S}(K)$ ) (we denote by 1 the ideal $(1)=O_{k}$ and suppose 1 is both in $\mathcal{N}$ and $\mathcal{N}(K)$ ). For any $\mathfrak{n} \in \mathcal{N}$ with $\mathfrak{n}=\mathfrak{r}_{1} \cdot \ldots \cdot \mathfrak{r}_{m}$, define $k(\mathfrak{n})$ to be the compositum of the fields $k\left(\mathfrak{r}_{1}\right), \ldots, k\left(\mathfrak{r}_{m}\right)$, and $G_{\mathfrak{n}}=G_{\mathfrak{r}_{1}} \times \ldots \times G_{\mathfrak{r}_{m}}$ which is isomorphic to $\operatorname{Gal}(k(\mathfrak{n}) / k)$.

For $\mathfrak{n} \in \mathcal{N}(K)$, we write $K(\mathfrak{n})=K k(\mathfrak{n})$. Clearly, we have $\operatorname{Gal}(K(\mathfrak{n}) / K)=\operatorname{Gal}(k(\mathfrak{n}) / k)=G_{\mathfrak{n}}$. Note that $K(\mathfrak{n}) \in \mathcal{F}$ where $\mathcal{F}$ is the set defined in $\S 3.2$. We put $N_{\mathfrak{n}}=\Pi_{\mathfrak{r} \mid \mathfrak{n}} N_{\mathfrak{r}}$ and $D_{\mathfrak{n}}=\Pi_{\mathfrak{r} \mid \mathfrak{n}} D_{\mathfrak{r}}$ which are elements of $\mathbf{Z}\left[G_{\mathfrak{n}}\right]$.

We use the standard argument of Euler systems $([\mathbf{1 5}] \S 2,[\mathbf{2 2}] \S 15.3)$. For $\mathfrak{l} \in \mathcal{S}(K(\mathfrak{n}))$, $D_{\mathfrak{n}} g_{\mathfrak{l}}^{K(\mathfrak{n})} \bmod p^{N}$ is in the $G_{\mathfrak{n}}$-invariant part of $\left(K(\mathfrak{n})^{\times} \otimes \mathbf{Z} / p^{N}\right)^{\chi}$. We define $\kappa_{\mathfrak{n}, \mathfrak{l}} \in\left(K^{\times} \otimes\right.$ $\left.\mathbf{Z} / p^{N}\right)^{\chi}$ to be the unique element whose image in $\left(K(\mathfrak{n})^{\times} \otimes \mathbf{Z} / p^{N}\right)^{\chi}$ is $D_{\mathfrak{n}} g_{\mathfrak{l}}^{K(\mathfrak{n})}$. The uniqueness comes from the bijectivity of the natural map

$$
\left(K^{\times} \otimes \mathbf{Z} / p^{N}\right)^{\chi} \longrightarrow\left(\left(K(\mathfrak{n})^{\times} \otimes \mathbf{Z} / p^{N}\right)^{\chi}\right)^{G_{\mathfrak{n}}},
$$

which follows from our assumption $\chi \neq \omega$. Note that $\kappa_{1, \mathfrak{l}}=g_{\mathfrak{l}}^{K}$ for $\mathfrak{n}=1$.

We next consider Stickelberger elements. Suppose that $\mathfrak{n} \in \mathcal{N}(K)$. In $\S 2.4$, we defined $\theta_{K}^{\chi} \in O_{\chi}[\Gamma(K / k)]$. We define $\theta_{K(\mathfrak{n})}^{\chi} \in O_{\chi}[\Gamma(K(\mathfrak{n}) / k)]$ by the same method. Since $\Gamma(K(\mathfrak{n}) / k)=$ $\Gamma(K / k) \times G_{\mathfrak{n}}$, we have $O_{\chi}[\Gamma(K(\mathfrak{n}) / k)]=O_{\chi}[\Gamma(K / k)]\left[G_{\mathfrak{n}}\right]$. The multiplication by $N_{\mathfrak{n}}$ gives an injective homomorphism

$$
N_{\mathfrak{n}}: O_{\chi} / p^{N}[\Gamma(K / k)] \longrightarrow O_{\chi} / p^{N}[\Gamma(K / k)]\left[G_{\mathfrak{n}}\right]
$$

of $\operatorname{Gal}(K(\mathfrak{n}) / k)$-modules, whose image is the $G_{\mathfrak{n}}$-invariant part. Since $D_{\mathfrak{n}} \theta_{K(\mathfrak{n})}^{\chi} \bmod p^{N}$ is in the $G_{\mathfrak{n}}$-invariant part of $O_{\chi} / p^{N}[\Gamma(K(\mathfrak{n}) / k)]$ (which follows from the standard argument of Euler system as above), it is in the image of $N_{\mathfrak{n}}$. Hence there is a unique element $\delta_{\mathfrak{n}}$ in $O_{\chi} / p^{N}[\Gamma(K / k)]$ such that $N_{\mathfrak{n}} \delta_{\mathfrak{n}}=D_{\mathfrak{n}} \theta_{K(\mathfrak{n})}^{\chi} \bmod p^{N}$. Note that $\delta_{1}=\theta_{K}^{\chi}$ for $\mathfrak{n}=1$.

Suppose that $\mathfrak{n}=\mathfrak{r}_{1} \cdot \ldots \cdot \mathfrak{r}_{m}$. We know

$$
\begin{aligned}
\theta_{K(\mathfrak{n})}^{\chi} \equiv(-1)^{m} \delta_{\mathfrak{n}}\left(\sigma_{\mathfrak{r}_{1}}-1\right) \cdot \ldots \cdot\left(\sigma_{\mathfrak{r}_{m}}-1\right) & \\
& \left(\bmod p^{N},\left(\sigma_{\mathfrak{r}_{1}}-1\right)^{2}, \ldots,\left(\sigma_{\mathfrak{r}_{m}}-1\right)^{2}\right)
\end{aligned}
$$

by Lemma 4.4 in $[\mathbf{1 0}]$.

Suppose again that $\mathfrak{n}=\mathfrak{r}_{1} \cdot \ldots \cdot \mathfrak{r}_{m} \in \mathcal{N}(K)$ and $\mathfrak{l} \in \mathcal{S}(K(\mathfrak{n}))$. We defined $\kappa_{\mathfrak{n}, \mathfrak{l}} \in\left(K^{\times} / p^{N}\right)^{\chi}$ above, but this does depend on the choice of a generator $\sigma_{\mathfrak{r}}$ of $G_{\mathfrak{r}}$ for each $\mathfrak{r} \mid \mathfrak{n}$. Put

$$
\mathcal{G}_{\mathfrak{n}}=G_{\mathfrak{r}_{1}} \otimes \ldots \otimes G_{\mathfrak{r}_{m}}
$$

Following Mazur and Rubin [12], we consider elements in $\left(K^{\times} / p^{N}\right)^{\chi} \otimes \mathcal{G}_{\mathfrak{n}}$, and define

$$
\tilde{\kappa}_{\mathfrak{n}, \mathfrak{l}}=\kappa_{\mathfrak{n}, \mathfrak{l}} \otimes \sigma_{\mathfrak{r}_{1}} \otimes \ldots \otimes \sigma_{\mathfrak{r}_{m}} \in\left(K^{\times} / p^{N}\right)^{\chi} \otimes \mathcal{G}_{\mathfrak{n}}
$$

which does not depend on the choice of $\sigma_{\mathfrak{r}_{i}}$. In the same way, we define

$$
\tilde{\delta_{\mathfrak{n}}}=\delta_{\mathfrak{n}} \otimes \sigma_{\mathfrak{r}_{1}} \otimes \ldots \otimes \sigma_{\mathfrak{r}_{m}} \in\left(O_{\chi} / p^{N}[\Gamma(K / k)]\right) \otimes \mathcal{G}_{\mathfrak{n}}
$$

which is also independent of the choice of $\sigma_{\mathfrak{r}_{i}}$.

3.4. We next prove a famous relation called congruence relation of Euler systems (cf. Corollary 4.8.1 in Rubin [17]). We are dealing with a "finite" Euler system, and cannot apply the usual 
argument directly, so we will give here a proof. A special case was proved and used in [10], but here we give a general version and its proof.

Proposition 3.2. (Congruence relation) Suppose $\mathfrak{r} \in \mathcal{S}$ is unramified in $K$ and $\mathfrak{l} \in \mathcal{S}(K(\mathfrak{r}))$ where $K(\mathfrak{r})=K k(\mathfrak{r})$. (Note that we do not assume $\mathfrak{r} \in \mathcal{S}(K)$.) Then, for any prime $\rho_{\mathfrak{r}}$ of $K(\mathfrak{r})$ above $\mathfrak{r}$, we have

$$
\left(g_{\mathfrak{l}}^{K}\right)^{\frac{1-N(\mathfrak{r})-1}{p^{n \mathfrak{r}}}} \equiv g_{\mathfrak{l}}^{K(\mathfrak{r})} \quad\left(\bmod \rho_{\mathfrak{r}}\right)
$$

where $N(\mathfrak{r})$ is the absolute norm $\# \kappa(\mathfrak{r})$ of $\mathfrak{r}$.

Proof. Let $\kappa\left(\rho_{\mathfrak{r}}\right)$ be the residue field of $\rho_{\mathfrak{r}}$, and put $n^{\prime}=\operatorname{ord}_{p}\left(\# \kappa\left(\rho_{\mathfrak{r}}\right)^{\times}\right)$. Obviously, $n^{\prime} \geq n_{\mathfrak{r}}$ and $n^{\prime}$ does not depend on the choice of $\rho_{\mathfrak{r}}$ and only on $K$ because $K / k$ is a Galois extension. Put $L=K(\mathfrak{r})$. By the Chebotarev density theorem, we can take a prime $\mathfrak{l}^{\prime} \in \mathcal{S}\left(L\left(\mu_{p^{n^{\prime}+n_{\mathfrak{r}}}}\right)\right)$ such that the class $\left[\mathfrak{l}_{L}^{\prime}\right]^{\chi}$ and the class $\left[\mathfrak{l}_{L}\right]^{\chi}$ coincide in $A_{L}^{\chi}$. In fact, we take $n \geq n^{\prime}+n_{\mathfrak{r}}$ sufficiently large such that $L\left(\mu_{p^{n}}\right) \neq L\left(\mu_{p^{n+1}}\right)$. Let $H_{L}$ be the maximal subfield of the Hilbert $p$-class field of $L$ such that $\Delta(L / k)$ acts on $\operatorname{Gal}\left(H_{L} / L\right)$ via $\chi$ (namely, $H_{L}$ is the subfield such that $A_{L}^{\chi} \simeq \operatorname{Gal}\left(H_{L} / L\right)$ is bijective). We also consider $\mathcal{U}$ in the proof of Lemma 2.1. Using the action of $\Delta\left(L\left(\mu_{p}\right) / k\right)$, we know $L\left(\mu_{p^{n+1}}, \mathcal{U}^{1 / p^{n}}\right) \cap H_{L}=L$ and $L\left(\mu_{p^{n}}, \mathcal{U}^{1 / p^{n}}\right) \cap L\left(\mu_{p^{n+1}}\right)=L\left(\mu_{p^{n}}\right)$. Hence we can take $\mathfrak{l}^{\prime} \in \mathcal{S}\left(L\left(\mu_{p^{n}}\right)\right)$ satisfying the above property.

We put $L^{\prime}=L\left(\mu_{p^{n^{\prime}+n_{\mathfrak{r}}}}\right)$ and $K^{\prime}=K\left(\mu_{p^{n^{\prime}+n_{\mathfrak{r}}}}\right)$. We write $\mathcal{R}\left(L^{\prime}\right)$ for $\left(\bigoplus_{v \mid \mathfrak{r}} \kappa(v)^{\times} \otimes \mathbf{Z}_{p}\right)^{\chi}$ where $\kappa(v)$ is the residue field of a prime $v$ of $L^{\prime}$ above $\mathfrak{r}$, and denote by

$$
r_{L^{\prime}}:\left(\left\{x \in L^{\prime \times} \mid(x) \text { is prime to } \mathfrak{r}\right\} \otimes \mathbf{Z}_{p}\right)^{\chi} \longrightarrow \mathcal{R}\left(L^{\prime}\right)
$$

the natural homomorphism. We define $\mathcal{R}\left(K^{\prime}\right)$ and $r_{K^{\prime}}$ similarly. Consider the images of $g_{\mathfrak{l}^{\prime}}^{K^{\prime}}$ and $g_{\mathfrak{l}^{\prime}}^{L^{\prime}}$ in $\mathcal{R}\left(L^{\prime}\right)$. Note that the natural map gives an isomorphism $\mathcal{R}\left(K^{\prime}\right) \stackrel{\simeq}{\longrightarrow} \mathcal{R}\left(L^{\prime}\right)$ because all primes of $K^{\prime}$ above $\mathfrak{r}$ are totally ramified in $L^{\prime}$. We identify $\mathcal{R}\left(L^{\prime}\right)$ with $\mathcal{R}\left(K^{\prime}\right)$ by this isomorphism. Then it follows from $\left[L^{\prime}: K^{\prime}\right]=p^{n_{\mathfrak{r}}}$ that the norm map induces the $p^{n_{\mathfrak{r}}}$-th power map on $\mathcal{R}\left(L^{\prime}\right)$. On the other hand, by the norm property of the Euler system (see (3.2) in $\S 3.2$ ) we have $N_{L^{\prime} / K^{\prime}}\left(g_{\mathfrak{l}^{\prime}}^{L^{\prime}}\right)=\left(g_{\mathfrak{l}^{\prime}}^{K^{\prime}}\right)^{\left(1-\varphi_{\mathfrak{r}}^{-1}\right)^{\chi}}$. Hence on $\mathcal{R}\left(L^{\prime}\right)$ we get $r_{L^{\prime}}\left(g_{\mathfrak{l}^{\prime}}^{L^{\prime}}\right)^{p^{n_{\mathfrak{r}}}}=r_{K^{\prime}}\left(g_{\mathfrak{l}^{\prime}}^{K^{\prime}}\right)^{1-N(\mathfrak{r})^{-1}}$. For each prime $v$ of $L^{\prime}$ above $\mathfrak{r}, \kappa(v)$ contains a primitive $p^{n^{\prime}+n_{\tau}}$ th root of unity, so we have $\operatorname{ord}_{p}\left(\# \kappa(v)^{\times}\right) \geq n^{\prime}+n_{\mathfrak{r}}$. Therefore, the above equality implies

$$
r_{L^{\prime}}\left(g_{\mathfrak{l}^{\prime}}^{L^{\prime}}\right) \equiv r_{K^{\prime}}\left(g_{\mathfrak{l}^{\prime}}^{K^{\prime}}\right)^{\frac{1-N(\mathfrak{r})-1}{p^{n_{\mathfrak{r}}}}}\left(\bmod p^{n^{\prime}}\right)
$$

(the congruence means the equality in $\mathcal{R}\left(L^{\prime}\right) / p^{n^{\prime}}$ ). Since $L^{\prime} / L$ (resp. $K^{\prime} / K$ ) is unramified outside $p$ and all primes above $p$ of $k$ are ramified in $L$ (resp. $K$ ) by the assumption $\left(^{*}\right)$ in $\S 3.1$, we have

$$
N_{L^{\prime} / L}\left(g_{\mathfrak{l}^{\prime}}^{L^{\prime}}\right)=g_{\mathfrak{l}^{\prime}}^{L} \quad\left(\text { resp. } \quad N_{K^{\prime} / K}\left(g_{\mathfrak{l}^{\prime}}^{K^{\prime}}\right)=g_{\mathfrak{l}^{\prime}}^{K}\right)
$$

by the norm property (3.2) in $§ 3.2$. Taking the norm $N_{L^{\prime} / L}$ of the both sides of the above congruence, we get

$$
r_{L}\left(g_{\mathfrak{l}^{\prime}}^{L}\right) \equiv r_{K}\left(g_{\mathfrak{l}^{\prime}}^{K}\right)^{\frac{1-N(\mathfrak{r})-1}{p^{n \mathfrak{r}}}}\left(\bmod p^{n^{\prime}}\right) .
$$

Since $\mathcal{R}(L)$ is a free $O_{\chi} / p^{n^{\prime}}$-module, the above congruence is the equality in $\mathcal{R}(L)$. This shows that Proposition 3.2 is true for $\mathfrak{l}^{\prime}$.

By our assumption on $\mathfrak{l}^{\prime}$, we can take an element $a \in\left(L^{\times} \otimes \mathbf{Z}_{p}\right)^{\chi}$ such that $\operatorname{div}(a)=$ $\left(\mathfrak{l}_{L}^{\prime}-\mathfrak{l}_{L}\right)^{\chi}$. Then we have $\operatorname{div} g_{\mathfrak{l}^{\prime}}^{L} / a^{\theta_{L}^{\chi}}=\operatorname{div} g_{\mathfrak{l}}^{L}$, which implies by Lemma 3.1 that $g_{\mathfrak{l}^{\prime}}^{L} / a^{\theta_{L}^{\chi}}=g_{\mathfrak{l}}^{L}$ in $\left(L^{\times} \otimes \mathbf{Z}_{p}\right)^{\chi}$. In the same way, putting $b=N_{L / K}(a)$, we have $\operatorname{div} g_{\mathfrak{l}^{\prime}}^{K} / b^{\theta_{K}^{\chi}}=\operatorname{div} g_{\mathfrak{l}}^{K}$, which implies $g_{\mathfrak{l}^{\prime}}^{K} / b^{\theta_{K}^{\chi}}=g_{\mathfrak{l}}^{K}$ in $\left(K^{\times} \otimes \mathbf{Z}_{p}\right)^{\chi}$ by Lemma 3.1. Since $G_{\mathfrak{r}}$ acts on $\mathcal{R}(L)$ trivially, by Lemma 
2.4 we have

$$
\begin{aligned}
r_{L}\left(a^{\theta_{L}^{\chi}}\right) & \left.=r_{L}(a)^{\left(1-\varphi_{\mathfrak{r}}^{-1}\right)^{\chi} \theta_{K}^{\chi}}=r_{L}(a)^{\theta_{K}^{\chi}\left(1-N(\mathfrak{r})^{-1}\right.}\right)=r_{L}\left(a^{p^{n_{\mathfrak{r}}}}\right)^{\theta_{K}^{\chi} \frac{1-N(\mathfrak{r})^{-1}}{p^{n \mathfrak{r}}}} \\
& =r_{K}\left(N_{L / K}(a)\right)^{\theta_{K}^{\chi} \frac{1-N(\mathfrak{r})^{-1}}{p^{n \mathfrak{r}}}}=r_{K}\left(b^{\theta_{K}^{\chi}}\right)^{\frac{1-N(\mathfrak{r})-1}{p^{n \mathfrak{r}}}}
\end{aligned}
$$

Combining (3.4) and (3.5), we get

$$
r_{L}\left(g_{\mathfrak{l}}^{L}\right)=r_{L}\left(\frac{g_{\mathfrak{l}^{\prime}}^{L}}{a^{\theta_{L}^{\chi}}}\right)=r_{K}\left(\frac{g_{\mathfrak{l}^{\prime}}^{K}}{b_{K}^{\theta_{K}^{X}}}\right)^{\frac{1-N(\mathfrak{r})^{-1}}{p^{n \mathfrak{r}}}}=r_{K}\left(g_{\mathfrak{l}}^{K}\right)^{\frac{1-N(\mathfrak{r})-1}{p^{n \mathfrak{r}}}} .
$$

This completes the proof of Proposition 3.2.

We consider a homomorphism

$$
\operatorname{div}:\left(K^{\times} / p^{N}\right)^{\chi} \otimes \mathcal{G}_{\mathfrak{n}} \longrightarrow\left(\operatorname{Div}_{K} / p^{N}\right)^{\chi} \otimes \mathcal{G}_{\mathfrak{n}}
$$

induced by the homomorphism div, and denote it also by the same notation div. For $\mathfrak{r} \in \mathcal{S}(K)$, we also use

$$
\operatorname{div}_{\mathfrak{r}}:\left(K^{\times} / p^{N}\right)^{\chi} \otimes \mathcal{G}_{\mathfrak{n}} \longrightarrow\left(O_{\chi} / p^{N}[\Gamma(K / k)]\right) \otimes \mathcal{G}_{\mathfrak{n}}
$$

which is obtained from $\operatorname{div}_{\mathfrak{r}}:\left(K^{\times} / p^{N}\right)^{\chi} \longrightarrow O_{\chi}[\Gamma(K / k)] / p^{N}$ defined in $\S 2.3$. There, we also defined

$$
\phi_{\mathfrak{r}}:\left(K^{\times} / p^{N}\right)^{\chi} \longrightarrow O_{\chi} / p^{N}[\Gamma(K / k)] \otimes \mathcal{G}_{\mathfrak{r}}
$$

for $\mathfrak{r} \in \mathcal{S}(K)$ though we used $\mathfrak{l}$ instead of $\mathfrak{r}$ in $\S 2.3$. If $\mathfrak{r}$ divides $\mathfrak{n}$, the above homomorphism induces

$$
\phi_{\mathfrak{r}}:\left(K^{\times} / p^{N}\right)^{\chi} \otimes \mathcal{G}_{\frac{\mathfrak{n}}{\mathfrak{r}}} \longrightarrow O_{\chi} / p^{N}[\Gamma(K / k)] \otimes \mathcal{G}_{\mathfrak{n}}
$$

which we also denote by $\phi_{\mathfrak{r}}$.

Proposition 3.3. Suppose that $\mathfrak{l} \in \mathcal{S}(K(\mathfrak{n}))$. Then we have

(0) If $\rho$ is a prime of $K$ which does not divide $\mathfrak{n} \mathfrak{l}$, it is not in the support of $\operatorname{div}\left(\tilde{\kappa}_{\mathfrak{n}, \mathfrak{l}}\right)$.

(1) For any prime $\mathfrak{r}$ dividing $\mathfrak{n}$, we have $\operatorname{div}_{\mathfrak{r}}\left(\tilde{\kappa}_{\mathfrak{n}, \mathfrak{l}}\right)=\phi_{\mathfrak{r}}\left(\tilde{\kappa}_{\frac{\mathfrak{n}}{\mathfrak{r}}, \mathfrak{l}}\right)$.

(2) $\operatorname{div}_{\mathfrak{l}}\left(\tilde{\kappa}_{\mathfrak{n}, \mathfrak{l}}\right)=\tilde{\delta}_{\mathfrak{n}}$.

Proof. The property (0) follows from the fact that $\rho$ is unramified in $K(\mathfrak{n})$, and $g_{\mathfrak{l}}^{K(\mathfrak{n})}$ is a unit outside $\mathfrak{l}$. The property $(2)$ is immediate from the definitions of $\tilde{\kappa}_{\mathfrak{n}, \mathfrak{l}}$ and $\tilde{\delta}_{\mathfrak{n}}$. The property (1) is a standard property of Euler systems (cf. Theorem 4.5.4 in Rubin [17]) and can be proved by the usual argument (see Proposition 2.4 in Rubin [15]). We will give here a proof to clarify where we use Proposition 3.2 and Lemma 2.2.

We take a lifting $\kappa_{\mathfrak{n}, \mathfrak{l}}^{\wedge} \in\left(K^{\times} \otimes \mathbf{Z}_{p}\right)^{\chi}$ of $\kappa_{\mathfrak{n}, \mathfrak{l}} \in\left(K^{\times} / p^{N}\right)^{\chi}$. By definition, we can write $\kappa_{\mathfrak{n}, \mathfrak{l}}^{\wedge}=$ $D_{\mathfrak{n}} g_{\mathfrak{l}}^{K(\mathfrak{n})} / \beta^{p^{N}}$ for some $\beta \in\left(K(\mathfrak{n})^{\times} \otimes \mathbf{Z}_{p}\right)^{\chi}$. In the same way, we write $\kappa_{\frac{\mathfrak{n}}{\mathfrak{r}}, \mathfrak{l}}^{\wedge}=D_{\frac{\mathfrak{n}}{\mathfrak{r}}} g_{\mathfrak{l}}^{K\left(\frac{\mathfrak{n}}{\mathfrak{r}}\right)} /\left(\beta^{\prime}\right)^{p^{N}}$ for some $\beta^{\prime} \in\left(K\left(\frac{\mathfrak{n}}{\mathfrak{r}}\right)^{\times} \otimes \mathbf{Z}_{p}\right)^{\chi}$. Since $\kappa_{\mathfrak{n}, \mathfrak{l}}^{\wedge}, \kappa_{\frac{\mathfrak{n}}{\mathfrak{r}}, \mathfrak{l}}^{\wedge}$ are elements of $\left(K^{\times} \otimes \mathbf{Z}_{p}^{\mathfrak{r}}\right)^{\chi}$, we compute

$$
\begin{aligned}
\left(\sigma_{\mathfrak{r}}-1\right) \beta & =\left(\left(\sigma_{\mathfrak{r}}-1\right) D_{\mathfrak{n}} g_{\mathfrak{l}}^{K(\mathfrak{n})}\right)^{1 / p^{N}}=\left(\left(p^{n_{\mathfrak{r}}}-N_{\mathfrak{r}}\right) D_{\frac{\mathfrak{n}}{\mathfrak{r}}} g_{\mathfrak{l}}^{K(\mathfrak{n})}\right)^{1 / p^{N}} \\
& =\left(D_{\frac{\mathfrak{n}}{\mathfrak{r}}} g_{\mathfrak{l}}^{K(\mathfrak{n})}\right)^{p_{\mathfrak{r}}^{n_{\mathfrak{r}}-N}} /\left(\left(1-\varphi_{\mathfrak{r}}^{-1}\right) D_{\frac{\mathfrak{n}}{\mathfrak{r}}} g_{\mathfrak{l}}^{K\left(\frac{\mathfrak{n}}{\mathfrak{r}}\right)}\right)^{1 / p^{N}} \\
& =\left(D_{\frac{\mathfrak{n}}{\mathfrak{r}}} g_{\mathfrak{l}}^{K(\mathfrak{n})}\right)^{p^{n_{\mathfrak{r}}-N}} /\left(1-\varphi_{\mathfrak{r}}^{-1}\right) \beta^{\prime}
\end{aligned}
$$

We apply Proposition 3.2 to $K\left(\frac{\mathfrak{n}}{\mathfrak{r}}\right)$ to get $g_{\mathfrak{l}}^{K(\mathfrak{n})} \equiv\left(g_{\mathfrak{l}}^{K\left(\frac{\mathfrak{n}}{\mathfrak{r}}\right)}\right)^{\frac{1-N(\mathfrak{r})-1}{p^{n} \mathfrak{r}}} \quad\left(\bmod \rho_{\mathfrak{r}}\right)$ for any prime $\rho_{\mathfrak{r}}$ of $K(\mathfrak{n})$ above $\mathfrak{r}$. Therefore, we have

$$
\left(\sigma_{\mathfrak{r}}-1\right) \beta \equiv\left(D_{\frac{\mathfrak{r}}{\mathfrak{r}}} g_{\mathfrak{l}}^{K\left(\frac{\mathfrak{n}}{\mathfrak{r}}\right)}\right)^{\frac{1-N(\mathfrak{r})-1}{p^{N}}} /\left(\beta^{\prime}\right)^{1-N(\mathfrak{r})^{-1}} \quad\left(\bmod \rho_{\mathfrak{r}}\right)
$$




$$
\begin{aligned}
& =\left(D_{\frac{\mathfrak{n}}{\mathfrak{r}}} g_{\mathfrak{l}}^{K\left(\frac{\mathfrak{n}}{\mathfrak{r}}\right)} /\left(\beta^{\prime}\right)^{p^{N}}\right)^{\frac{1-N(\mathfrak{r})-1}{p^{N}}}=\left(\kappa_{\frac{\mathfrak{n}}{\mathfrak{r}}, \mathfrak{l}}^{\wedge}\right)^{\frac{1-N(\mathfrak{r})-1}{p^{N}}} \\
& \equiv\left(\kappa_{\frac{\mathfrak{n}}{\mathfrak{r}}, \mathfrak{l}}^{\wedge}\right)^{\frac{N(\mathfrak{r})-1}{p^{N}}} \quad\left(\bmod \mathfrak{r}_{K}\right) .
\end{aligned}
$$

Here, we used the fact that $\varphi_{\mathfrak{r}}$ is the $N(\mathfrak{r})$-th power map on the residue field of $\rho_{\mathfrak{r}}$ to get the first congruence, and that the $N(\mathfrak{r})$-th power map is the identity map on the residue field of $\mathfrak{r}_{K}$ to get the last congruence.

By Lemma 2.2, we have $\phi_{\mathfrak{r}}\left(\kappa_{\frac{\mathfrak{n}}{\mathfrak{r}}, \mathfrak{l}}^{\wedge}\right)=\phi_{\mathfrak{r}}\left(\kappa_{\frac{\mathfrak{n}}{\mathfrak{r}}, \mathfrak{l}}\right)=\sigma_{\mathfrak{r}}^{-p^{N} \operatorname{div}_{\mathfrak{r}}(\beta)}$. Therefore, we get

$$
\phi_{\mathfrak{r}}\left(\kappa_{\mathfrak{n}}, \mathfrak{l}\right)=\sigma_{\mathfrak{r}}^{-p^{N} \operatorname{div}_{\mathfrak{r}}(\beta)}=-\operatorname{div}_{\mathfrak{r}}\left(\beta^{p^{N}}\right) \otimes \sigma_{\mathfrak{r}}=\operatorname{div}_{\mathfrak{r}}\left(\kappa_{\mathfrak{n}, \mathfrak{l}}^{\wedge}\right) \otimes \sigma_{\mathfrak{r}}=\operatorname{div}_{\mathfrak{r}}\left(\kappa_{\mathfrak{n}, \mathfrak{l}}\right) \otimes \sigma_{\mathfrak{r}}
$$

which implies $\phi_{\mathfrak{r}}\left(\tilde{\kappa}_{\frac{\mathfrak{n}}{\mathfrak{r}}, \mathfrak{l}}\right)=\operatorname{div}_{\mathfrak{r}}\left(\tilde{\kappa}_{\mathfrak{n}, \mathfrak{l}}\right)$.

REMARK 3.4. In $\S 4$ we will give more general definition of $\tilde{\kappa}_{\mathfrak{n}, \mathfrak{l}}$, for which we will prove the same properties in Proposition 4.2.

\section{Kolyvagin systems of Gauss sum type $I$}

4.1. In the argument to define $\kappa_{\mathfrak{n}, \mathfrak{r}}$ in the previous section, the assumption $\mathfrak{l} \in \mathcal{S}(K(\mathfrak{n}))$ is definitely needed because we need the norm property $(3.2)$ in $\S 3.2$ for $L=K(\mathfrak{n}), M=K$ to define $g_{\mathfrak{l}}^{K(\mathfrak{n})}$, and (3.2) holds only when $\mathfrak{l}$ splits in $L=K(\mathfrak{n})$ as we explained after (3.2). But the theory of Kolyvagin systems by Mazur and Rubin [12] suggests that there would exist $\kappa_{\mathfrak{n}, \mathfrak{l}}$ for more general $\mathfrak{l}$. They studied their theory mainly over principal ideal domains in $[\mathbf{1 2}]$, so we cannot apply it directly to our case. We will construct in this paper the elements $\kappa_{\mathfrak{n}, \mathfrak{r}}$ explicitly under some (mild) assumptions on $\mathfrak{l}$.

We will explain a little more what we need. When we define $\kappa_{\mathfrak{n}, \mathfrak{l}}, \mathfrak{l}$ has to be chosen from $\mathcal{S}(K(\mathfrak{n}))$, hence taken after we took $\mathfrak{n}$. But we need later elements $\kappa_{\mathfrak{n}, \mathfrak{l}}$ where we take $\mathfrak{n}$ after we took $\mathfrak{l}$ (see $\S 9.2$ where we define $x_{\mathfrak{n}, \mathfrak{l}}$, taking $\mathfrak{n}$ after we took $\mathfrak{l}$ ). We sometimes need both $\kappa_{\mathfrak{l}_{1}, \mathfrak{l}_{2}}$ and $\kappa_{\mathfrak{l}_{2}, \mathfrak{l}_{1}}$ (see Remark 9.6). Namely, we need $\kappa_{\mathfrak{n}, \mathfrak{l}}$ for more general $(\mathfrak{n}, \mathfrak{l})$. In the following, we will define a certain subset $\mathcal{N}_{[\epsilon(\mathfrak{n})]}(K)$ of $\mathcal{N}(K)$, and will define $\kappa_{\mathfrak{n}, \mathfrak{l}} \in\left(K^{\times} / p^{N}\right)^{\chi}$ for all $(\mathfrak{n}, \mathfrak{l})$ such that $\mathfrak{n} \mathfrak{l} \in \mathcal{N}_{[\epsilon(\mathfrak{n})]}(K)$, though it seems that $\kappa_{\mathfrak{n}, \mathfrak{l}}$ could be defined for more general (n, l) (cf. Remark 4.4 and $\$ 5.3)$.

Suppose that $c$ is the exponent of $A_{K}^{\chi}$, namely the smallest integer such that $p^{c} A_{K}^{\chi}=0$. The following lemma is easy to prove, but is useful in $\S 4.2$.

LEMma 4.1. Let $g, g^{\prime}$ be elements in $\left(K^{\times} / p^{N+c}\right)^{\chi}$. Suppose that $\operatorname{div}(g) \equiv \operatorname{div}\left(g^{\prime}\right)$ $\left(\bmod p^{N+c}\right)$. Then we have $g \bmod p^{N}=g^{\prime} \bmod p^{N}$ in $\left(K^{\times} / p^{N}\right)^{\chi}$.

Proof. Consider the exact sequence

$$
0 \longrightarrow A_{K}^{\chi}\left[p^{N+c}\right] \longrightarrow\left(K^{\times} / p^{N+c}\right)^{\chi} \longrightarrow\left(\operatorname{Div}_{K} / p^{N+c}\right)^{\chi} \longrightarrow A_{K}^{\chi} / p^{N+c} \longrightarrow 0 .
$$

Since $\left(K^{\times} \otimes \mathbf{Z}_{p}\right)^{\chi}$ is $p$-torsion free, $\left(K^{\times} / p^{N+c}\right)^{\chi}$ is a free $O_{\chi} / p^{N+c}$-module. Hence the image of $A_{K}^{\chi}\left[p^{N+c}\right]=A_{K}^{\chi}$ in $\left(K^{\times} / p^{N+c}\right)^{\chi}$ is in $p^{N}\left(K^{\times} / p^{N+c}\right)^{\chi}$. This shows that $g \bmod p^{N}=g^{\prime} \bmod$ $p^{N}$.

4.2. For any integer $m \geq N$, we define $\mathcal{S}_{m}(K)=\left\{\mathfrak{l} \in \mathcal{S}(K) \mid n_{\mathfrak{l}} \geq m\right\}$. For any integer $n>0$, we put

$$
\mathcal{S}_{[n]}(K)=\mathcal{S}_{N+n c}(K)
$$

and define $\mathcal{N}_{[n]}(K)$ to be the set consisting of all squarefree products of primes in $\mathcal{S}_{[n]}(K)$. 
For $\mathfrak{n} \in \mathcal{N}$, we define $\epsilon(\mathfrak{n})$ to be the number of primes which divide $\mathfrak{n}$ (namely, $\epsilon(\mathfrak{n})=m$ for $\mathfrak{n}=\mathfrak{r}_{1} \cdot \ldots \cdot \mathfrak{r}_{m}$ in $\left.\S 3.3\right)$, and consider $\mathcal{N}_{[\epsilon(\mathfrak{n})]}(K)$. For $\mathfrak{n}=1$, we define $\epsilon(1)=0$.

Suppose that $\mathfrak{n} \mathfrak{l} \in \mathcal{N}_{[\epsilon(\mathfrak{n})]}(K)$, and $\mathfrak{r}$ is a prime factor of $\mathfrak{n}$. Replacing $N$ by $N+\epsilon(\mathfrak{n}) c$, we can define $\phi_{\mathfrak{r}}:\left(K^{\times} / p^{N+\epsilon(\mathfrak{n}) c}\right)^{\chi} \longrightarrow O_{\chi} / p^{N+\epsilon(\mathfrak{n}) c}[\Gamma(K / k)] \otimes G_{\mathfrak{r}}$. By the same method as in the proof of Proposition 3.2, using the Chebotarev density theorem, we can take $\mathfrak{l}^{\prime} \in \mathcal{S}_{[\epsilon(\mathfrak{n})]}(K(\mathfrak{n}))$ such that the classes $\left[\mathfrak{l}_{K}^{\prime}\right]^{\chi}$ and $\left[\mathfrak{l}_{K}\right]^{\chi}$ in $A_{K}^{\chi}$ coincide. By the exact sequence in Lemma 3.1 , there is a unique element $b \in\left(K^{\times} \otimes \mathbf{Z}_{p}\right)^{\chi}$ such that $\operatorname{div}(b)=\left(\mathfrak{l}_{K}^{\prime}-\mathfrak{l}_{K}\right)^{\chi}$. Replacing $N$ by $N+\epsilon(\mathfrak{n}) c$, we define $\tilde{\delta}_{\mathfrak{n}}^{(N+\epsilon(\mathfrak{n}) c)} \in O_{\chi} / p^{N+\epsilon(\mathfrak{n}) c}[\Gamma(K / k)] \otimes G_{\mathfrak{n}}$, and $\tilde{\kappa}_{\mathfrak{n}, l^{\prime}}^{(N+\epsilon(\mathfrak{n}) c)} \in\left(K^{\times} / p^{N+\epsilon(\mathfrak{n}) c}\right)^{\chi} \otimes \mathcal{G}_{\mathfrak{n}}$ by the usual Euler system argument in $§ 3.3$. Using induction on $\epsilon(\mathfrak{n})$, we define

$$
\tilde{\kappa}_{\mathfrak{n}, \mathfrak{l}}^{\prime}=\tilde{\kappa}_{\mathfrak{n}, l^{\prime}}^{(N+\epsilon(\mathfrak{n}) c)}-\tilde{\delta}_{\mathfrak{n}}^{(N+\epsilon(\mathfrak{n}) c)} b-\sum_{\mathfrak{r} \mid \mathfrak{n}}\left(\tilde{\kappa}_{\frac{\mathfrak{r}}{\mathfrak{r}}, \mathfrak{r}}^{\prime} \otimes \phi_{\mathfrak{r}}(b)\right) \in\left(K^{\times} / p^{N+\epsilon(\mathfrak{n}) c}\right)^{\chi} \otimes \mathcal{G}_{\mathfrak{n}}
$$

Here, we wrote the group law of $\left(K^{\times} / p^{N+\epsilon(\mathfrak{n}) c}\right)^{\chi} \otimes \mathcal{G}_{\mathfrak{n}}$ additively though $K^{\times}$is a multiplicative group. The element $\tilde{\delta}_{\mathfrak{n}}^{(N+\epsilon(\mathfrak{n}) c)} b$ means $\alpha b \otimes \tau \in\left(K^{\times} / p^{N+\epsilon(\mathfrak{n}) c}\right)^{\chi} \otimes \mathcal{G}_{\mathfrak{n}}$ if $\tilde{\delta}_{\mathfrak{n}}^{(N+\epsilon(\mathfrak{n}) c)}=\alpha \otimes \tau \in$ $O_{\chi} / p^{N+\epsilon(\mathfrak{n}) c}[\Gamma(K / k)] \otimes \mathcal{G}_{\mathfrak{n}}$. The sum is taken over all primes dividing $\mathfrak{n}$. Note that $\tilde{\kappa}_{\frac{\mathfrak{n}}{\mathfrak{r}}, \mathfrak{r}}^{\prime}$ is defined by induction because of $\epsilon\left(\frac{\mathfrak{n}}{\mathfrak{r}}\right)<\epsilon(\mathfrak{n})$. We regard $\tilde{\kappa}_{\frac{\mathfrak{n}}{\mathfrak{r}}, \mathfrak{r}}^{\prime} \otimes \phi_{\mathfrak{r}}(b)$ as an element of $\left(K^{\times} / p^{N+\epsilon(\mathfrak{n}) c}\right)^{\chi} \otimes \mathcal{G}_{\mathfrak{n}}$ by the identification

$$
\begin{aligned}
& \left(\left(K^{\times} / p^{N+\epsilon(\mathfrak{n}) c}\right)^{\chi} \otimes \mathcal{G}_{\frac{\mathfrak{n}}{\mathfrak{r}}}\right) \otimes_{O_{\chi} / p^{N+\epsilon(\mathfrak{n}) c}[\Gamma(K / k)]}\left(O_{\chi} / p^{N+\epsilon(\mathfrak{n}) c}[\Gamma(K / k)] \otimes \mathcal{G}_{\mathfrak{r}}\right) \\
& =\left(K^{\times} / p^{N+\epsilon(\mathfrak{n}) c}\right)^{\chi} \otimes \mathcal{G}_{\mathfrak{n}} .
\end{aligned}
$$

We put

$$
\tilde{\kappa}_{\mathfrak{n}, \mathfrak{l}}=\tilde{\kappa}_{\mathfrak{n}, \mathfrak{l}}^{\prime} \bmod p^{N} \in\left(K^{\times} / p^{N}\right)^{\chi} \otimes \mathcal{G}_{\mathfrak{n}}
$$

Proposition 4.2. The element $\tilde{\kappa}_{\mathfrak{n}, \mathfrak{l}}$ defined above is well-defined, namely independent of the choice of $\mathfrak{l}^{\prime}$ (hence independent of the choice of $b$ ). This element satisfies the following properties.

(0) If $\rho$ is a prime of $K$ which does not divide $\mathfrak{n} \mathfrak{l}$, it is not in the support of $\operatorname{div}\left(\tilde{\kappa}_{\mathfrak{n}, l}\right)$.

(1) For any prime $\mathfrak{r}$ dividing $\mathfrak{n}$, we have $\operatorname{div}_{\mathfrak{r}}\left(\tilde{\kappa}_{\mathfrak{n}, \mathfrak{l}}\right)=\phi_{\mathfrak{r}}\left(\tilde{\kappa}_{\mathfrak{n}}^{\mathfrak{r}}, \mathfrak{l}\right)$.

(2) $\operatorname{div}_{\mathfrak{l}}\left(\tilde{\kappa}_{\mathfrak{n}, \mathfrak{l}}\right)=\tilde{\delta}_{\mathfrak{n}}$.

REMARK 4.3. In the above proposition, if we further assume that $\mathfrak{l}$ is in $\mathcal{S}(K(\mathfrak{n}))$, we can take $\mathfrak{l}^{\prime}=\mathfrak{l}$ and $b=1$. Hence we have $\phi_{\mathfrak{r}}(b)=0$, and $\tilde{\kappa}_{\mathfrak{n}, \mathfrak{l}}$ defined above coincides with $\tilde{\kappa}_{\mathfrak{n}, \mathfrak{l}}$ defined in $§ 3.3$. Therefore, our notation is consistent.

Proof. We prove this proposition by induction on $\epsilon(\mathfrak{n})$. We first show

$(0)^{\prime}$ If $\rho$ is a prime which does not divide $\mathfrak{n} \mathfrak{l}$, it is not in the support of $\operatorname{div}\left(\tilde{\kappa}_{\mathfrak{n}, \mathfrak{l}}^{\prime}\right) \bmod p^{N+c}$.

$(1)^{\prime}$ For any prime $\mathfrak{r}$ dividing $\mathfrak{n}$, we have $\operatorname{div}_{\mathfrak{r}}\left(\tilde{\kappa}_{\mathfrak{n}, \mathfrak{l}}^{\prime}\right) \equiv \phi_{\mathfrak{r}}\left(\tilde{\kappa}_{\mathfrak{n}, \mathfrak{l}}^{\prime}\right) \quad\left(\bmod p^{N+c}\right)$.

$(2)^{\prime} \operatorname{div}_{\mathfrak{l}}\left(\tilde{\kappa}_{\mathfrak{n}, \mathfrak{l}}^{\prime}\right) \equiv \tilde{\delta}_{\mathfrak{n}}^{(N+\epsilon(\mathfrak{n}) c)} \quad\left(\bmod p^{N+c}\right)$.

By induction on $\epsilon(\mathfrak{n}), N+\epsilon(\mathfrak{n}) c=N+c+\epsilon\left(\frac{\mathfrak{n}}{\mathfrak{r}}\right) c$ implies that $\tilde{\kappa}_{\frac{\mathfrak{n}}{\mathfrak{r}}, \mathfrak{r}}^{\prime} \bmod p^{N+c}$ is well-defined, namely does not depend on the choice of the auxiliary prime for $\mathfrak{r}$. Using $(0)^{\prime},(1)^{\prime},(2)^{\prime}$ for $\tilde{\kappa}_{\frac{\mathfrak{n}}{\mathfrak{r}}, \mathfrak{r}}^{\prime}$, we have

$$
\operatorname{div}\left(\tilde{\kappa}_{\frac{\mathfrak{r}}{\mathfrak{r}}, \mathfrak{r}}^{\prime}\right) \equiv \sum_{\mathfrak{r}^{\prime} \mid \mathfrak{n} \mathfrak{\mathfrak { r }}} \phi_{\mathfrak{r}^{\prime}}\left(\tilde{\kappa}_{\frac{\mathfrak{n}}{\mathfrak{r} \mathfrak{r}^{\prime}}, \mathfrak{r}}^{\prime(N+c)}\right) \mathfrak{r}_{K}^{\prime}+\tilde{\delta}_{\frac{\mathfrak{n}}{\mathfrak{r}}}^{(N+\epsilon(\mathfrak{n}) c)} \mathfrak{r}_{K} \quad\left(\bmod p^{N+c}\right) .
$$

Therefore, by Proposition $3.3(0)$, if a prime $\rho$ is prime to $\mathfrak{n} \mathfrak{l} \mathfrak{l}^{\prime}, \rho$ is not in the support of $\operatorname{div}\left(\tilde{\kappa}_{\mathfrak{n}, \mathfrak{l}}^{\prime}\right) \bmod p^{N+c}$. Concerning $\mathfrak{l}^{\prime}$, if $\mathfrak{l}^{\prime} \neq \mathfrak{l}$, by Proposition $3.3(2)$ we have

$$
\operatorname{div}_{\mathfrak{l}^{\prime}}\left(\tilde{\kappa}_{\mathfrak{n}, \mathfrak{l}}^{\prime}\right) \equiv \tilde{\delta}_{\mathfrak{n}}^{(N+\epsilon(\mathfrak{n}) c)}-\tilde{\delta}_{\mathfrak{n}}^{(N+\epsilon(\mathfrak{n}) c)} \equiv 0 \quad\left(\bmod p^{N+c}\right)
$$


Hence we get the property $(0)^{\prime}$.

For $\mathfrak{r}$ such that $\mathfrak{r} \mid \mathfrak{n}$, we can compute

$$
\begin{aligned}
\operatorname{div}_{\mathfrak{r}}\left(\tilde{\kappa}_{\mathfrak{n}, \mathfrak{l}}^{\prime}\right) & \equiv \phi_{\mathfrak{r}}\left(\tilde{\kappa}_{\frac{\mathfrak{n}}{\mathfrak{r}}, \mathfrak{l}^{\prime}}^{(N+\epsilon(\mathfrak{n}) c)}\right)-\tilde{\delta}_{\frac{\mathfrak{n}}{\mathfrak{r}}}^{(N+\epsilon(\mathfrak{n}) c)} \otimes \phi_{\mathfrak{r}}(b)-\sum_{\mathfrak{r}^{\prime} \mid \frac{\mathfrak{n}}{\mathfrak{r}}} \phi_{\mathfrak{r}}\left(\tilde{\kappa}_{\frac{\mathfrak{n}}{\mathfrak{r} \mathfrak{r}^{\prime}}, \mathfrak{r}^{\prime}}^{\prime}\right) \otimes \phi_{\mathfrak{r}^{\prime}}(b) \\
& \equiv \phi_{\mathfrak{r}}\left(\tilde{\kappa}_{\frac{\mathfrak{r}}{\mathfrak{r}}, \mathfrak{l}}^{\prime}\right) \quad\left(\bmod p^{N+c}\right),
\end{aligned}
$$

by Proposition $3.3(1)$, (2) and the definition of $\tilde{\kappa}_{\mathfrak{n}, \mathfrak{l}}^{\prime}, \tilde{\kappa}_{\frac{\mathfrak{n}}{\mathfrak{r}} \mathfrak{l} \cdot}^{\prime}$. Thus, we get the property $(1)^{\prime}$.

Concerning the property $(2)^{\prime}$, we just note that $\mathfrak{l}$ is prime to $\mathfrak{n}$, and get

$$
\operatorname{div}_{\mathfrak{l}}\left(\tilde{\kappa}_{\mathfrak{n}, \mathfrak{l}}^{\prime}\right) \equiv-\left(-\tilde{\delta}_{\mathfrak{n}}^{(N+\epsilon(\mathfrak{n}) c)}\right) \equiv \tilde{\delta}_{\mathfrak{n}}^{(N+\epsilon(\mathfrak{n}) c)} \quad\left(\bmod p^{N+c}\right)
$$

if $\mathfrak{l}^{\prime} \neq \mathfrak{l}$. If $\mathfrak{l}^{\prime}=\mathfrak{l}$, we get $\operatorname{div}_{\mathfrak{l}}\left(\tilde{\kappa}_{\mathfrak{n}, \mathfrak{l}}^{\prime}\right)=\tilde{\delta}_{\mathfrak{n}}^{(N+\epsilon(\mathfrak{n}) c)}$ by Proposition $3.3(2)$.

The properties $(0)^{\prime},(1)^{\prime},(2)^{\prime}$ imply that $\operatorname{div}\left(\tilde{\kappa}_{\mathfrak{n}, \mathfrak{l}}^{\prime}\right) \bmod p^{N+c}$ is independent of the choice of $\mathfrak{l}^{\prime}$. Hence by Lemma $4.1 \tilde{\kappa}_{\mathfrak{n}, \mathfrak{l}}=\tilde{\kappa}_{\mathfrak{n}, \mathfrak{l}}^{\prime} \bmod p^{N}$ is independent of the choice of $\mathfrak{l}^{\prime}$. This completes the proof of Proposition 4.2.

REMARK 4.4. We give another definition of $\mathcal{N}_{[\epsilon(\mathfrak{n})]}(K)$. Let $K_{\infty} / K$ be the cyclotomic $\mathbf{Z}_{p}$-extension, and $K_{m}$ the $m$-th layer. Since we assumed $\mu\left(X_{K_{\infty}}^{\chi}\right)=0$, the map $A_{K_{m}}^{\chi}\left[p^{N}\right] \longrightarrow$ $A_{K}^{\chi}\left[p^{N}\right]$ induced by the norm map becomes the zero map if $m$ is sufficiently large. We take the minimal $m$ satisfying the above property, and put $K_{[1]}=K_{m}$. We define inductively $K_{[n]}$ by $K_{[n]}=\left(K_{[n-1]}\right)_{[1]}$ where we applied the above definition to $K_{[n-1]}$ instead of $K$. For $n>0$, we put

$$
\mathcal{S}_{[n]}(K)=\mathcal{S}\left(K_{[n]}\right)
$$

and define $\mathcal{N}_{[n]}(K)$ to be the set consisting of all squarefree products of primes in $\mathcal{S}_{[n]}(K)$. We consider $\mathcal{N}_{[\epsilon(\mathfrak{n})]}(K)$.

We assume $\mathfrak{n} \neq 1$ and $\mathfrak{n} \mathfrak{l} \in \mathcal{N}_{[\epsilon(\mathfrak{n})]}(K)$. By the Chebotarev density theorem, we can take $\mathfrak{l}^{\prime} \in \mathcal{S}\left(K_{[\epsilon(\mathfrak{n})]}(\mathfrak{n})\right)$ such that $\mathfrak{l}_{K_{[\epsilon(\mathfrak{n})]}^{\prime}}^{\prime}$ and $\mathfrak{l}_{K_{[\epsilon(\mathfrak{n})]}}$ yield the same class in $A_{K_{[\epsilon(\mathfrak{n})]}}^{\chi}$. By Lemma 3.1 for $K_{[\epsilon(\mathfrak{n})]}$, there is a unique element $b_{[\epsilon(\mathfrak{n})]} \in\left(K_{[\epsilon(\mathfrak{n})]}^{\times} \otimes \mathbf{Z}_{p}\right)^{\chi}$ such that $\operatorname{div}\left(b_{[\epsilon(\mathfrak{n})]}\right)=$ $\left(\mathfrak{l}_{K_{[\epsilon(\mathfrak{n})]}^{\prime}}-\mathfrak{l}_{K_{[\epsilon(\mathfrak{n})]}}\right)^{\chi}$. Put $b=N_{K_{[\epsilon(\mathfrak{n})]} / K_{[1]}}\left(b_{[\epsilon(\mathfrak{n})]}\right)$. Again, using induction on $\epsilon(\mathfrak{n})$, we define

$$
\left(\widetilde{\kappa}_{\mathfrak{n}, \mathfrak{l}}^{K_{[1]}}\right)^{\prime}=\tilde{\kappa}_{\mathfrak{n}, \mathfrak{l}^{\prime}}^{K_{[1]}}-\tilde{\delta}_{\mathfrak{n}}^{K_{[1]}} b-\sum_{\mathfrak{r} \mid \mathfrak{n}}\left(\left(\widetilde{\kappa}_{\frac{\mathfrak{n}}{\mathfrak{r}}, \mathfrak{r}}^{K_{[1]}}\right)^{\prime} \otimes \phi_{\mathfrak{r}}^{K_{[1]}}(b)\right) \in\left(K_{[1]}^{\times} / p^{N}\right)^{\chi} \otimes \mathcal{G}_{\mathfrak{n}},
$$

and $\tilde{\kappa}_{\mathfrak{n}, \mathfrak{l}}=N_{K_{[1]} / K}\left(\left(\tilde{\kappa}_{\mathfrak{n}, \mathfrak{l}}^{K_{[1]}}\right)^{\prime}\right) \in\left(K^{\times} / p^{N}\right)^{\chi} \otimes \mathcal{G}_{\mathfrak{n}}$. Then we can prove that this element $\tilde{\kappa}_{\mathfrak{n}, \mathfrak{l}}$ does not depend on the choice of $\mathfrak{l}^{\prime}$, and satisfies the properties in Proposition 4.2. This definition looks similar to the first definition, but this method is useful when we study more general Galois representations (see $[\mathbf{1 1}]$ ).

4.3. The following lemma is useful when we choose $\mathfrak{l}^{\prime}$ in the definition of $\tilde{\kappa}_{\mathfrak{n}, \mathfrak{l}}$ in the previous subsection.

LemMA 4.5. Assume $\mathfrak{n}=\mathfrak{r}_{1} \cdot \ldots \cdot \mathfrak{r}_{m} \in \mathcal{N}(K)$ and $\mathfrak{l} \in \mathcal{S}(K)$ is prime to $\mathfrak{n}$. Suppose for each $i=1, \ldots, m, \sigma_{i} \in O_{\chi} / p^{N}[\Gamma(K / k)] \otimes G_{\mathfrak{r}_{i}}$ is given. Then there are infinitely many $\mathfrak{l}^{\prime} \in \mathcal{S}(K(\mathfrak{n}))$ which satisfy the following properties.

(i) The class $\left[\mathfrak{l}_{K}^{\prime}\right]^{\chi}$ in $A_{K}^{\chi}$ coincides with the class $\left[\mathfrak{l}_{K}\right]^{\chi}$.

(ii) For the element $z \in\left(K^{\times} \otimes \mathbf{Z}_{p}\right)^{\chi} \operatorname{such}$ that $\operatorname{div}(z)=\left(\mathfrak{l}_{K}^{\prime}-\mathfrak{l}_{K}\right)^{\chi}, \phi_{\mathfrak{r}_{i}}(z)=\sigma_{i}$ holds for each $i=1, \ldots, m$. 
Proof. Let $K\{\mathfrak{n}\}$ be the maximal abelian $p$-extension of $K$ which is unramified outside $\mathfrak{n}$. For a prime $v$ of $K$, we define $U_{K_{v}}=O_{K_{v}}^{\times}$and $U_{K_{v}}^{1}=1+m_{v} O_{K_{v}}$ as usual where $m_{v}$ is the maximal ideal of $O_{K_{v}}$. The residue field of $v$ is denoted by $\kappa(v)$. By class field theory, we have an isomorphism

$$
\frac{\prod_{v \mid \mathfrak{n}} K_{v}^{\times} / U_{K_{v}}^{1} \times \bigoplus_{v \nmid \mathfrak{n}} K_{v}^{\times} / U_{K_{v}}}{\text { the image of } K^{\times}} \otimes \mathbf{Z}_{p} \stackrel{\simeq}{\longrightarrow} \operatorname{Gal}(K\{\mathfrak{n}\} / K)
$$

which yields an exact sequence

$$
0 \longrightarrow\left(\bigoplus_{v \mid \mathfrak{n}} \kappa(v)^{\times} \otimes \mathbf{Z}_{p}\right)^{\chi} \longrightarrow \operatorname{Gal}(K\{\mathfrak{n}\} / K)^{\chi} \longrightarrow A_{K}^{\chi} \longrightarrow 0
$$

where the injectivity of the second arrow follows from $\left(O_{K}^{\times} \otimes \mathbf{Z}_{p}\right)^{\chi}=0$. We denote by $K\{\mathfrak{n}\}^{\chi}$ the intermediate field of $K\{\mathfrak{n}\} / K$ such that $\operatorname{Gal}\left(K\{\mathfrak{n}\}^{\chi} / K\right)=\operatorname{Gal}(K\{\mathfrak{n}\} / K)^{\chi}$.

By the similar method as we did when we defined $\phi_{\mathfrak{l}}$ in $\S 2.3$, we identify $\left(\bigoplus_{v \mid \mathfrak{n}} \kappa(v)^{\times} \otimes\right.$ $\left.\mathbf{Z}_{p}\right)^{\chi}$ with $O_{\chi}[\Gamma(K / k)] \otimes G_{\mathfrak{r}_{1}} \oplus \ldots \oplus O_{\chi}[\Gamma(K / k)] \otimes G_{\mathfrak{r}_{m}}$. We take $\sigma_{\chi} \in\left(\bigoplus_{v \mid \mathfrak{n}} \kappa(v)^{\times} \otimes \mathbf{Z}_{p}\right)^{\chi}$ such that $\sigma_{\chi} \bmod p^{N}$ is $\left(\sigma_{1}, \ldots, \sigma_{m}\right)$, and regard $\sigma_{\chi}$ as an element of $\operatorname{Gal}\left(K\{\mathfrak{n}\}^{\chi} / K\right)$. Let $\left(\mathfrak{l}_{K}, K\{\mathfrak{n}\}^{\chi} / K\right) \in \operatorname{Gal}\left(K\{\mathfrak{n}\}^{\chi} / K\right)$ be the Artin symbol (the Frobenius of $\left.\mathfrak{l}_{K}\right)$. Note that $\Delta(K / k)$ acts trivially on $\operatorname{Gal}(K(\mathfrak{n}) / K)$, so $K\{\mathfrak{n}\}^{\chi} \cap K(\mathfrak{n})=K$. Put $L=K\{\mathfrak{n}\}^{\chi} K(\mathfrak{n})$ which is a subfield of $K\{\mathfrak{n}\}$. We take $\tau \in \operatorname{Gal}(L / K)$ whose image in $\operatorname{Gal}\left(K\{\mathfrak{n}\}^{\chi} / K\right)$ is $\sigma_{\chi}^{-1}\left(\mathfrak{l}_{K}, K\{\mathfrak{n}\}^{\chi} / K\right)$ and whose image in $\operatorname{Gal}(K(\mathfrak{n}) / K)$ is the identity map.

Let $\mathcal{U}$ be as in the proof of Lemma 2.1. Considering the action of $\operatorname{Gal}\left(K_{0}\left(\mu_{p}\right) / k\right)$, we have $K\left(\mu_{p^{n+1}}, \mathcal{U}^{1 / p^{n}}\right) \cap L=K$. Hence by the Chebotarev density theorem there exist infinitely many $\mathfrak{l}^{\prime} \in \mathcal{S}(K)$ such that $\left(\mathfrak{l}_{K}^{\prime}, L / K\right)=\tau$ in $\operatorname{Gal}(L / K)$ where $\left(\mathfrak{l}_{K}^{\prime}, L / K\right)$ is the Frobenius of $\mathfrak{l}_{K}^{\prime}$ in $\operatorname{Gal}(L / K)$.

Since the image of $\left(\mathfrak{l}_{K}^{\prime}, L / K\right)$ in $\operatorname{Gal}(K(\mathfrak{n}) / K)$ is the identity, $\mathfrak{l}^{\prime}$ is in $\mathcal{S}(K(\mathfrak{n}))$. Let $\Pi_{\mathfrak{l}^{\prime}}$ be the idele whose $\mathfrak{l}_{K}^{\prime}$-component is a prime element of $\mathfrak{l}_{K}^{\prime}$ and whose other components are trivial. Let $\Pi_{\mathfrak{l}, \sigma_{\chi}}$ denote the idele whose $\left(\Pi_{v \mid \mathfrak{n}} K_{v}^{\times} \otimes \mathbf{Z}_{p}\right)^{\chi}$-component is $\tilde{\sigma}_{\chi}^{-1} \in\left(\Pi_{v \mid \mathfrak{n}} K_{v}^{\times} \otimes \mathbf{Z}_{p}\right)^{\chi}$ which is a lifting of $\sigma_{\chi}^{-1} \in\left(\Pi_{v \mid \mathfrak{n}} U_{K_{v}} / U_{K_{v}}^{1} \otimes \mathbf{Z}_{p}\right)^{\chi}$, and whose $\mathfrak{l}_{K}$-component is a prime element of $\mathfrak{l}_{K}$ and whose other components are trivial. By definition, $\Pi_{\mathfrak{l}^{\prime}}$ and $\Pi_{\mathfrak{l}, \sigma_{\chi}}$ have the same class in

$$
\left(\frac{\prod_{v \mid \mathfrak{n}} K_{v}^{\times} / U_{K_{v}}^{1} \times \bigoplus_{v \nmid \mathfrak{n}} K_{v}^{\times} / U_{K_{v}}}{\text { the image of } K^{\times}} \otimes \mathbf{Z}_{p}\right)^{\chi}=\operatorname{Gal}\left(K\{\mathfrak{n}\}^{\chi} / K\right) .
$$

Hence there is an element $z \in\left(K^{\times} \otimes \mathbf{Z}_{p}\right)^{\chi}$ such that $\Pi_{\mathfrak{l}^{\prime}}=z \Pi_{\mathfrak{l}, \sigma_{\chi}}$ in $\left(\left(\prod_{v \mid \mathfrak{n}} K_{v}^{\times} / U_{K_{v}}^{1} \times\right.\right.$ $\left.\left.\bigoplus_{v \nmid \mathfrak{n}} K_{v}^{\times} / U_{K_{v}}\right) \otimes \mathbf{Z}_{p}\right)^{\chi}$. Therefore, the class $\left[\mathfrak{l}_{K}^{\prime}\right]^{\chi}$ in $A_{K}^{\chi}$ coincides with the class $\left[\mathfrak{l}_{K}\right]^{\chi}$, and $\operatorname{div}(z)=\left(\mathfrak{l}_{K}^{\prime}-\mathfrak{l}_{K}\right)^{\chi}$. Furthermore, $\phi_{\mathfrak{r}_{i}}(z)=\sigma_{i}$ for all $i=1, \ldots, m$.

REMARK 4.6. In the definition of $\tilde{\kappa}_{\mathfrak{n}, \mathfrak{r}}^{\prime}$ in $\S 4.2$, using Lemma 4.5, we can take $\mathfrak{l}^{\prime} \in \mathcal{S}(K(\mathfrak{n}))$ and $b \in\left(K^{\times} \otimes \mathbf{Z}_{p}\right)^{\chi}$ such that $\operatorname{div}(b)=\left(\mathfrak{l}_{K}^{\prime}-\mathfrak{l}_{K}\right)^{\chi}$ and $\phi_{\mathfrak{r}}^{K}(b)=0$ in $O_{\chi} / p^{N+\epsilon(\mathfrak{n}) c}[\Gamma(K / k)] \otimes$ $G_{\mathfrak{n}}$ for all $\mathfrak{r}$ dividing $\mathfrak{n}$. Then we have $\tilde{\kappa}_{\mathfrak{n}, \mathfrak{l}}^{\prime}=\tilde{\kappa}_{\mathfrak{n}, \mathfrak{l}^{\prime}}^{(N+\epsilon(\mathfrak{n}) c)}-\tilde{\delta}_{\mathfrak{n}}^{(N+\epsilon(\mathfrak{n}) c)} b$, which implies that

$$
\tilde{\kappa}_{\mathfrak{n}, \mathfrak{l}}=\tilde{\kappa}_{\mathfrak{n}, \mathfrak{l}^{\prime}}^{(N+\epsilon(\mathfrak{n}) c)}-\tilde{\delta}_{\mathfrak{n}}^{(N+\epsilon(\mathfrak{n}) c)} b \bmod p^{N} .
$$

This fact will be used later.

\section{Kolyvagin systems of Gauss sum type II}

In this section, we study $\phi_{\mathfrak{r}}\left(\tilde{\kappa}_{\mathfrak{n}, \mathfrak{l}}\right)$ for $\mathfrak{r}$ dividing $\mathfrak{n} \mathfrak{l}$. We use the same notation $K, \chi, g_{\mathfrak{l}}^{\chi}, \tilde{\kappa}_{\mathfrak{n}, \mathfrak{l}}$, etc as in the previous section. 
5.1. Suppose that $\mathfrak{l} \in \mathcal{S}(K)$. We consider the homomorphism

$$
\phi_{\mathfrak{l}}:\left(K^{\times} / p^{N}\right)^{\chi} \longrightarrow O_{\chi} / p^{N}[\Gamma(K / k)] \otimes G_{\mathfrak{l}}
$$

defined in $\S 2.3$.

Proposition 5.1. $\quad \phi_{\mathfrak{l}}\left(g_{\mathfrak{l}}^{K}\right)=-\delta_{\mathfrak{l}} \otimes \sigma_{\mathfrak{l}}=-\tilde{\delta}_{\mathfrak{l}}$.

When $k=\mathbf{Q}$, this proposition and the next corollary correspond to Theorem 2.4 in Rubin [16] where it was proved by using the explicit form of Gauss sums. We do not know the explicit form of our $g_{\mathfrak{l}}^{K}$, so we prove this proposition by a completely different method which can be applied to general $k$. This proposition can be formulated in a simple form as above, because the homomorphism $\phi_{\mathfrak{l}}$ is defined not only on the l-units but defined on the whole $K^{\times}$by using the reciprocity map.

Proof of Proposition 5.1. Put $L=K(\mathfrak{l})$. As in the proof of Proposition 3.2, using the Chebotarev density theorem, we can take $\mathfrak{l}^{\prime} \in \mathcal{S}(L)$ such that the class $\left[\mathfrak{l}_{L}^{\prime}\right]^{\chi}$ in $A_{L}^{\chi}$ coincides with the class $\left[\mathfrak{l}_{L}\right]^{\chi}$ in $A_{L}^{\chi}$ where $\mathfrak{l}_{L}$ is the unique prime of $L$ above $\mathfrak{l}_{K}$. We take $a \in\left(L^{\times} \otimes \mathbf{Z}_{p}\right)^{\chi}$ such that $\operatorname{div}(a)=\left(\mathfrak{l}_{L}^{\prime}-\mathfrak{l}_{L}\right)^{\chi}$.

Let $c_{L / K}: O_{\chi}[\Gamma(L / k)] \longrightarrow O_{\chi}[\Gamma(K / k)]$ be the natural restriction map. By Lemma 2.4, we have $c_{L / K}\left(\theta_{L}^{\chi}\right)=\left(1-\varphi_{\mathfrak{l}}^{-1}\right) \theta_{K}^{\chi}=0$ because $\mathfrak{l} \in \mathcal{S}(K)$ implies $\varphi_{\mathfrak{l}}=1$. Hence $\sigma_{\mathfrak{l}}-1$ divides $\theta_{L}^{\chi}$, and we can write

$$
\theta_{L}^{\chi}=\alpha\left(\sigma_{\mathfrak{l}}-1\right)+\beta\left(\sigma_{\mathfrak{l}}-1\right)^{2}
$$

for some $\alpha \in O_{\chi}[\Gamma(K / k)]$ and $\beta \in O_{\chi}[\Gamma(L / k)]$. We have $\alpha \equiv-\delta_{\mathfrak{l}} \quad\left(\bmod p^{N}\right)$ by $(3.3)$ in $\S 3.3$ (Lemma 4.4 in $[\mathbf{1 0}]$ ). Since $\mathfrak{l}_{L}$ is totally ramified in $L / K$, we have $\left(\sigma_{\mathfrak{l}}-1\right) \mathfrak{l}_{L}=0$. It follows from $\sigma_{\mathfrak{l}}-1 \mid \theta_{L}^{\chi}$ that $\theta_{L}^{\chi}\left(\mathfrak{l}_{L}\right)^{\chi}=0$ in $\left(\operatorname{Div}_{L} \otimes \mathbf{Z}_{p}\right)^{\chi}$. Therefore, we have

$$
\operatorname{div}\left(g_{\mathfrak{l}^{\prime}}^{L}\right)=\theta_{L}^{\chi}\left(\mathfrak{l}_{L}^{\prime}\right)^{\chi}=\theta_{L}^{\chi}\left(\mathfrak{l}_{L}^{\prime}-\mathfrak{l}_{L}\right)^{\chi}=\operatorname{div}\left(a^{\theta_{L}^{\chi}}\right)
$$

which implies $g_{\mathrm{r}^{\prime}}^{L}=a^{\theta_{L}^{\chi}}$ by Lemma 3.1.

Put $z=a^{\alpha+\beta\left(\sigma_{\mathfrak{l}}-1\right)} \in\left(L^{\times} \otimes \mathbf{Z}_{p}\right)^{\chi}$. Using the congruence relation (Proposition 3.2), we compute

$$
\begin{aligned}
\left(\sigma_{\mathfrak{l}}-1\right) z & =a^{\theta_{L}^{\chi}}=g_{\mathfrak{l}^{\prime}}^{L} \equiv\left(g_{\mathfrak{l}^{\prime}}^{K}\right)^{\frac{1-N(\mathfrak{l})-1}{p^{n} \mathfrak{l}}} \\
& \equiv\left(g_{\mathfrak{l}^{\prime}}^{K}\right)^{\frac{N(\mathfrak{l})-1}{p^{n} \mathfrak{l}}} \equiv\left(g_{\mathfrak{l}^{\prime}}^{K}\right)^{-\frac{1-N(\mathfrak{l})}{p^{n} \mathfrak{l}}} \quad\left(\bmod \rho_{\mathfrak{l}}\right)
\end{aligned}
$$

for any prime $\rho_{\mathfrak{l}}$ of $L$ above $\mathfrak{l}$.

We denote by

$$
\operatorname{div}_{\mathfrak{l}_{L}}:\left(L^{\times} \otimes \mathbf{Z}_{p}\right)^{\chi} \longrightarrow\left(\bigoplus_{\rho \mid \mathfrak{l}} \mathbf{Z}_{p}\right)^{\chi} \stackrel{\simeq}{\longrightarrow} O_{\chi}[\Gamma(K / k)]
$$

the homomorphism induced by $x \mapsto \Sigma_{\rho \mid \mathfrak{r}} \operatorname{ord}_{\rho}(x) \rho$ where $\rho$ runs through all primes of $L$ above $\mathfrak{l}$, and the second isomorphism is $\vartheta\left(\mathfrak{l}_{L}\right)^{\chi} \mapsto \vartheta$ for $\vartheta \in O_{\chi}[\Gamma(K / k)]$. It follows from Lemma 2.2 that

$$
\begin{aligned}
\phi_{\mathfrak{l}}\left(g_{\mathfrak{l}^{\prime}}^{K}\right) & =-\operatorname{div}_{\mathfrak{l}_{L}}(z) \otimes \sigma_{\mathfrak{l}} \bmod p^{N} \\
& =-\left(\alpha+\beta\left(\sigma_{\mathfrak{l}}-1\right)\right) \operatorname{div}_{\mathfrak{l}_{L}}(a) \otimes \sigma_{\mathfrak{l}} \bmod p^{N} \\
& =-\alpha \operatorname{div}_{\mathfrak{l}_{L}}(a) \otimes \sigma_{\mathfrak{l}} \bmod p^{N}=\alpha \otimes \sigma_{\mathfrak{l}} \bmod p^{N} \\
& =-\delta_{\mathfrak{l}} \otimes \sigma_{\mathfrak{l}} .
\end{aligned}
$$

Put $b=N_{L / K}(a) \in\left(K^{\times} \otimes \mathbf{Z}_{p}\right)^{\chi}$. By the definition of $a$, we have $\operatorname{div}(b)=\left(\mathfrak{l}_{K}^{\prime}-\mathfrak{l}_{K}\right)^{\chi}$. Since $\operatorname{div}\left(g_{\mathfrak{l}}^{K}\right)=\operatorname{div}\left(g_{\mathfrak{l}^{\prime}}^{K} / b^{\theta_{K}^{\chi}}\right)$, by Lemma 3.1 we obtain $g_{\mathfrak{l}}^{K}=g_{\mathfrak{l}^{\prime}}^{K} / b_{K}^{\theta_{K}^{\chi}}$. Since $b$ is a norm from $L$, by 
the remark in the end of $\S 2.3$ (by local class field theory), we know $\phi_{\mathfrak{l}}(b)=0$. Therefore, we finally have

$$
\phi_{\mathfrak{l}}\left(g_{\mathfrak{l}}^{K}\right)=\phi_{\mathfrak{l}}\left(g_{\mathfrak{l}^{\prime}}^{K}\right)=-\delta_{\mathfrak{l}} \otimes \sigma_{\mathfrak{l}}=-\tilde{\delta}_{\mathfrak{l}}
$$

Next, let us consider a map

$$
\left(K^{\times} / p^{N}\right)^{\chi} \otimes \mathcal{G}_{\mathfrak{n}} \longrightarrow O_{\chi} / p^{N}[\Gamma(K / k)] \otimes \mathcal{G}_{\mathfrak{n} \mathfrak{l}}
$$

which is obtained from $\phi_{\mathfrak{l}}:\left(K^{\times} / p^{N}\right)^{\chi} \longrightarrow O_{\chi} / p^{N}[\Gamma(K / k)] \otimes \mathcal{G}_{\mathfrak{l}}$ by tensoring $\mathcal{G}_{\mathfrak{n}}$, and which we also denote by $\phi_{\mathrm{l}}$.

COROLlary 5.2. We assume that $\mathfrak{l} \in \mathcal{S}(K(\mathfrak{n}))$. Then we have $\phi_{\mathfrak{l}}\left(\tilde{\kappa}_{\mathfrak{n}, \mathfrak{l}}\right)=-\tilde{\delta}_{\mathfrak{n} \mathfrak{l}}$.

Proof. We denote $\delta_{\mathfrak{l}}, \phi_{\mathfrak{l}}$ for $K(\mathfrak{n})$ by $\delta_{\mathfrak{l}}^{K(\mathfrak{n})}, \phi_{\mathfrak{l}}^{K(\mathfrak{n})}$. We apply Proposition 5.1 to $K(\mathfrak{n})$ to get

$$
\phi_{\mathfrak{l}}^{K(\mathfrak{n})}\left(g_{\mathfrak{l}}^{K(\mathfrak{n})}\right)=-\delta_{\mathfrak{l}}^{K(\mathfrak{n})} \otimes \sigma_{\mathfrak{l}} \in O_{\chi} / p^{N}[\Gamma(K(\mathfrak{n}) / k)] \otimes G_{\mathfrak{l}}=O_{\chi} / p^{N}\left[\Gamma(K / k) \times G_{\mathfrak{n}}\right] \otimes G_{\mathfrak{l}} .
$$

Consider the commutative diagram

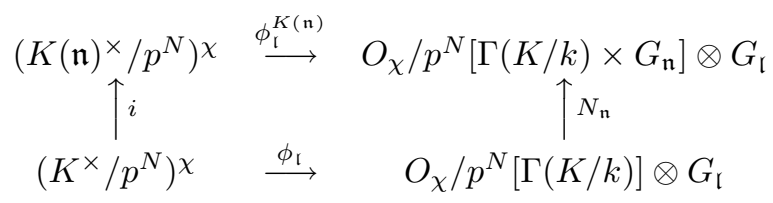

where $i$ is the natural inclusion map, and $N_{\mathfrak{n}}$ is the multiplication by $N_{\mathfrak{n}}=N_{G_{\mathfrak{n}}}=\Sigma_{\sigma \in G_{\mathfrak{n}}} \sigma$. Since $i\left(\kappa_{\mathfrak{n}, \mathfrak{l}}\right)=D_{\mathfrak{n}} g_{\mathfrak{l}}^{K(\mathfrak{n})}$ and $D_{\mathfrak{n}} \delta_{\mathfrak{l}}^{K(\mathfrak{n})} \otimes \sigma_{\mathfrak{l}}=N_{\mathfrak{n}} \delta_{\mathfrak{n} \mathfrak{l}} \otimes \sigma_{\mathfrak{l}}$, we have $\phi_{\mathfrak{l}}\left(\kappa_{\mathfrak{n}, \mathfrak{l}}\right)=-\delta_{\mathfrak{n} \mathfrak{l}} \otimes \sigma_{\mathfrak{l}}$ because $N_{\mathfrak{n}}$ is injective. Thus, we get $\phi_{\mathfrak{l}}\left(\widetilde{\kappa}_{\mathfrak{n}, \mathfrak{l}}\right)=-\tilde{\delta}_{\mathfrak{n} \mathfrak{l}}$.

5.2. The homomorphism $\phi_{\mathfrak{r}}:\left(K^{\times} / p^{N}\right)^{\chi} \longrightarrow O_{\chi} / p^{N}[\Gamma(K / k)] \otimes \mathcal{G}_{\mathfrak{r}}$ induces

$$
\left(K^{\times} / p^{N}\right)^{\chi} \otimes \mathcal{G}_{\mathfrak{n}} \longrightarrow O_{\chi} / p^{N}[\Gamma(K / k)] \otimes \mathcal{G}_{\mathfrak{n}} \otimes \mathcal{G}_{\mathfrak{r}}
$$

which we also denote by $\phi_{\mathfrak{r}}$. In [12], for completely general $\mathfrak{n}$, Mazur and Rubin computed $\phi_{\mathfrak{r}}\left(\tilde{\kappa}_{\mathfrak{n}}\right)$ for each $\mathfrak{r} \mid \mathfrak{n}$. In this paper, we consider the following special case.

Suppose that $\mathfrak{n} \in \mathcal{N}(K)$. We call $\mathfrak{n}$ well-ordered if $\mathfrak{n}$ has factorization $\mathfrak{n}=\mathfrak{r}_{1} \cdot \ldots \cdot \mathfrak{r}_{m}$ such that $\mathfrak{r}_{i+1} \in \mathcal{S}\left(K\left(\mathfrak{r}_{1} \cdot \ldots \cdot \mathfrak{r}_{i}\right)\right)$ for all $i=1, \ldots, m-1$. The next lemma follows from Theorem A4 in Mazur and Rubin [12].

Lemma 5.3. (Mazur and Rubin) Assume that $\mathfrak{l} \in \mathcal{S}(K(\mathfrak{n}))$ and $\mathfrak{n}$ is well-ordered. Then, for each $\mathfrak{r} \mid \mathfrak{n}$, we have $\phi_{\mathfrak{r}}\left(\tilde{\kappa}_{\mathfrak{n}, \mathfrak{l}}\right)=0$.

Proof. Since we are in a special case, this lemma can be proved simply. Suppose that $\mathfrak{n}=$ $\mathfrak{r}_{1} \cdot \ldots \cdot \mathfrak{r}_{m}$, and $\mathfrak{r}_{i+1} \in \mathcal{S}\left(K\left(\mathfrak{r}_{1} \cdot \ldots \cdot \mathfrak{r}_{i}\right)\right)$ for all $i=1, \ldots, m-1$, and suppose that $\mathfrak{r}=\mathfrak{r}_{j}$ for some

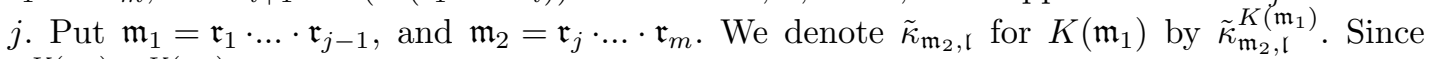
$\phi_{\mathfrak{r}}^{K\left(\mathfrak{m}_{1}\right)}\left(\tilde{\kappa}_{\mathfrak{m}_{2}, \mathfrak{l}}^{K\left(\mathfrak{m}_{1}\right)}\right)=0$ implies $\phi_{\mathfrak{r}}\left(\tilde{\kappa}_{\mathfrak{n}, \mathfrak{l}}\right)=0$, to prove Lemma 5.3 we may assume $\mathfrak{r}=\mathfrak{r}_{1}$.

For a prime $v$ of $K$ above $\mathfrak{r}$, we will prove

$$
\phi_{K_{v}}\left(\kappa_{\mathfrak{n}, \mathfrak{l}}\right)=0
$$

where $\phi_{K_{v}}: K_{v}^{\times} / p^{N} \longrightarrow G_{\mathfrak{r}} \otimes \mathbf{Z} / p^{N}$ is the reciprocity map of $K_{v}$. Let $K_{v}^{\times} / p^{N}=V_{1} \oplus V_{2}$ be the decomposition in Lemma 2.3. Let $v^{\prime}$ be the prime of $K(\mathfrak{r})$ above $v$. Since $v$ is totally ramified in $K(\mathfrak{r}) / K$, the natural map $K_{v}^{\times} / p^{N} \longrightarrow K(\mathfrak{r})_{v^{\prime}}^{\times} / p^{N}$ is injective on $V_{1}$. It follows from this fact 
and $V_{2}=\operatorname{Ker} \phi_{K_{v}}$ that it is enough to show that the image of $\kappa_{\mathfrak{n}, \mathfrak{l}}$ in $K(\mathfrak{r})_{v^{\prime}}^{\times} / p^{N}$ vanishes, in order to get $\phi_{K_{v}}\left(\kappa_{\mathfrak{n}, \mathfrak{l}}\right)=0$.

We note that the image of $\kappa_{\mathfrak{n}, \mathfrak{l}}$ in $K(\mathfrak{r})^{\times} / p^{N}$ is $D_{\mathfrak{r}} \kappa_{\frac{\mathfrak{n}}{\mathfrak{r}} \mathfrak{l}}^{K(\mathfrak{r})}$. Let $U_{K(\mathfrak{r})_{v^{\prime}}}$ be the unit group of $K(\mathfrak{r})_{v^{\prime}}$. The image of $D_{\mathfrak{r}} \kappa_{\frac{\mathfrak{n}}{\mathfrak{r}}, \mathfrak{l}}^{K(\mathfrak{r})}$ in $K(\mathfrak{r})_{v^{\prime}}^{\times} / p^{N}$ is in $U_{K(\mathfrak{r})_{v^{\prime}}} / p^{N}$. Since $G_{\mathfrak{r}}$ acts on $U_{K(\mathfrak{r})_{v^{\prime}}} / p^{N}$ trivially, $D_{\mathfrak{r}}$ acts on $U_{K(\mathfrak{r})_{v^{\prime}}} / p^{N}$ as $\frac{p^{n_{\mathfrak{r}}}\left(p^{n_{\mathfrak{r}}}-1\right)}{2}=0$. Therefore, the image of $D_{\mathfrak{r}} \kappa_{\frac{\mathfrak{n}}{\mathfrak{r}}, \mathfrak{l}}^{K(\mathfrak{r})}$ in $U_{K(\mathfrak{r})_{v^{\prime}}} / p^{N}$ is zero. Thus, we get $\phi_{K_{v}}\left(\kappa_{\mathfrak{n}, \mathfrak{l}}\right)=0$, which implies $\phi_{\mathfrak{r}}\left(\tilde{\kappa}_{\mathfrak{n}, \mathfrak{l}}\right)=0$.

Recall that we defined in $\S 4 \tilde{\kappa}_{\mathfrak{n}, \mathfrak{l}} \in\left(K^{\times} / p^{N}\right)^{\chi} \otimes \mathcal{G}_{\mathfrak{n}}$ for $\mathfrak{n}$ and $\mathfrak{l}$ such that $\mathfrak{n} \mathfrak{l} \in \mathcal{N}_{[\epsilon(\mathfrak{n})]}(K)$.

Proposition 5.4. Assume that $\mathfrak{n} \mathfrak{l} \in \mathcal{N}_{[\epsilon(\mathfrak{n})]}(K)$ and $\mathfrak{n}$ is well-ordered. Then, for each $\mathfrak{r} \mid \mathfrak{n}$, we have $\phi_{\mathfrak{r}}\left(\tilde{\kappa}_{\mathfrak{n}, \mathfrak{l}}\right)=0$.

Proof. As we remarked in Remark 4.6, in the definition of $\tilde{\kappa}_{\mathfrak{n}, \mathfrak{r}}^{\prime}$ in $\S 4.2$, using Lemma 4.5 we can take $b \in\left(K^{\times} \otimes \mathbf{Z}_{p}\right)^{\chi}$ such that $\operatorname{div}(b)=\left(\mathfrak{l}_{K}^{\prime}-\mathfrak{l}_{K}\right)^{\chi}$ and $\phi_{\mathfrak{r}}(b)=0$ in $O_{\chi} / p^{N+\epsilon(\mathfrak{n}) c}[\Gamma(K / k)] \otimes$ $G_{\mathfrak{n}}$ for all $\mathfrak{r}$ dividing $\mathfrak{n}$. Then

$$
\tilde{\kappa}_{\mathfrak{n}, \mathfrak{l}}=\tilde{\kappa}_{\mathfrak{n}, \mathfrak{l}^{\prime}}^{(N+\epsilon(\mathfrak{n}) c)}-\tilde{\delta}_{\mathfrak{n}}^{(N+\epsilon(\mathfrak{n}) c)} b \quad\left(\bmod p^{N}\right) .
$$

Therefore, by Lemma 5.3 and $\phi_{\mathfrak{r}}(b)=0$, we obtain

$$
\phi_{\mathfrak{r}}\left(\tilde{\kappa}_{\mathfrak{n}, \mathfrak{l}}\right)=\phi_{\mathfrak{r}}\left(\tilde{\kappa}_{\mathfrak{n}, \mathfrak{l}^{\prime}}\right)-\tilde{\delta}_{\mathfrak{n}} \phi_{\mathfrak{r}}(b)=0 .
$$

We next consider

$$
\phi_{\mathfrak{l}}:\left(K^{\times} / p^{N}\right)^{\chi} \otimes \mathcal{G}_{\mathfrak{n}} \longrightarrow O_{\chi} / p^{N}[\Gamma(K / k)] \otimes \mathcal{G}_{\mathfrak{n} \mathfrak{l}}
$$

which is induced by $\phi_{\mathfrak{l}}:\left(K^{\times} / p^{N}\right)^{\chi} \longrightarrow O_{\chi} / p^{N}[\Gamma(K / k)] \otimes \mathcal{G}_{\mathfrak{l}}$.

Proposition 5.5. Assume that $\mathfrak{n} \mathfrak{l} \in \mathcal{N}_{[\epsilon(\mathfrak{n})+1]}(K)$ and $\mathfrak{n} \mathfrak{l}$ is well-ordered. Then we have $\phi_{\mathfrak{l}}\left(\tilde{\kappa}_{\mathfrak{n}, \mathfrak{l}}\right)=-\tilde{\delta}_{\mathfrak{n} \mathfrak{l}}$.

The assumption that $\mathfrak{n} \mathfrak{l}$ is well-ordered does not mean $\mathfrak{l} \in \mathcal{S}(K(\mathfrak{n}))$, but means that $\mathfrak{n} \mathfrak{l}$ has factorization $\mathfrak{n} \mathfrak{l}=\mathfrak{r}_{1} \cdot \ldots \cdot \mathfrak{r}_{m+1}$ satisfying the property in the definition of the well-orderedness in the beginning of $\S 5.2$ (namely, $\mathfrak{l}=\mathfrak{r}_{i}$ for some $i$ ).

Proof of Proposition 5.5. We take $\mathfrak{l}^{\prime} \in \mathcal{S}_{[\epsilon(\mathfrak{n})+1]}(K(\mathfrak{n} \mathfrak{l}))=\mathcal{S}_{[\epsilon(\mathfrak{n} \mathfrak{l})]}(K(\mathfrak{n} \mathfrak{l}))$ and consider $\tilde{\kappa}_{\mathfrak{n} \mathfrak{l}, \mathfrak{l}^{\prime}}$ As in Remark 4.6, using Lemma 4.5, we can take an auxiliary prime $\mathfrak{l}^{\prime \prime} \in \mathcal{S}_{[\epsilon(\mathfrak{n} \mathfrak{l})]}(K(\mathfrak{n} \mathfrak{l}))$ and $b \in\left(K^{\times} \otimes \mathbf{Z}_{p}\right)^{\chi}$ such that $\operatorname{div}(b)=\left(\mathfrak{l}_{K}^{\prime \prime}-\mathfrak{l}_{K}^{\prime}\right)^{\chi}, \phi_{\mathfrak{r}}(b)=0$ for all $\mathfrak{r}$ dividing $\mathfrak{n}$, and $\phi_{\mathfrak{l}}(b)=1 \otimes \sigma_{\mathfrak{l}}$ which is a generator of $O_{\chi} / p^{N}[\Gamma(K / k)] \otimes \mathcal{G}_{\mathfrak{l}}$. We showed in Proposition 4.2 that $\tilde{\kappa}_{\mathfrak{n} \mathfrak{l}, \mathfrak{l}^{\prime}}$ does not depend on the choice of $\mathfrak{l}^{\prime \prime}$, namely we have

$$
\tilde{\kappa}_{\mathfrak{n} \mathfrak{l}, \mathfrak{l}^{\prime}}=\tilde{\kappa}_{\mathfrak{n} \mathfrak{l}, \mathfrak{l}^{\prime \prime}}-\tilde{\delta}_{\mathfrak{n} \mathfrak{l}} b-\tilde{\kappa}_{\mathfrak{n}, \mathfrak{l}} \otimes \phi_{\mathfrak{l}}(b) .
$$

Therefore, using $\phi_{\mathfrak{l}}(b)=1 \otimes \sigma_{\mathfrak{l}}$, we have

$$
\phi_{\mathfrak{l}}\left(\tilde{\kappa}_{\mathfrak{n} \mathfrak{l}, \mathfrak{l}^{\prime}}\right)=\phi_{\mathfrak{l}}\left(\tilde{\kappa}_{\mathfrak{n} \mathfrak{l}, \mathfrak{l}^{\prime \prime}}\right)-\tilde{\delta}_{\mathfrak{n} \mathfrak{l}} \otimes \sigma_{\mathfrak{l}}-\phi_{\mathfrak{l}}\left(\tilde{\kappa}_{\mathfrak{n}, \mathfrak{l}}\right) \otimes \sigma_{\mathfrak{l}} .
$$

On the other hand, Proposition 5.4 tells us that $\phi_{\mathfrak{l}}\left(\tilde{\kappa}_{\mathfrak{n} \mathfrak{l}, \mathfrak{l}^{\prime}}\right)=\phi_{\mathfrak{l}}\left(\tilde{\kappa}_{\mathfrak{n} \mathfrak{l}, \mathfrak{l}^{\prime \prime}}\right)=0$. Hence we obtain $\phi_{\mathfrak{l}}\left(\tilde{\kappa}_{\mathfrak{n}, \mathfrak{l}}\right)=-\tilde{\delta}_{\mathfrak{n} \mathfrak{l}}$ in $O_{\chi} / p^{N}[\Gamma(K / k)] \otimes \mathcal{G}_{\mathfrak{n} \mathfrak{l}}$. 
5.3. It seems to the author that one can define $\kappa_{\mathfrak{n}, \mathfrak{l}}$ and $\tilde{\kappa}_{\mathfrak{n}, \mathfrak{l}}$ in a more general setting. In this paper, we defined these elements under the assumption $\mathfrak{n} \mathfrak{l} \in \mathcal{N}_{[\epsilon(\mathfrak{n})]}(K)$ (cf. $\S 4.2$ and Remark 4.4). This assumption is used to show that $\tilde{\kappa}_{\mathfrak{n}, \mathfrak{l}}$ is independent of the choice of $\mathfrak{l}^{\prime}$ and $b$ (cf. Proposition 4.2).

Suppose that $\mathfrak{n} \mathfrak{l} \in \mathcal{N}(K)$. Using Lemma 4.5, we can take $\mathfrak{l}^{\prime} \in \mathcal{S}(K(\mathfrak{n}))$ and $b \in\left(K^{\times} \otimes \mathbf{Z}_{p}\right)^{\chi}$ such that $\operatorname{div}(b)=\left(\mathfrak{l}_{K}^{\prime}-\mathfrak{l}_{K}\right)^{\chi}$ and $\phi_{\mathfrak{r}}(b)=0$ for all $\mathfrak{r} \mid \mathfrak{n}$. We write the group law of $\left(K^{\times} \otimes\right.$ $\left.\mathbf{Z} / p^{N}\right)^{\chi} \otimes \mathcal{G}_{\mathfrak{n}}$ additively as before. Can one show that $\tilde{\kappa}_{\mathfrak{n}, \mathfrak{l}^{\prime}}-\tilde{\delta}_{\mathfrak{n}} b$ is independent of the choice of $\mathfrak{l}^{\prime}$, and hence of $b$ (by taking $\mathfrak{l}^{\prime}$ sufficiently close to $\mathfrak{l}$ )? If the answer would be yes, we could define $\tilde{\kappa}_{\mathfrak{n}, \mathfrak{l}}$ as $\tilde{\kappa}_{\mathfrak{n}, \mathfrak{l}^{\prime}}-\tilde{\delta}_{\mathfrak{n}} b$.

Concerning this question, currently the author can only show Proposition A.1 in Appendix, namely he knows the affirmative answer to this question only in the case $\mathfrak{n}$ is a prime.

Another natural question is the following. Suppose that $\tilde{\kappa}_{\mathfrak{n}, \mathfrak{l}}$ is defined. Can one prove $\phi_{\mathfrak{l}}\left(\tilde{\kappa}_{\mathfrak{n}, \mathfrak{l}}\right)=-\tilde{\delta}_{\mathfrak{n} \mathfrak{l}}$ ? In this paper, we proved this property only for $(\mathfrak{n}, \mathfrak{l})$ such that $\mathfrak{l} \in \mathcal{S}(K(\mathfrak{n}))$ (Corollary 5.2), and for $(\mathfrak{n}, \mathfrak{l})$ such that $\mathfrak{n} \mathfrak{l} \in \mathcal{N}_{[\epsilon(\mathfrak{n})+1]}(K)$ and $\mathfrak{n} \mathfrak{l}$ is well-ordered (Proposition $5.5)$.

\section{Elements $x_{\mathfrak{n}, \mathfrak{l}}$}

In this section, we define elements $x_{\mathfrak{n}, \mathfrak{l}} \in\left(K^{\times} \otimes \mathbf{Z} / p^{N}\right)^{\chi}$ and $\tilde{x}_{\mathfrak{n}, \mathfrak{l}} \in\left(K^{\times} / p^{N}\right)^{\chi} \otimes \mathcal{G}_{\mathfrak{n}}$ for $\mathfrak{n} \mathfrak{l} \in$ $\mathcal{N}_{[\epsilon(\mathfrak{n})]}(K)$.

6.1. We write the group law of $\left(K^{\times} / p^{N}\right)^{\chi} \otimes \mathcal{G}_{\mathfrak{n}}$ additively. We assume that $\mathfrak{n} \mathfrak{l} \in \mathcal{N}_{[\epsilon(\mathfrak{n})]}(K)$ and $\mathfrak{n} \mathfrak{r}$ is well-ordered. Suppose that for each prime $\mathfrak{r}$ which divides $\mathfrak{n}$, an element $a_{\mathfrak{r}} \in$ $O_{\chi} / p^{N}[\Gamma(K / k)] \otimes G_{\mathfrak{r}}$ is given (we will give $a_{\mathfrak{r}}$ explicitly later in $\S 9$, see the paragraph before Lemma 9.2). For a divisor $\mathfrak{d}$ of $\mathfrak{n}$, we define $a_{\mathfrak{d}}$ by

$$
a_{\mathfrak{d}}=\otimes_{\mathfrak{r} \mid \mathfrak{d}} a_{\mathfrak{r}} \in O_{\chi} / p^{N}[\Gamma(K / k)] \otimes \mathcal{G}_{\mathfrak{d}}
$$

where for $\mathfrak{d}=\mathfrak{r}_{1} \cdot \ldots \cdot \mathfrak{r}_{m^{\prime}}$ we identify $\left(O_{\chi} / p^{N}[\Gamma(K / k)] \otimes G_{\mathfrak{r}_{1}}\right) \otimes_{O_{\chi} / p^{N}}[\Gamma(K / k)] \cdots \otimes_{O_{\chi} / p^{N}[\Gamma(K / k)]}$ $\left(O_{\chi} / p^{N}[\Gamma(K / k)] \otimes G_{\mathfrak{r}_{m^{\prime}}}\right)$ with $O_{\chi} / p^{N}[\Gamma(K / k)] \otimes \mathcal{G}_{\mathfrak{d}}$. We put $a_{1}=1$. We define $\tilde{x}_{\mathfrak{n}, \mathfrak{r}}$ by

$$
\tilde{x}_{\mathfrak{n}, \mathfrak{l}}=\sum_{\mathfrak{d} \mid \mathfrak{n}} a_{\mathfrak{d}} \otimes \tilde{\kappa}_{\frac{\mathfrak{n}}{\mathfrak{d}}, \mathfrak{l}} \in\left(K^{\times} / p^{N}\right)^{\chi} \otimes \mathcal{G}_{\mathfrak{n}}
$$

where $a_{\mathfrak{d}} \otimes \tilde{\kappa}_{\frac{\mathfrak{n}}{\mathfrak{d}}, \mathfrak{l}} \in\left(O_{\chi} / p^{N}[\Gamma(K / k)] \otimes \mathcal{G}_{\mathfrak{d}}\right) \otimes_{O_{\chi} / p^{N}[\Gamma(K / k)]}\left(K^{\times} / p^{N}\right)^{\chi} \otimes \mathcal{G}_{\frac{\mathfrak{n}}{\mathfrak{d}}}=\left(K^{\times} / p^{N}\right)^{\chi} \otimes \mathcal{G}_{\mathfrak{n}}$, and the sum is taken over all divisors $\mathfrak{d}$ of $\mathfrak{n}$ including 1 . Namely, $\tilde{x}_{\mathfrak{n}, \mathfrak{r}}$ is defined as a sum of $2^{\epsilon(\mathfrak{n})}$ terms.

Proposition 6.1. (0) If $\rho$ is a prime of $K$ which does not divide $\mathfrak{n} \mathfrak{l}$, it is not in the support of $\operatorname{div}\left(\tilde{x}_{\mathfrak{n}, \mathfrak{l}}\right)$.

(1) For each prime $\mathfrak{r}$ dividing $\mathfrak{n}$, we have $\operatorname{div}_{\mathfrak{r}}\left(\tilde{x}_{\mathfrak{n}, \mathfrak{l}}\right)=\phi_{\mathfrak{r}}\left(\tilde{x}_{\frac{\mathfrak{n}}{\mathfrak{r}}, \mathfrak{l}}\right)$.

(2) For each prime $\mathfrak{r}$ dividing $\mathfrak{n}$, we have $\phi_{\mathfrak{r}}\left(\tilde{x}_{\mathfrak{n}, \mathfrak{l}}\right)=a_{\mathfrak{r}} \otimes \phi_{\mathfrak{r}}\left(\tilde{x}_{\frac{\mathfrak{n}}{\mathfrak{r}}, \mathfrak{l}}\right)$.

Proof. The property (0) is an immediate consequence of Proposition $4.2(0)$.

Concerning (1), using Proposition 4.2 (0) and (1), we compute

$$
\operatorname{div}_{\mathfrak{r}}\left(\tilde{x}_{\mathfrak{n}, \mathfrak{l}}\right)=\sum_{\substack{\mathfrak{d}|\mathfrak{n} \\ \mathfrak{r}| \mathfrak{d}}} a_{\mathfrak{d}} \otimes \operatorname{div}_{\mathfrak{r}}\left(\tilde{\kappa}_{\frac{\mathfrak{n}}{\mathfrak{d}}, \mathfrak{l}}\right)+\sum_{\substack{\mathfrak{d} \mid \mathfrak{n} \\ \mathfrak{r} \backslash \mathfrak{d}}} a_{\mathfrak{d}} \otimes \operatorname{div}_{\mathfrak{r}}\left(\tilde{\kappa}_{\frac{\mathfrak{n}}{\mathfrak{d}}, \mathfrak{l}}\right)
$$




$$
\begin{aligned}
& =\sum_{\substack{\mathfrak{d} \mid \mathfrak{n} \\
\mathfrak{r} \chi \mathfrak{d}}} a_{\mathfrak{d}} \otimes \phi_{\mathfrak{r}}\left(\tilde{\kappa}_{\frac{\mathfrak{n}}{\mathfrak{d} \mathfrak{r}}, \mathfrak{l}}\right) \\
& =\sum_{\mathfrak{d} \mid \frac{\mathfrak{n}}{\mathfrak{r}}} a_{\mathfrak{d}} \otimes \phi_{\mathfrak{r}}\left(\tilde{\kappa}_{\frac{\mathfrak{n}}{\mathfrak{d} \mathfrak{r}}, \mathfrak{l}}\right) \\
& =\phi_{\mathfrak{r}}\left(\sum_{\mathfrak{d} \mid \frac{\mathfrak{n}}{\mathfrak{r}}} a_{\mathfrak{d}} \otimes \tilde{\kappa}_{\frac{\mathfrak{n}}{\mathfrak{d} \mathfrak{r}}, \mathfrak{l}}\right)=\phi_{\mathfrak{r}}\left(\tilde{x}_{\frac{\mathfrak{n}}{\mathfrak{r}}, \mathfrak{l}}\right) .
\end{aligned}
$$

We next prove (2). Using Proposition 5.4, we have

$$
\begin{aligned}
\phi_{\mathfrak{r}}\left(\tilde{x}_{\mathfrak{n}, \mathfrak{l}}\right) & =\sum_{\substack{\mathfrak{d}|\mathfrak{n} \\
\mathfrak{r}| \mathfrak{d}}} a_{\mathfrak{d}} \otimes \phi_{\mathfrak{r}}\left(\tilde{\kappa}_{\frac{\mathfrak{n}}{\mathfrak{d}}, \mathfrak{l}}\right)+\sum_{\substack{\mathfrak{d} \mid \mathfrak{n} \\
\mathfrak{r} \chi \mathfrak{d}}} a_{\mathfrak{d}} \otimes \phi_{\mathfrak{r}}\left(\tilde{\kappa}_{\frac{\mathfrak{n}}{\mathfrak{d}}, \mathfrak{l}}\right) \\
& =\sum_{\substack{\mathfrak{d}|\mathfrak{n} \\
\mathfrak{r}| \mathfrak{d}}} a_{\mathfrak{d}} \otimes \phi_{\mathfrak{r}}\left(\tilde{\kappa}_{\frac{\mathfrak{n}}{\mathfrak{d}}, \mathfrak{l}}\right) \\
& =a_{\mathfrak{r}} \otimes\left(\sum_{\mathfrak{d} \mid \frac{\mathfrak{n}}{\mathfrak{r}}} a_{\mathfrak{d}} \otimes \phi_{\mathfrak{r}}\left(\tilde{\kappa}_{\frac{\mathfrak{n}}{\mathfrak{r} \mathfrak{d}}, \mathfrak{l}}\right)\right) \\
& =a_{\mathfrak{r}} \otimes \phi_{\mathfrak{r}}\left(\tilde{x}_{\frac{\mathfrak{n}}{\mathfrak{r}}, \mathfrak{l}}\right) .
\end{aligned}
$$

Thus, we get Proposition 6.1.

Using Lemma 4.5 as in Remark 4.6, we take $\mathfrak{l}^{\prime} \in \mathcal{S}_{[\epsilon(\mathfrak{n})]}(K(\mathfrak{n}))$ and $b \in\left(K^{\times} \otimes \mathbf{Z}_{p}\right)^{\chi}$ such that $\operatorname{div}(b)=\left(\mathfrak{l}_{K}^{\prime}-\mathfrak{l}_{K}\right)^{\chi}$ and $\phi_{\mathfrak{r}}(b)=0$ in $O_{\chi} / p^{N+\epsilon(\mathfrak{n}) c}[\Gamma(K / k)] \otimes G_{\mathfrak{r}}$ for all $\mathfrak{r}$ dividing $\mathfrak{n}$.

\section{LEMMA 6.2 .}

$$
\phi_{\mathfrak{l}^{\prime}}\left(\tilde{x}_{\mathfrak{n}, \mathfrak{l}}\right)=-\sum_{\mathfrak{d} \mid \mathfrak{n}} a_{\mathfrak{d}} \otimes\left(\tilde{\delta}_{\frac{\mathfrak{n}}{\mathfrak{d}} \mathfrak{l}^{\prime}}+\tilde{\delta}_{\frac{\mathfrak{n}}{\mathfrak{d}}} \otimes \phi_{\mathfrak{l}^{\prime}}(b)\right) .
$$

Proof. As in Remark 4.6, we have $\tilde{\kappa}_{\frac{\mathfrak{n}}{\mathfrak{d}}, \mathfrak{l}}=\tilde{\kappa}_{\frac{\mathfrak{n}}{\mathfrak{d}}, \mathfrak{l}^{\prime}}-\tilde{\delta}_{\frac{\mathfrak{n}}{\mathfrak{d}}} b$. Therefore, by the definition of $\tilde{x}_{\mathfrak{n}, \mathfrak{l}}$ we get $\tilde{x}_{\mathfrak{n}, \mathfrak{l}}=\tilde{x}_{\mathfrak{n}, \mathfrak{l}^{\prime}}-\left(\sum_{\mathfrak{d} \mid \mathfrak{n}} a_{\mathfrak{d}} \otimes \tilde{\delta}_{\frac{\mathfrak{n}}{\mathfrak{d}}}\right) b$. By Corollary 5.2 , we obtain

$$
\phi_{\mathfrak{l}^{\prime}}\left(\tilde{x}_{\mathfrak{n}, \mathfrak{l}}\right)=-\sum_{\mathfrak{d} \mid \mathfrak{n}} a_{\mathfrak{d}} \otimes \tilde{\delta}_{\mathfrak{\mathfrak { d }}} \mathfrak{l}^{\prime}-\sum_{\mathfrak{d} \mid \mathfrak{n}} a_{\mathfrak{d}} \otimes \tilde{\delta}_{\mathfrak{\mathfrak { n }}} \otimes \phi_{\mathfrak{l}^{\prime}}(b),
$$

which completes the proof.

6.2. Recall that we took a generator $\sigma_{\mathfrak{r}}$ of $G_{\mathfrak{r}}$ for each $\mathfrak{r} \in \mathcal{S}$. We define $x_{\mathfrak{n}, \mathfrak{r}} \in\left(K^{\times} \otimes \mathbf{Z} / p^{N}\right)^{\chi}$ by

$$
\tilde{x}_{\mathfrak{n}, \mathfrak{l}}=x_{\mathfrak{n}, \mathfrak{l}} \otimes \otimes_{\mathfrak{r} \mid \mathfrak{n}} \sigma_{\mathfrak{r}}
$$

For $\mathfrak{l} \in \mathcal{S}(K(\mathfrak{n}))$, $\tilde{\kappa}_{\mathfrak{n}, \mathfrak{l}}$ was defined by $\tilde{\kappa}_{\mathfrak{n}, \mathfrak{l}}=\kappa_{\mathfrak{n}, \mathfrak{l}} \otimes \otimes_{\mathfrak{r} \mid \mathfrak{n}} \sigma_{\mathfrak{r}}$ in $\S 3$. For any $(\mathfrak{n}, \mathfrak{l})$ with $\mathfrak{n} \mathfrak{l} \in$ $\mathcal{N}_{[\epsilon(\mathfrak{n})]}(K)$, we define $\kappa_{\mathfrak{n}, \mathfrak{l}} \in\left(K^{\times} \otimes \mathbf{Z} / p^{N}\right)^{\chi}$ by

$$
\tilde{\kappa}_{\mathfrak{n}, \mathfrak{l}}=\kappa_{\mathfrak{n}, \mathfrak{l}} \otimes \otimes_{\mathfrak{r} \mid \mathfrak{n}} \sigma_{\mathfrak{r}}
$$

which is consistent with the definition in the case $\mathfrak{l} \in \mathcal{S}(K(\mathfrak{n}))$. We also use an element $\bar{a}_{\mathfrak{d}} \in$ $O_{\chi} / p^{N}[\Gamma(K / k)]$ which is defined by

$$
a_{\mathfrak{d}}=\bar{a}_{\mathfrak{d}} \otimes \otimes_{\mathfrak{r} \mid \mathfrak{d}} \sigma_{\mathfrak{r}}
$$

Recall that

$$
\bar{\phi}_{\mathfrak{r}}:\left(K^{\times} / p^{N}\right)^{\chi} \longrightarrow O_{\chi} / p^{N}[\Gamma(K / k)]
$$


is the homomorphism such that $\phi_{\mathfrak{r}}(x)=\bar{\phi}_{\mathfrak{r}}(x) \otimes \sigma_{\mathfrak{r}}$ for all $x$.

By Proposition 6.1 and Lemma 6.2 we have

Proposition 6.3. (0) If $\rho$ is a prime of $K$ which does not divide $\mathfrak{n} \mathfrak{l}$, it is not in the support of $\operatorname{div}\left(x_{\mathfrak{n}, \mathfrak{l}}\right)$.

(1) For each prime $\mathfrak{r}$ dividing $\mathfrak{n}$, we have $\operatorname{div}_{\mathfrak{r}}\left(x_{\mathfrak{n}, \mathfrak{l}}\right)=\bar{\phi}_{\mathfrak{r}}\left(x_{\frac{\mathfrak{n}}{\mathfrak{r}}, \mathfrak{l}}\right)$.

(2) For each prime $\mathfrak{r}$ dividing $\mathfrak{n}$, we have $\bar{\phi}_{\mathfrak{r}}\left(x_{\mathfrak{n}, \mathfrak{l}}\right)=\bar{a}_{\mathfrak{r}} \bar{\phi}_{\mathfrak{r}}\left(x_{\frac{\mathfrak{r}}{\mathfrak{r}}, \mathfrak{l}}\right)$.

(3) We take $\mathfrak{l}^{\prime}$ and $b$ as before Lemma 6.2. Then we have

$$
\bar{\phi}_{\mathfrak{l}^{\prime}}\left(x_{\mathfrak{n}, \mathfrak{l}}\right)=-\sum_{\mathfrak{d} \mid \mathfrak{n}} \bar{a}_{\mathfrak{d}}\left(\delta_{\frac{\mathfrak{n}}{\mathfrak{d}} \mathfrak{l}^{\prime}}+\delta_{\frac{\mathfrak{n}}{\mathfrak{d}}} \bar{\phi}_{\mathfrak{l}^{\prime}}(b)\right) .
$$

\section{Higher Stickelberger ideals}

Let $K, \chi, \ldots$ be as in the previous sections, namely as in $\S 3.1$ (recall that we are fixing an odd character $\chi$ of $\Delta(K / k))$, but in this section and the next section we do not assume $(*)$ in $§ 3.1$. In this section, we define the $i$-th Stickelberger ideal $\Theta_{i, K}^{\chi} \subset O_{\chi}[\Gamma(K / k)]$ of $K$ for all $i \in \mathbf{Z}_{\geq 0}$. More precisely, we define two ideals $\Theta_{i, K}^{(\delta), \chi}, \Theta_{i, K}^{\chi}$ such that $\Theta_{i, K}^{(\delta), \chi} \subset \Theta_{i, K}^{\chi}$. In the case that $K$ is a subfield of the cyclotomic $\mathbf{Z}_{p}$-extension of $K_{0}$ (namely, $K=K_{0, m}$ for some $m$ in the notation of $\S 1$ ), we will prove that they coincide in $\S 9$. In Remark 7.2, we will see that they do not coincide in general.

7.1. For $\mathfrak{n} \in \mathcal{N}(K)$, we consider $\delta_{\mathfrak{n}} \in O_{\chi} / p^{N}[\Gamma(K / k)]$, which is defined in $\S 3.3$ (note that this is defined without the assumption $(*)$ in $\S 3.1)$. We note that by definition all divisors $\mathfrak{l}$ of $\mathfrak{n} \in \mathcal{N}(K)$ satisfy $n_{\mathfrak{r}} \geq N$. When we would like to clarify this, we write $\mathcal{N}_{N}(K)$ for $\mathcal{N}(K)$. For $i \geq 0$, we define $\Theta_{i, K}^{(\delta, N), \chi}$ to be the ideal generated by

$$
\left\{\delta_{\mathfrak{n}} \mid \mathfrak{n} \in \mathcal{N}_{N}(K), \quad \epsilon(\mathfrak{n}) \leq i\right\}
$$

We define the small $i$-th Stickelberger ideal $\Theta_{i, K}^{(\delta), \chi}$ by

$$
\Theta_{i, K}^{(\delta), \chi}:=\lim _{\leftarrow N} \Theta_{i, K}^{(\delta, N), \chi} \subset \lim _{\leftarrow} O_{\chi} / p^{N}[\Gamma(K / k)]=O_{\chi}[\Gamma(K / k)] .
$$

In particular, $\Theta_{0, K}^{(\delta), \chi}$ is the principal ideal generated by $\theta_{K}^{\chi}$.

7.2. We define $\Theta_{i, K}^{\chi}$ by the same method as [10] $\S 3$. Let $s$ and $r$ be positive integers, and put $s^{\prime}=\min \left\{x \in \mathbf{Z}: s<p^{x}\right\}$. We consider a ring

$$
R=O_{\chi}[\Gamma(K / k)]\left[\left[S_{1}, \ldots, S_{r}\right]\right] /\left(\left(1+S_{1}\right)^{p^{n_{1}}}-1, \ldots,\left(1+S_{r}\right)^{p^{n_{r}}}-1\right)
$$

with $n_{1}, \ldots, n_{r} \geq N+s^{\prime}-1$. Let $f=\Sigma_{i_{1}, \ldots i_{r} \geq 0} a_{i_{1} \ldots i_{r}} S_{1}^{i_{1}} \ldots S_{r}^{i_{r}} \bmod \mathcal{I}$ be an element of $R$ where $a_{i_{1} \ldots i_{r}} \in O_{\chi}[\Gamma(K / k)]$ and $\mathcal{I}=\left(\left(1+S_{1}\right)^{p^{n_{1}}}-1, \ldots,\left(1+S_{r}\right)^{p^{n_{r}}}-1\right)$. Since $\operatorname{ord}_{p}\left(\left(\begin{array}{c}p^{n_{l}} \\ j\end{array}\right)\right)=$ $\operatorname{ord}_{p}\left(p^{n_{l}} ! /\left(j !\left(p^{n_{l}}-j\right) !\right)\right) \geq n_{l}-s^{\prime}+1$ for all $j$ with $0<j<p^{s^{\prime}} \quad(1 \leq l \leq r)$, considering the coefficients of the expansion $\left(1+S_{i}\right)^{p^{n_{l}}}-1$, we know $a_{i_{1}, \ldots, i_{r}} \bmod p^{q}$ with $q=$ $\min \left\{n_{1}, \ldots, n_{r}\right\}-s^{\prime}+1$ and $i_{1}, \ldots, i_{r} \leq s$ is well-defined, namely $a_{i_{1}, \ldots, i_{r}} \bmod p^{q}$ is determined by $f$. Hence $a_{i_{1}, \ldots, i_{r}} \bmod p^{N}$ is also well-defined. For $i \in \mathbf{Z}_{\geq 0}$ and $s \in \mathbf{Z}_{>0}$, we define $I_{i, s}(f)$ to be the ideal of $O_{\chi} / p^{N}[\Gamma(K / k)]$ generated by

$$
\left\{a_{i_{1}, \ldots, i_{r}} \bmod p^{N} \mid 0 \leq i_{1}, \ldots, i_{r} \leq s \text { and } i_{1}+\ldots+i_{r} \leq i\right\}
$$


Recall that $K_{0}$ is a subfield of $K$ such that $\Gamma(L / k)=\operatorname{Gal}\left(L / K_{0}\right)$ and $\Gamma(K / k)=\operatorname{Gal}\left(K / K_{0}\right)$. We define $\mathcal{F}^{\prime}$ by

$$
\begin{gathered}
\mathcal{F}^{\prime}=\left\{L_{0} \mid K_{0} \subset L_{0}, L_{0} / k \text { is finite and abelian, } L_{0} / K_{0} \text { is a } p \text {-extension, } L_{0} \cap K=K_{0},\right. \\
\text { and every prime above } \left.p \text { is unramified in } L_{0} / K_{0}\right\} .
\end{gathered}
$$

For $L_{0} \in \mathcal{F}^{\prime}$, we put $L=L_{0} K$, then $L \in \mathcal{F}$ where $\mathcal{F}$ is the set we defined in $\S 3.2$. We have a canonical isomorphism

$$
\Gamma(L / k)=\operatorname{Gal}\left(L / K_{0}\right)=\operatorname{Gal}(L / K) \times \operatorname{Gal}\left(L / L_{0}\right) \simeq \operatorname{Gal}(L / K) \times \Gamma(K / k) .
$$

We fix this isomorphism, and identify $O_{\chi}[\Gamma(L / k)]$ with $O_{\chi}[\Gamma(K / k)][\operatorname{Gal}(L / K)]$. For $s>0$, we put

$$
\begin{aligned}
\mathcal{F}_{s}^{\prime}=\left\{L_{0} \in \mathcal{F}^{\prime} \mid\right. & \operatorname{Gal}\left(L_{0} / K_{0}\right) \text { is of the form } \operatorname{Gal}\left(L_{0} / K_{0}\right)=\mathbf{Z} / p^{n_{1}} \mathbf{Z} \oplus \ldots \oplus \mathbf{Z} / p^{n_{r}} \mathbf{Z} \\
& \text { with } \left.n_{1}, \ldots, n_{r} \geq N+s^{\prime}-1 \text { for some } r>0\right\} \cup\left\{K_{0}\right\} .
\end{aligned}
$$

For $\mathfrak{n} \in \mathcal{N}(K)\left(=\mathcal{N}_{N}(K)\right)$, we have $K_{0}(\mathfrak{n}) \cap K=K_{0}$, so $K_{0}(\mathfrak{n})$ is in $\mathcal{F}^{\prime}$, and is in $\mathcal{F}_{1}^{\prime}$. Suppose that $L_{0}$ is in $\mathcal{F}_{s}^{\prime}, L=L_{0} K$ and $\operatorname{Gal}(L / K)=\operatorname{Gal}\left(L_{0} / K_{0}\right)=\mathbf{Z} / p^{n_{1}} \mathbf{Z} \oplus \ldots \oplus \mathbf{Z} / p^{n_{r}} \mathbf{Z}$. Fixing generators $\sigma_{1}, \ldots, \sigma_{r}$ of $\operatorname{Gal}(L / K)$, we have an isomorphism

$$
\begin{aligned}
O_{\chi}[\Gamma(L / k)] & =O_{\chi}[\Gamma(K / k)][\operatorname{Gal}(L / K)] \\
& \simeq O_{\chi}[\Gamma(K / k)]\left[\left[S_{1}, \ldots, S_{r}\right]\right] /\left(\left(1+S_{1}\right)^{p^{n_{1}}}-1, \ldots,\left(1+S_{r}\right)^{p^{n_{r}}}-1\right)
\end{aligned}
$$

where $\sigma_{l}$ corresponds to $1+S_{l}(1 \leq l \leq r)$. We regard $\theta_{L}^{\chi} \in O_{\chi}[\Gamma(L / k)]($ see $\S 2.4)$ as an element of the lower right ring, and define $I_{i, s}\left(\theta_{L}^{\chi}\right) \subset O_{\chi} / p^{N}[\Gamma(K / k)]$. It is easy to check that $I_{i, s}\left(\theta_{L}^{\chi}\right)$ does not depend on the choice of generators $\sigma_{1}, \ldots, \sigma_{r}$ of $\operatorname{Gal}(L / K)$ (see Lemma 3.1 in $[\mathbf{1 0}]$; note that the ideal generated by the coefficients of degree $i$ of $\theta_{L}^{\chi}$ depends on the choice of $\sigma_{1}, \ldots \sigma_{r}$, but the ideal $I_{i, s}\left(\theta_{L}^{\chi}\right)$ does not). We also note that this ideal $I_{i, s}\left(\theta_{L}^{\chi}\right)$ depends on the choice of $L_{0}$. We define $\Theta_{i, s, K}^{(N), \chi}$ to be the ideal of $O_{\chi} / p^{N}[\Gamma(K / k)]$ generated by

$$
\left\{I_{i, s}\left(\theta_{L}^{\chi}\right) \text { where } L=L_{0} K \mid L_{0} \in \mathcal{F}_{s}^{\prime}\right\}
$$

and $\Theta_{i, K}^{(N), \chi}$ to be the ideal generated by $\bigcup_{s>0} \Theta_{i, s, K}^{(N), \chi}$. In Theorem 8.11 we prove a relation between $\Theta_{i, K}^{(N), \chi}$ and the Fitting ideals. Finally, we define

$$
\Theta_{i, s, K}^{\chi}:=\lim _{\leftarrow} \Theta_{i, s, K}^{(N), \chi}, \quad \text { and } \Theta_{i, K}^{\chi}:=\lim _{\leftarrow N} \Theta_{i, K}^{(N), \chi} \subset O_{\chi}[\Gamma(K / k)] .
$$

Suppose $\mathfrak{n}=\mathfrak{r}_{1} \cdot \ldots \cdot \mathfrak{r}_{i} \in \mathcal{N}_{N}(K)$. We consider the isomorphism

$$
O_{\chi}[\Gamma(K(\mathfrak{n}) / k)]=O_{\chi}[\Gamma(K / k)]\left[\left[S_{1}, \ldots, S_{i}\right]\right] /\left(\left(1+S_{1}\right)^{p^{n_{\mathfrak{r}_{1}}}}-1, \ldots,\left(1+S_{i}\right)^{p^{n_{\mathfrak{r}_{i}}}}-1\right)
$$

defined by the correspondence $\sigma_{\mathfrak{r}_{l}} \leftrightarrow 1+S_{l}$. Then by Lemma 4.4 in [10] we have (cf. $\S 3.3$ )

$$
\theta_{K(\mathfrak{n})}^{\chi} \equiv(-1)^{i} \delta_{\mathfrak{n}} S_{1} \cdot \ldots \cdot S_{i} \quad\left(\bmod p^{N}, S_{1}^{2}, \ldots, S_{m}^{2}\right)
$$

Hence $\delta_{\mathfrak{n}}$ is in $\Theta_{i, 1, K}^{(N), \chi}$, and we obtain $\Theta_{i, K}^{(\delta, N), \chi} \subset \Theta_{i, 1, K}^{(N), \chi}$. Therefore, we have

$$
\Theta_{i, K}^{(\delta), \chi} \subset \Theta_{i, 1, K}^{\chi} \subset \Theta_{i, K}^{\chi}
$$

REMARK 7.1. Suppose that $L_{0}$ is in $\mathcal{F}^{\prime}$ and $L=L_{0} K$. We write $I_{L}$ for the kernel of the restriction map $O_{\chi}[\Gamma(L / k)] \longrightarrow O_{\chi}[\Gamma(K / k)]$. Suppose that $i_{L / K}: O_{\chi}[\Gamma(K / k)] \longrightarrow O_{\chi}[\Gamma(L / k)]$ is the natural map induced by the homomorphism $\Gamma(K / k) \longrightarrow \Gamma(K / k) \times \operatorname{Gal}(L / K)=\Gamma(L / k)$. We define the ideal $\tilde{\Theta}_{i, K}^{\chi}$ to be the minimal ideal in

$\left\{J\right.$ : ideal of $O_{\chi}[\Gamma(K / k)] \mid$ for any $L_{0} \in \mathcal{F}^{\prime}, \theta_{L}^{\chi} \in i_{L / K}(J) O_{\chi}[\Gamma(L / k)]+I_{L}^{i+1}$ where $\left.L=L_{0} K\right\}$. 
Then we can prove that $\Theta_{i, K_{0, m}}^{\chi}=\tilde{\Theta}_{i, K_{0, m}}^{\chi}$ for $K_{0, m}$ satisfying the assumptions of Theorem 1.4 (in fact, we can show that both are equal to the $i$-th Fitting ideal of $A_{K_{0, m}}^{\chi}$ ). So we could adopt the above definition of $\tilde{\Theta}_{i, K}^{\chi}$ as the definition of $\Theta_{i, K}^{\chi}$. Our definition of $\Theta_{i, K}^{\chi}$ is more useful for numerical computation.

REMARK 7.2. In general, we have $\Theta_{i, K}^{(\delta), \chi} \neq \Theta_{i, K}^{\chi}$. We will give examples for which $\Theta_{i, K}^{(\delta), \chi} \subsetneq$ $\Theta_{i, 1, K}^{\chi} \subset \Theta_{i, K}^{\chi}$. For simplicity, we assume $A_{k}=0, A_{K_{0}}^{\chi}=0, K_{0} \cap k\left(\mu_{p}\right)=k$, and $\mathfrak{l}_{0} \in \mathcal{S}\left(K_{0}\right)$ is a principal ideal. We consider $K=K_{0}\left(\mathfrak{l}_{0}\right)$, so $K / K_{0}$ is a $p$-extension such that $\operatorname{Gal}\left(K / K_{0}\right)=G_{\mathfrak{l}_{0}}$. Then by genus theory $A_{K}^{\chi}$ is generated by one element over $O_{\chi}[\Gamma(K / k)]=O_{\chi}\left[G_{\mathfrak{l}_{0}}\right]$ and $A_{K}^{\chi} \neq 0$ (cf. Proposition 5.2 in $[\mathbf{9}]$ ).

Concerning the Stickelberger ideals, we can first show that for any $\mathfrak{l} \in \mathcal{S}(K), \delta_{\mathfrak{l}}$ is not a unit in $O_{\chi} / p^{N}[\Gamma(K / k)]=O_{\chi} / p^{N}\left[G_{\mathfrak{l}_{0}}\right]$. In fact, we put $L=K(\mathfrak{l}), L_{0}=K_{0}(\mathfrak{l})$, and $S=\sigma_{\mathfrak{l}}-1 \in$ $O_{\chi} / p^{N}[\Gamma(L / k)]=O_{\chi} / p^{N}\left[G_{\mathfrak{l}_{0}} \times G_{\mathfrak{l}}\right]$. We know $\theta_{L}^{\chi} \equiv-\delta_{[} S \quad\left(\bmod p^{N}, S^{2}\right)$ by Lemma 4.4 in $[\mathbf{1 0}]$ ((3.3) in $\$ 2.3)$. On the other hand, by Lemma 2.4 we have $c_{L / L_{0}}\left(\theta_{L}^{\chi}\right)=\left(1-\varphi_{\mathrm{I}_{0}}^{-1}\right)^{\chi} \theta_{L_{0}}^{\chi}$ where $c_{L / L_{0}}: O_{\chi}[\Gamma(L / k)]=O_{\chi}\left[G_{\mathfrak{l}_{0}} \times G_{\mathfrak{l}}\right] \longrightarrow O_{\chi}\left[G_{\mathfrak{l}}\right]=O_{\chi}\left[\Gamma\left(L_{0} / k\right)\right]$ is the restriction map. Since $\mathfrak{l}_{0}$ splits completely in $K_{0}, \varphi_{\mathfrak{l}_{0}}=\left(\mathfrak{l}_{0}, L_{0} / k\right)$ is in $\operatorname{Gal}\left(L_{0} / K_{0}\right)=G_{\mathfrak{l}}$. We write $\varphi_{\mathfrak{l}_{0}}^{-1}=\sigma_{\mathfrak{l}}^{i}$ for some $i \in \mathbf{Z}$ in $\operatorname{Gal}\left(L_{0} / K_{0}\right)=G_{\mathfrak{l}}$. Combining two equations, we obtain

$$
-c_{L / L_{0}}\left(\delta_{\mathfrak{l}}\right) S \equiv\left(1-(1+S)^{i}\right) \theta_{L_{0}}^{\chi} \equiv-i \theta_{L_{0}}^{\chi} S \quad\left(\bmod p^{N}, S^{2}\right) .
$$

Applying Proposition 5.2 in [9] (or genus theory) also to $L_{0}$, we have $A_{L_{0}}^{\chi} \neq 0$. By Theorem 8.10 in the next section, $\theta_{L_{0}}^{\chi}$ is in $\operatorname{Fitt}_{0, O_{\chi}\left[G_{I}\right]}\left(A_{L_{0}}^{\chi}\right)$. Hence, $\theta_{L_{0}}^{\chi}$ is not a unit. It follows from the above congruence that $c_{L / L_{0}}\left(\delta_{\mathfrak{l}}\right)$ is not a unit, which shows that $\delta_{\mathfrak{l}}$ is not a unit.

Therefore, we have $\Theta_{1, K}^{(\delta), \chi} \subsetneq O_{\chi}\left[G_{\mathfrak{l}_{0}}\right]$ (namely $\Theta_{1, K}^{(\delta)}$ is too small).

Next, we consider $\Theta_{1, K}^{\chi}$. Suppose that $\mathfrak{l}_{0}=(x)$ for some $x \in k^{\times}$. By the Chebotarev density theorem, we can take $\mathfrak{r} \in \mathcal{S}$ such that $\mathfrak{r}$ is inert in $k(\sqrt[p]{x})$ and no prime above $\mathfrak{r}$ splits in $K_{0} / K_{0}^{+}$. We put $M=K(\mathfrak{r}), M_{0}=K_{0}(\mathfrak{r}), S=\sigma_{\mathfrak{r}}-1$, and write $\theta_{M}^{\chi} \equiv a_{0}+a_{1} S \quad\left(\bmod p^{N}, S^{2}\right)$ with $a_{0}, a_{1} \in O_{\chi} / p^{N}\left[G_{\mathfrak{l}_{0}}\right]$. Then we can check that $a_{1}$ is a unit. In fact, since $\mathfrak{r}$ is inert in $k(\sqrt[p]{x}), x \bmod \mathfrak{r}$ is not a $p$-th power in the residue field of $\mathfrak{r}$. By the Artin reciprocity law, we know that $\left(\mathfrak{l}_{0}, M_{0} / k\right)$ is not a $p$-th power in $\operatorname{Gal}\left(M_{0} / k\right)$. In the same way as above, we have $c_{M / M_{0}}\left(\theta_{M}^{\chi}\right)=\left(1-\varphi_{\mathrm{I}_{0}}^{-1}\right)^{\chi} \theta_{M_{0}}^{\chi}$ where $\varphi_{\mathrm{I}_{0}}=\left(\mathfrak{l}_{0}, M_{0} / k\right)$. We write $\varphi_{\mathrm{I}_{0}}^{-1}=\sigma_{\mathfrak{r}}^{j}$ for some $j$ which is prime to $p$. By the same method as above we have

$$
c_{M / M_{0}}\left(a_{0}+a_{1} S\right) \equiv-j \theta_{M_{0}}^{\chi} S \quad\left(\bmod p^{N}, S^{2}\right) .
$$

Since no prime above $\mathfrak{r}$ splits in $K_{0} / K_{0}^{+}$, we have $A_{M_{0}}^{\chi}=0$ by Proposition 5.2 in [9]. Again by Theorem $8.10(1), \theta_{M_{0}}^{\chi}$ has to be a unit in $O_{\chi}\left[G_{\mathfrak{r}}\right]$ (because $\nu_{1, \mathfrak{r}}\left(\theta_{K_{0}}^{\chi}\right)$ is not a unit). Therefore, $c_{M / M_{0}}\left(a_{0}\right) \equiv 0$ and $c_{M / M_{0}}\left(a_{1}\right)$ is a unit because both $j$ and $\theta_{M_{0}}^{\chi}$ are units. Hence, $a_{1}$ is a unit.

Since $M_{0} \in \mathcal{F}^{\prime}$, we obtain

$$
\Theta_{1, K}^{(\delta), \chi} \subsetneq \Theta_{1,1, K}^{\chi}=\Theta_{1, K}^{\chi}=O_{\chi}[\Gamma(K / k)] .
$$

For example, if $k=\mathbf{Q}, K_{0}=\mathbf{Q}(\sqrt{-6}), p=3, N=1, \mathfrak{l}_{0}=7, K=K_{0}\left(\mathfrak{l}_{0}\right)=K_{0}(\cos (2 \pi / 7))$, and $\chi$ is the nontrivial character of $\operatorname{Gal}\left(K_{0} / \mathbf{Q}\right)$, then all the assumptions we made are satisfied. We can take $M_{0}=K_{0}(\mathfrak{r})$ with $\mathfrak{r}=13 \in \mathcal{S}$, for example. In this case, $\theta_{M}^{\chi}$ can be computed as

$$
\theta_{M}^{\chi}=-\left(4 T+4 T^{2}\right)-\left(14+22 T+14 T^{2}\right) S-\left(8+12+8 T^{2}\right) S^{2}
$$

$\bmod \left((1+T)^{3}-1,(1+S)^{3}-1\right)$ where we took $1+S=\sigma_{\mathfrak{r}} \in G_{\mathfrak{r}}=(\mathbf{Z} / 13 \mathbf{Z})^{\times} \otimes \mathbf{Z} / 3 \mathbf{Z}$ which corresponds to $2 \otimes 1$, and $1+T=\sigma_{\mathfrak{l}_{0}} \in G_{\mathfrak{l}_{0}}=(\mathbf{Z} / 7 \mathbf{Z})^{\times} \otimes \mathbf{Z} / 3 \mathbf{Z}$ which corresponds to $3 \otimes 1$. In this example, $a_{1}=-\left(14+22 T+14 T^{2}\right)$ is certainly a unit in $O_{\chi} / p^{N}[\Gamma(K / k)]$. 


\section{Fitting ideals}

In this section, we describe known facts on Fitting ideals.

8.1. Suppose that $R$ is a commutative ring, and $M$ is a finitely presented $R$-module. By definition, we have an exact sequence

$$
R^{m} \stackrel{f}{\longrightarrow} R^{n} \longrightarrow M \longrightarrow 0
$$

of $R$-modules where $m$ and $n$ are positive integers. (If $m<n$, using a projection $R^{n} \longrightarrow R^{m}$, we can replace the above sequence by the exact sequence $R^{n} \longrightarrow R^{n} \longrightarrow M \longrightarrow 0$, so we may assume $m \geq n$.) For an integer $i \geq 0$ the $i$-th Fitting ideal of $M$ is defined to be the ideal of $R$ generated by all $(n-i) \times(n-i)$ minors of the matrix $A$ corresponding to $f$. If $i \geq n$, it is defined to be $R$. This definition depends only on $M$ and does not depend on the choice of $f$

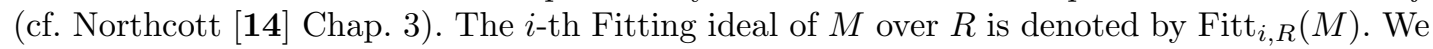
have a sequence of ideals

$$
\operatorname{Fitt}_{0, R}(M) \subset \operatorname{Fitt}_{1, R}(M) \subset \ldots \subset \operatorname{Fitt}_{n, R}(M)=\operatorname{Fitt}_{n+1, R}(M)=\ldots=R .
$$

The 0 -th Fitting ideal is called the initial Fitting ideal, and Fitt $_{i, R}(M)$ 's with $i \geq 1$ are called higher Fitting ideals.

These ideals give information on the structure of $M$ as an $R$-module. For example, by definition, if $\operatorname{Fitt}_{r, R}(M)=R, M$ is generated by at most $r$ elements.

8.2. In this subsection, we suppose that $O$ is a complete discrete valuation ring, and $\Lambda=O[[T]]$. Note that this is a noetherian unique factorial domain (Bourbaki [1] Chap. $7 \S 4$ Proposition 8). For finitely generated torsion $\Lambda$-modules $M_{1}$ and $M_{2}, M_{1}$ is said to be pseudo-isomorphic to $M_{2}$ if there is a $\Lambda$-homomorphism $M_{1} \longrightarrow M_{2}$ whose kernel and cokernel are both of finite length as $O$-modules. This is an equivalence relation of finitely generated torsion $\Lambda$-modules ([22] 1 13.2). We write $M_{1} \sim M_{2}$ in this case. If $M \sim 0, M$ is said to be a pseudo-null module. For any finitely generated torsion $\Lambda$-module $M$, there is a pseudo-isomorphism $M \sim \Lambda /\left(a_{1}\right) \oplus$ $\ldots \oplus \Lambda /\left(a_{r}\right)$ (Bourbaki [1] Chap. $7 \S 4$ Théorème 5). In this situation, the characteristic ideal $\operatorname{char}(M)$ is defined by $\operatorname{char}(M)=\left(a_{1} \cdot \ldots \cdot a_{r}\right)$.

The following lemma is well-known.

LEmma 8.1. Suppose that $M$ is a finitely generated torsion $\Lambda$-module, and it contains no nontrivial pseudo-null submodule.

(1) For any surjective $\Lambda$-homomorphism

$$
\varphi: \Lambda^{n} \longrightarrow M
$$

the kernel of $\varphi$ is a free $\Lambda$-module of rank $n$.

(2) $\operatorname{Fitt}_{0, \Lambda}(M)$ is a principal ideal.

(3) $\operatorname{Fitt}_{0, \Lambda}(M)$ is equal to the characteristic ideal char $(M)$.

Proof. (1) This follows from the fact that the projective dimension of $M$ is at most 1 (see for example, Wingberg [24] Proposition 2.1).

(2) We have an exact sequence $0 \longrightarrow \Lambda^{n} \stackrel{f}{\longrightarrow} \Lambda^{n} \longrightarrow M \longrightarrow 0$ by (1). Hence Fitt $0, \Lambda(M)$ is a principal ideal generated by $\operatorname{det} A$ where $A$ corresponds to $f$.

(3) Since $\operatorname{Fitt}_{0, \Lambda}(M)$ is generated by $\operatorname{det} A$, this follows from a well-known $\operatorname{property} \operatorname{char}(M)=$ (det $A)$ (Bourbaki [1] Chap. $7 \S 4$ Corollaire to Proposition 14). 
LEMMA 8.2. Let $M$ be a finitely generated torsion $\Lambda$-module such that

$$
M \sim \Lambda /\left(a_{1}\right) \oplus \ldots \oplus \Lambda /\left(a_{r}\right) \text { with }\left(a_{1}\right) \supset\left(a_{2}\right) \supset \ldots \supset\left(a_{r}\right) .
$$

Then $\operatorname{Fitt}_{i, \Lambda}(M)=\left(a_{1} \cdot \ldots \cdot a_{r-i}\right) I_{i}$ for all $i$ with $0 \leq i<r$ where $I_{i}$ is an ideal with length $_{O} \Lambda / I_{i}<\infty$. In particular, if we know all Fitt $_{i, \Lambda}(M)$, we get to know all $a_{j}(1 \leq j \leq r)$, namely we get to know the pseudo-isomorphism class of $M$.

Proof. In general, if there is an exact sequence $M_{1} \longrightarrow M_{2} \longrightarrow M_{3} \longrightarrow 0$ of $R$-modules, we have $\operatorname{Fitt}_{i, R}\left(M_{1}\right) \operatorname{Fitt}_{0, R}\left(M_{3}\right) \subset \operatorname{Fitt}_{i, R}\left(M_{2}\right)$ by elementary consideration of the matrix corresponding to $M_{2}([\mathbf{1 4}]$ page 91$)$.

Applying this to an exact sequence $M \longrightarrow \Lambda /\left(a_{1}\right) \oplus \ldots \oplus \Lambda /\left(a_{r}\right) \longrightarrow F \longrightarrow 0$ where $F$ is a pseudo-null $\Lambda$-module, we have

$$
\operatorname{Fitt}_{i, \Lambda}(M) \operatorname{Fitt}_{0, \Lambda}(F) \subset \operatorname{Fitt}_{i, \Lambda}\left(\Lambda /\left(a_{1}\right) \oplus \ldots \oplus \Lambda /\left(a_{r}\right)\right)=\left(a_{1} \cdot \ldots \cdot a_{r-i}\right) .
$$

Put $f_{i}=a_{1} \cdot \ldots \cdot a_{r-i}$. We take an arbitrary $x \in \operatorname{Fitt}_{i, \Lambda}(M)$. Since $\Lambda / F \operatorname{tit}_{0, \Lambda}(F)$ has finite length as an $O$-module, we can take $y_{1}, y_{2} \in \operatorname{Fitt}_{0, \Lambda}(F)$ such that $y_{1}$ and $y_{2}$ are relatively prime. It follows from the above inclusion that $f_{i}$ divides $x y_{1}$ and $x y_{2}$, and hence divides $x$. Therefore, $\operatorname{Fitt}_{i, \Lambda}(M) \subset\left(f_{i}\right)$, and we can write $\operatorname{Fitt}_{i, \Lambda}(M)=f_{i} I_{i}$ for some ideal $I_{i}$. We also have an exact sequence $\Lambda /\left(a_{1}\right) \oplus \ldots \oplus \Lambda /\left(a_{r}\right) \longrightarrow M \longrightarrow F^{\prime} \longrightarrow 0$ with length $_{O} F^{\prime}<\infty$, by which we obtain

$$
\operatorname{Fitt}_{i, \Lambda}\left(\Lambda /\left(a_{1}\right) \oplus \ldots \oplus \Lambda /\left(a_{r}\right)\right) \operatorname{Fitt}_{0, \Lambda}\left(F^{\prime}\right)=f_{i} \operatorname{Fitt}_{0, \Lambda}\left(F^{\prime}\right) \subset \operatorname{Fitt}_{i, \Lambda}(M)=f_{i} I_{i} .
$$

Hence $\operatorname{Fitt}_{0, \Lambda}\left(F^{\prime}\right) \subset I_{i}$. This shows that $\Lambda / I_{i}$ is of finite length as an $O$-module.

Concerning the isomorphism class of $M$, we have

LEMmA 8.3. Let $M$ be a finitely generated torsion $\Lambda$-module such that $M$ is free of rank 2 as an $O$-module. Then $\operatorname{Fitt}_{0, \Lambda}(M)$ and $\operatorname{Fitt}_{1, \Lambda}(M)$ determine the isomorphism class of $M$.

Proof. This is [9] Lemma 9.1.

REMARK 8.4. If $M$ is free of rank $r$ with $r>2$ as an $O$-module, Fitt $_{i, \Lambda}(M)$ 's do not determine the isomorphism class of $M$, in general.

For example, consider the $\Lambda$-modules $M_{1}, M_{2}$ corresponding to the matrices

$$
A_{1}=\left(\begin{array}{cc}
T^{2} & \pi^{2} \\
\pi^{3} & T
\end{array}\right), \quad A_{2}=\left(\begin{array}{cc}
T & \pi^{2} \\
\pi^{3} & T^{2}
\end{array}\right),
$$

respectively where $\pi$ is a prime element of $O$. Then both $M_{1}$ and $M_{2}$ are free of rank 3 as $O$ modules. Clearly, we get $\operatorname{Fitt}_{0, \Lambda}\left(M_{1}\right)=\operatorname{Fitt}_{0, \Lambda}\left(M_{2}\right)=\left(T^{3}-\pi^{5}\right), \operatorname{Fitt}_{1, \Lambda}\left(M_{1}\right)=\operatorname{Fitt}_{1, \Lambda}\left(M_{2}\right)=$ $\left(T, \pi^{2}\right)$, and $\operatorname{Fitt}_{i, \Lambda}\left(M_{1}\right)=\operatorname{Fitt}_{i, \Lambda}\left(M_{2}\right)=\Lambda$ for all $i \geq 2$. But $M_{1}$ is not isomorphic to $M_{2}$. In fact, put $I=\left(\pi^{3}, \pi T, T^{2}\right) \subset \Lambda$. Then we have length $\Lambda / I=4$, length $M_{1} / I M_{1}=4+4-1=$ 7 , and length $M_{2} / I M_{2}=4-2+4=6$.

LEMMA 8.5. We assume that $\psi: \Lambda \longrightarrow O_{\psi}$ is a surjective ring homomorphism such that $O_{\psi}$ is a discrete valuation ring. For a finitely generated torsion $\Lambda$-module $M$, we define $M^{\psi}=$ $M \otimes_{\Lambda} O_{\psi}$ and $J_{i}^{\psi}=\psi\left(\operatorname{Fitt}_{i, \Lambda}(M)\right)$. Then we have an isomorphism

$$
M^{\psi} \simeq \bigoplus_{i \geq 1} J_{i}^{\psi} / J_{i-1}^{\psi}
$$


as $O_{\psi}$-modules. Namely, if we know all $\mathrm{Fitt}_{i, \Lambda}(M)$ for $i \geq 0$, we get to know the isomorphism class of $M^{\psi}$ for all $\psi$.

Proof. In fact, by the definition of the Fitting ideals, we have $J_{i}^{\psi}=\operatorname{Fitt}_{i, O_{\psi}}\left(M^{\psi}\right)$. If $M^{\psi}$ is isomorphic to $O_{\psi} /\left(a_{1}\right) \oplus \ldots \oplus O_{\psi} /\left(a_{r}\right)$ such that $\left(a_{1}\right) \supset\left(a_{2}\right) \supset \ldots \supset\left(a_{r}\right)$, we have Fitt $_{i, O_{\psi}}\left(M^{\psi}\right)=\left(a_{1} \cdot \ldots \cdot a_{r-i}\right)$. Therefore, we obtain the isomorphism stated in Lemma 8.5.

8.3. Let $K, K_{0}, \chi$ etc be as before, namely as in $\S 3.1$ (but we do not assume $(*)$ in $\S 3.1$ ). From now on, we also assume that $K$ is in the cyclotomic $\mathbf{Z}_{p}$-extension $\left(K_{0}\right)_{\infty}$ of $K_{0}$. We denote by $K_{0, m}$ the intermediate field of $\left(K_{0}\right)_{\infty} / K_{0}$ such that $\left[K_{0, m}: K_{0}\right]=p^{m}$. Our assumption means $K=K_{0, m}$ for some $m \geq 0$. By definition, $K_{\infty}=\left(K_{0}\right)_{\infty}$. We study $X_{K_{\infty}}^{\chi}=\lim A_{K_{n}}^{\chi}$. Recall

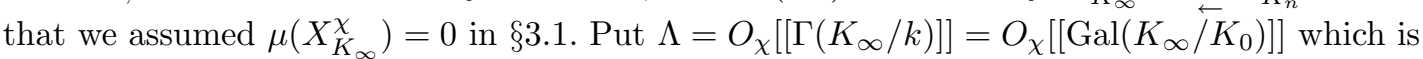
isomorphic to the formal power series ring $O_{\chi}[[T]]$, and we can apply the results in the previous subsection.

By Lemma 2.4, $\theta_{K_{n}}^{\chi}$ 's $\left(\in O_{\chi}\left[\operatorname{Gal}\left(K_{n} / K_{0}\right)\right]\right)$ for $n \gg 0$ become a projective system and we define $\theta_{K_{\infty}}^{\chi} \in O_{\chi}\left[\left[\operatorname{Gal}\left(K_{\infty} / K_{0}\right)\right]\right]=\Lambda$ as their projective limit which is the $p$-adic $L$-function of Deligne and Ribet. Since $X_{K_{\infty}}^{\chi}$ does not have a non-trivial finite $\Lambda$-submodule ([22] Proposition 13.28), by the main conjecture proved by Wiles [23] and Lemma 8.1 (3), we know

TheOrem 8.6. (Wiles $[\mathbf{2 3}]) \operatorname{Fitt}_{0, \Lambda}\left(X_{K_{\infty}}^{\chi}\right)=\left(\theta_{K_{\infty}}^{\chi}\right)$.

For any $\mathfrak{n} \in \mathcal{N}$, we put $K(\mathfrak{n})=K k(\mathfrak{n})$ and consider the cyclotomic $\mathbf{Z}_{p}$-extension $K(\mathfrak{n})_{\infty}$ of $K(\mathfrak{n})$. The element $\theta_{K(\mathfrak{n})_{\infty}}^{\chi} \in O_{\chi}\left[\left[\operatorname{Gal}\left(K(\mathfrak{n})_{\infty} / K_{0}\right)\right]\right]=\Lambda\left[G_{\mathfrak{n}}\right]$ is defined by the same method as above. For $\mathfrak{d} \in \mathcal{N}$ dividing $\mathfrak{n}$, we define the norm map

$$
\nu_{\mathfrak{d}, \mathfrak{n}}: \Lambda\left[G_{\mathfrak{d}}\right] \longrightarrow \Lambda\left[G_{\mathfrak{n}}\right]
$$

by $\Sigma_{\sigma \in G_{\mathfrak{d}}} a_{\sigma} \sigma \mapsto \Sigma_{\sigma \in G_{\mathfrak{d}}} a_{\sigma} \tau^{\prime}$ where $\tau^{\prime}=\Sigma_{\tau_{\mid K(\mathfrak{d})} \infty}=\sigma \quad \tau$ (the sum is taken over all $\tau \in G_{\mathfrak{n}}$ whose restriction to $K(\mathfrak{d})_{\infty}$ is $\left.\sigma\right)$. Since $K_{0}(\mathfrak{n})$ satisfies the condition $\left(\mathrm{A}_{p}\right)$ in $[\mathbf{9}]$, we have the following.

Theorem 8.7. ([9] Theorem 0.9)

$$
\operatorname{Fitt}_{0, \Lambda\left[G_{\mathfrak{n}}\right]}\left(X_{K(\mathfrak{n})_{\infty}}^{\chi}\right)=\left(\left\{\nu_{\mathfrak{d}, \mathfrak{n}}\left(\theta_{K(\mathfrak{d})_{\infty}}^{\chi}\right) \mid \mathfrak{d} \in \mathcal{N}, \mathfrak{d} \text { divides } \mathfrak{n}\right\}\right)
$$

where the right hand side is the ideal of $\Lambda\left[G_{\mathfrak{n}}\right]$ generated by all $\nu_{\mathfrak{d}, \mathfrak{n}}\left(\theta_{K(\mathfrak{d})_{\infty}}^{\chi}\right)$ 's.

We note that the Leopoldt conjecture is not needed in the proof of the above theorem because of $\chi \neq \omega$ (cf. Remark 0.11 (1) in [9]). We also note that $\operatorname{Fitt}_{0, \Lambda}\left(X_{K_{\infty}}^{\chi}\right)$ is a principal ideal, but Fitt $_{0, \Lambda\left[G_{\mathfrak{n}}\right]}\left(X_{K(\mathfrak{n})_{\infty}}^{\chi}\right)$ is not principal, in general.

In [4], Greither generalized the above theorem, and determined the initial Fitting ideal for more general cyclotomic $\mathbf{Z}_{p}$-extensions. By Greither [4] Theorem 7 (i), $\operatorname{Fitt}_{0, \Lambda}\left(X_{L_{\infty}}^{\chi}\right)$ is determined where $L=L_{0} K$ with $L_{0} \in \mathcal{F}^{\prime}$ (which was defined in $\S 7.2$ ).

In particular, we have the following.

Theorem 8.8. (Greither $[\mathbf{4}]$ Theorem 7 (i)) For any $L=L_{0} K$ with $L_{0} \in \mathcal{F}^{\prime}$, we have

$$
\theta_{L_{\infty}}^{\chi} \in \operatorname{Fitt}_{0, \Lambda[\operatorname{Gal}(L / K)]}\left(X_{L_{\infty}}^{\chi}\right)
$$


Recall that $L$ in the above theorem satisfies the condition that all primes of $K_{\infty}$ above $p$ are unramified in $L_{\infty}$. If we remove this assumption on the unramifiedness, there exist counterexamples of the above property ([5] Theorem 1.1).

8.4. We next study $A_{K}^{\chi}$ and $A_{L}^{\chi}$ for $L \in \mathcal{F}$.

LEMMA 8.9. The norm map induces an isomorphism

$$
\left(X_{L_{\infty}}^{\chi}\right)_{\operatorname{Gal}\left(L_{\infty} / L\right)} \stackrel{\simeq}{\longrightarrow} A_{L}^{\chi}
$$

for any $L \in \mathcal{F}$.

Proof. In fact, by our assumption that $\chi(\mathfrak{p}) \neq 1$ for all primes $\mathfrak{p}$ of $k$ above $p$, we have $\left(\bigoplus_{v \mid p} \mathbf{Z}_{p}\right)^{\chi}=0$ where $v$ ranges over primes of $K_{0}$ above $p$. Hence we also have $\left(\bigoplus_{w \mid p} \mathbf{Z}_{p}\right)^{\chi}=0$ where $w$ ranges over primes of $L$ above $p$, and $\left(\bigoplus_{w \mid p} I_{w}\left(L_{\infty} / L\right)\right)^{\chi}=0$ where $I_{w}\left(L_{\infty} / L\right)$ is the inertia group of $w$ in $\operatorname{Gal}\left(L_{\infty} / L\right)$. Therefore, by Proposition 5.2 in [9], we get the above isomorphism.

Suppose $L \in \mathcal{F}$. For a prime $\mathfrak{p}$ of $k$ above $p$, by our assumption $\chi(\mathfrak{p}) \neq 1,1-\chi(\mathfrak{p})^{-1}$ is a unit of $O_{\chi}$. Hence, if $\mathfrak{p}$ is unramified in $L,\left(1-\varphi_{\mathfrak{p}}^{-1}\right)^{\chi}$ is a unit of $O_{\chi}[\Gamma(L / k)]$ where $\varphi_{\mathfrak{p}}$ is the Frobenius of $\mathfrak{p}$ in $\operatorname{Gal}(L / k)$. Therefore, by Lemma 2.4, we have

$$
c_{L_{\infty} / L}\left(\theta_{L_{\infty}}^{\chi}\right)=u \theta_{L}^{\chi}
$$

for some $u \in O_{\chi}[\Gamma(L / k)]^{\times}$.

Put $R_{K}=O_{\chi}[\Gamma(K / k)]$. For $\mathfrak{n}, \mathfrak{d} \in \mathcal{N}$ with $\mathfrak{d} \mid \mathfrak{n}$, we define the norm map $\nu_{\mathfrak{d}, \mathfrak{n}}: R_{K}\left[G_{\mathfrak{d}}\right] \longrightarrow$ $R_{K}\left[G_{\mathfrak{n}}\right]$ by the same method as above, and consider $\nu_{\mathfrak{d}, \mathfrak{n}}\left(\theta_{K(\mathfrak{d})}^{\chi}\right) \in R_{K}\left[G_{\mathfrak{n}}\right]=O_{\chi}[\Gamma(K(\mathfrak{n}) / k)]$.

In general, for any ideal $I$ of $R$ and an $R$-module $M$, by the definition of the Fitting ideals,

$$
\operatorname{Fitt}_{i, R / I}(M / I M)=\operatorname{Fitt}_{i, R}(M) \bmod I \subset R / I
$$

holds. Therefore, using Theorems 8.7, 8.8 and Lemma 8.9, we obtain

Theorem 8.10. (1) For any $\mathfrak{n} \in \mathcal{N}$, we have

$$
\operatorname{Fitt}_{0, R_{K}\left[G_{\mathfrak{n}}\right]}\left(A_{K(\mathfrak{n})}^{\chi}\right)=\left(\left\{\nu_{\mathfrak{d}, \mathfrak{n}}\left(\theta_{K(\mathfrak{d})}^{\chi}\right) \mid \mathfrak{d} \in \mathcal{N}, \mathfrak{d} \text { divides } \mathfrak{n}\right\}\right)
$$

where the right hand side is the ideal of $R_{K}\left[G_{\mathfrak{n}}\right]=O_{\chi}[\Gamma(K(\mathfrak{n}) / k)]$ generated by all $\nu_{\mathfrak{d}, \mathfrak{n}}\left(\theta_{K(\mathfrak{d})}^{\chi}\right)$ 's. (2) For any $L=L_{0} K$ with $L_{0} \in \mathcal{F}^{\prime}$, we have $\theta_{L}^{\chi} \in \operatorname{Fitt}_{0, R_{K}[\operatorname{Gal}(L / K)]}\left(A_{L}^{\chi}\right)$.

8.5. Let $K$ be as above. We defined $\Theta_{i, K}^{\chi}$ in $\S 7.2$. In this subsection, we prove

Theorem 8.11. (cf. [9] Theorem 8.1) For any $i \geq 0$, we have

$$
\Theta_{i, K}^{(N), \chi} \subset \operatorname{Fitt}_{i, R_{K} / p^{N}}\left(A_{K}^{\chi} / p^{N}\right) \text { and } \Theta_{i, K}^{\chi} \subset \operatorname{Fitt}_{i, R_{K}}\left(A_{K}^{\chi}\right) .
$$

Proof. This is essentially Theorem 8.1 in [9]. Suppose that $L=L_{0} K$ with $L_{0} \in \mathcal{F}_{s}^{\prime}$ for some $s>0$, and $\operatorname{Gal}(L / K)=\mathbf{Z} / p^{n_{1}} \mathbf{Z} \oplus \ldots \oplus \mathbf{Z} / p^{n_{r}} \mathbf{Z}$ for some $r>0$. Put $G=\operatorname{Gal}(L / K)$. We take generators $\sigma_{1}, \ldots, \sigma_{r}$ of $G$, and identify $O_{\chi}[\Gamma(L / k)]=R_{K}[G]$ with

$$
R_{K}\left[\left[S_{1}, \ldots, S_{r}\right]\right] /\left(\left(1+S_{1}\right)^{p^{n_{1}}}-1, \ldots,\left(1+S_{r}\right)^{p^{n_{r}}}-1\right)
$$


by $\sigma_{l} \leftrightarrow 1+S_{l}(1 \leq l \leq r)$. By the definition of $\mathcal{F}_{s}^{\prime}$ and the consideration in $\S 7.2$, we have an isomorphism

$$
R_{K} / p^{N}[G] /\left(S_{1}^{s+1}, \ldots, S_{r}^{s+1}\right) \simeq R_{K} / p^{N}\left[\left[S_{1}, \ldots, S_{r}\right]\right] /\left(S_{1}^{s+1}, \ldots, S_{r}^{s+1}\right) .
$$

We regard $A_{K}^{\chi}$ as an $R_{K}[G]$-module, on which $G$ acts trivially. Let $R_{K}^{m} \stackrel{g}{\longrightarrow} R_{K}^{n} \longrightarrow A_{K}^{\chi} \longrightarrow 0$ be an exact sequence of $R_{K}$-modules, and $B$ be the matrix with $m$ columns and $n$ rows corresponding to $g$. We have an exact sequence $R_{K}[G]^{m+r n} \stackrel{g^{\prime}}{\longrightarrow} R_{K}[G]^{n} \longrightarrow A_{K}^{\chi} \longrightarrow 0$ of $R_{K}[G]$-modules where $g^{\prime}$ corresponds to the matrix

$$
\left(\begin{array}{ccccccccc}
S_{1} & \ldots & S_{r} & \ldots & \ldots & 0 & \ldots & 0 & \\
0 & \ldots & 0 & \ldots & \ldots & 0 & \ldots & 0 & \\
. & \ldots & . & \ldots & \ldots & . & \ldots & . & B \\
. & \ldots & . & \ldots & \ldots & . & \ldots & . & \\
0 & \ldots & 0 & \ldots & \ldots & S_{1} & \ldots & S_{r} &
\end{array}\right)
$$

Then we know from the above matrix that

$$
\operatorname{Fitt}_{0, R_{K}[G]}\left(A_{K}^{\chi}\right)=\sum_{i=0}^{n} \operatorname{Fitt}_{i, R_{K}}\left(A_{K}^{\chi}\right)\left(S_{1}, \ldots, S_{r}\right)^{i} .
$$

Since we have a surjective homomorphism $A_{L}^{\chi} \longrightarrow A_{K}^{\chi}$ of $R_{K}[G]$-modules ([9] Lemma $5.1(1)$ ), we have

$$
\operatorname{Fitt}_{0, R_{K}[G]}\left(A_{L}^{\chi}\right) \subset \sum_{i=0}^{n} \operatorname{Fitt}_{i, R_{K}}\left(A_{K}^{\chi}\right)\left(S_{1}, \ldots, S_{r}\right)^{i}
$$

This implies

$$
\operatorname{Fitt}_{0, R_{K}[G]}\left(A_{L}^{\chi}\right) \bmod \left(p^{N}, S_{1}^{s+1}, \ldots, S_{r}^{s+1}\right) \subset \sum_{i=0}^{n} \operatorname{Fitt}_{i, R_{K} / p^{N}}\left(A_{K}^{\chi} / p^{N}\right)\left(S_{1}, \ldots, S_{r}\right)^{i}
$$

in $R_{K} / p^{N}\left[\left[S_{1}, \ldots, S_{r}\right]\right] /\left(S_{1}^{s+1}, \ldots, S_{r}^{s+1}\right)$. By Theorem $8.10(2)$, we have $\theta_{L}^{\chi} \in \operatorname{Fitt}_{0, R_{K}[G]}\left(A_{L}^{\chi}\right)$, hence we obtain $I_{i, s}\left(\theta_{L}^{\chi}\right) \subset \operatorname{Fitt}_{i, R_{K} / p^{N}}\left(A_{K}^{\chi} / p^{N}\right)$. Thus, we have

$$
\Theta_{i, K}^{(N), \chi} \subset \operatorname{Fitt}_{i, R_{K} / p^{N}}\left(A_{K}^{\chi} / p^{N}\right) .
$$

Since

$$
\operatorname{Fitt}_{i, R_{K}}\left(A_{K}^{\chi}\right)=\lim _{\leftarrow N} \operatorname{Fitt}_{i, R_{K} / p^{N}}\left(A_{K}^{\chi} / p^{N}\right)
$$

from the definition of $\Theta_{i, K}^{\chi}$ we obtain the conclusion $\Theta_{i, K}^{\chi} \subset \operatorname{Fitt}_{i, R_{K}}\left(A_{K}^{\chi}\right)$.

We define the Stickelberger ideals over $K_{\infty}$ by

$$
\Theta_{i, K_{\infty}}^{(\delta), \chi}=\lim _{\leftarrow} \Theta_{i, K_{m}}^{(\delta), \chi} \text { and } \Theta_{i, K_{\infty}}^{\chi}=\lim _{\leftarrow} \Theta_{i, K_{m}}^{\chi} .
$$

From the inclusion $\Theta_{i, K_{m}}^{(\delta), \chi} \subset \Theta_{i, K_{m}}^{\chi}$, we know $\Theta_{i, K_{\infty}}^{(\delta), \chi} \subset \Theta_{i, K_{\infty}}^{\chi} \subset \Lambda$.

Corollary 8.12. For any $i \geq 0$, we have

$$
\Theta_{i, K_{\infty}}^{(\delta), \chi} \subset \Theta_{i, K_{\infty}}^{\chi} \subset \operatorname{Fitt}_{i, \Lambda}\left(X_{K_{\infty}}^{\chi}\right) .
$$

Proof. Suppose that $0 \longrightarrow \Lambda^{n} \stackrel{f}{\longrightarrow} \Lambda^{n} \longrightarrow X_{K_{\infty}}^{\chi} \longrightarrow 0$ is exact, and $\gamma_{m}$ is a generator of $\operatorname{Gal}\left(K_{\infty} / K_{m}\right)$. Then by Lemma $8.9 f \bmod \gamma_{m}-1$ yields an exact sequence $O_{\chi}\left[\Gamma\left(K_{m} / k\right)\right]^{n} \stackrel{\bmod \gamma_{m}-1}{\longrightarrow} O_{\chi}\left[\Gamma\left(K_{m} / k\right)\right]^{n} \longrightarrow A_{K_{m}}^{\chi} \longrightarrow 0$. Hence we have

$$
\operatorname{Fitt}_{i, \Lambda}\left(X_{K_{\infty}}^{\chi}\right)=\lim _{\leftarrow} \operatorname{Fitt}_{i, O_{\chi}\left[\Gamma\left(K_{n} / k\right)\right]}\left(A_{K_{n}}^{\chi}\right) \text {. }
$$


Therefore, Theorem 8.11 implies Corollary 8.12.

Concerning the commutativity of projective limits with Fitting ideals in a more general setting, see [5] Theorem 2.1.

\section{Proof of the Main Theorem}

In this section, we prove Theorem 1.1. In order to get the equality of two ideals, since we saw in the previous section that one inclusion holds (Corollary 8.12), we have to prove the other inclusion. Using $x_{\mathfrak{n}, \mathfrak{l}}$ in $\S 6$, we will construct elements in the multiplicative group which give relations approximating submatrices of a relation matrix of $X_{K_{\infty}}^{\chi}$. The properties of Kolyvagin systems ((iii) and (iv) in $\S 0$; more directly Proposition 6.3) play an important role (see Lemma 9.2 which is a key lemma).

9.1. For each $\mathfrak{l} \in \mathcal{S}$, using the prime $\mathfrak{l}_{\bar{k}}$ we fixed, we regard $\mu_{p^{n}} \subset \bar{k}^{\times}$as a subgroup of $\bar{k}_{\mathfrak{l}}^{\times}$for all $n>0$ where $\bar{k}_{\mathrm{l}}$ is an algebraic closure of $k_{\mathfrak{l}}$. We fix a generator $\zeta_{p^{n}} \in \mu_{p^{n}}$ for all $n>0$ such that $\left(\zeta_{p^{n+1}}\right)^{p}=\zeta_{p^{n}}$. For each $\mathfrak{l} \in \mathcal{S}$, we take $\sigma_{\mathfrak{l}} \in G_{\mathfrak{l}}$ to be the element such that $\operatorname{Kum}\left(\sigma_{\mathfrak{l}}\right)=\zeta_{p^{n} \mathfrak{l}}$ where Kum is the map defined in $\S 2.3$.

In the proof of the main theorem, we need the following lemma which is Rubin Theorem 3.1 in [15] combined with Lemma 4.5.

Lemma 9.1. Assume $\mathfrak{n}=\mathfrak{r}_{1} \cdot \ldots \cdot \mathfrak{r}_{m} \in \mathcal{N}(K)$ and $\mathfrak{l} \in \mathcal{S}(K)$ is prime to $\mathfrak{n}$. Suppose one is given $\sigma_{i} \in O_{\chi} / p^{N}[\Gamma(K / k)] \otimes G_{\mathfrak{r}_{i}}$ for each $i=1, \ldots, m$, a finite $\operatorname{Gal}(K / k)$-submodule $W$ of $\left(K^{\times} / p^{N}\right)^{\chi}$, and a $\operatorname{Gal}(K / k)$-equivariant homomorphism

$$
\lambda: W \longrightarrow O_{\chi} / p^{N}[\Gamma(K / k)] .
$$

Then there are infinitely many $\mathfrak{l}^{\prime} \in \mathcal{S}(K(\mathfrak{n}))$ which satisfy the following properties.

(i) The class $\left[\mathfrak{l}_{K}^{\prime}\right]^{\chi}$ in $A_{K}^{\chi}$ coincides with the class $\left[\mathfrak{l}_{K}\right]^{\chi}$.

(ii) For the element $z \in\left(K^{\times} \otimes \mathbf{Z}_{p}\right)^{\chi}$ such that $\operatorname{div}(z)=\left(\mathfrak{l}_{K}^{\prime}-\mathfrak{l}_{K}\right)^{\chi}, \phi_{\mathfrak{r}_{i}}(z)=\sigma_{i}$ holds for each $i=1, \ldots, m$.

(iii) $W$ is in the kernel of $\operatorname{div}_{\mathfrak{l}^{\prime}}:\left(K^{\times} / p^{N}\right)^{\chi} \longrightarrow O_{\chi} / p^{N}[\Gamma(K / k)]$, and we have $\lambda(x)=\bar{\phi}_{\mathfrak{l}^{\prime}}(x)$ for all $x \in W$.

Proof. We follow the argument of the proof of Rubin [15] Theorem 3.1, and our proof is a modification of [15] Theorem 3.1. So the reader who is not familiar with this kind of proof should consult the proof of [15] Theorem 3.1. Put $G=\operatorname{Gal}(K / k)$. We define $\iota: \mathbf{Z} / p^{N}[G] \longrightarrow$ $\mathbf{Z} / p^{N}$ by $a_{1} 1_{G}+\sum_{g \neq 1} a_{g} g \mapsto a_{1}$. Then $f \mapsto \iota \circ f$ defines an isomorphism

$$
\mathcal{I}: \operatorname{Hom}_{\mathbf{Z} / p^{N}[G]}\left(M, \mathbf{Z} / p^{N}[G]\right) \stackrel{\simeq}{\longrightarrow} \operatorname{Hom}\left(M, \mathbf{Z} / p^{N}\right)
$$

for any $\mathbf{Z} / p^{N}[G]$-module $M$. In fact, $f \mapsto\left(x \mapsto \Sigma_{\sigma \in G} f(\sigma x) \sigma^{-1}\right)$ gives the inverse of $\mathcal{I}$. Let $\zeta_{p^{N}}$ be the primitive $p^{N}$-th root of unity we fixed. We regard $W$ as a $\mathbf{Z} / p^{N}[G]$-module, $O_{\chi} / p^{N}[\Gamma(K / k)]$ as a direct summand of $\mathbf{Z} / p^{N}[G]$, and $\lambda$ as a map from $W$ to $\mathbf{Z} / p^{N}[G]$. We define $\lambda^{\prime}: W \longrightarrow \mu_{p^{N}}$ by $\lambda^{\prime}(x)=\zeta_{p^{N}}^{-(\llcorner 0 \lambda)(x)}$.

Consider the Kummer pairing

$$
\operatorname{Gal}\left(K\left(\mu_{p^{N}}, W^{1 / p^{N}}\right) / K\left(\mu_{p^{N}}\right)\right) \times W \longrightarrow \mu_{p^{N}}
$$

which is non-degenerate because of the injectivity of $\left(K^{\times} / p^{N}\right)^{\chi} \longrightarrow\left(K\left(\mu_{p^{N}}\right)^{\times} / p^{N}\right)^{\chi}$. (Here, we regard $\chi$ as a character of $\Delta\left(K\left(\mu_{p^{N}}\right) / k\right)$ using the natural restriction $\Delta\left(K\left(\mu_{p^{N}}\right) / k\right) \longrightarrow$ $\Delta(K / k)$.) Using this pairing, we regard $\lambda^{\prime}$ as an element of $\operatorname{Gal}\left(K\left(\mu_{p^{N}}, W^{1 / p^{N}}\right) / K\left(\mu_{p^{N}}\right)\right)$. 
Let $K\{\mathfrak{n}\}^{\chi}$ be as in the proof of Lemma 4.5 , and let $\mathcal{U}$ and $k\left(\mu_{p^{n+1}}, \mathcal{U}^{1 / p^{n}}\right)$ be as in the proof of Lemma 2.1. We consider the compositum

$$
L^{\prime}=K\{\mathfrak{n}\}^{\chi} K(\mathfrak{n})\left(\mu_{p^{n+1}}, \mathcal{U}^{1 / p^{n}}, W^{1 / p^{N}}\right) .
$$

The Galois group $\operatorname{Gal}\left(K_{0}\left(\mu_{p}\right) / k\right)$ acts on $\operatorname{Gal}\left(K\{\mathfrak{n}\}^{\chi} / K\right)$ via $\chi$, acts on $\operatorname{Gal}\left(K(\mathfrak{n})\left(\mu_{p^{n+1}}\right) / K\right)$ via the trivial character, acts on $\operatorname{Gal}\left(K\left(\mu_{p^{n}}, \mathcal{U}^{1 / p^{n}}\right) / K\left(\mu_{p^{n}}\right)\right)$ via $\omega$, and acts on $\operatorname{Gal}\left(K\left(\mu_{p^{N}}, W^{1 / p^{N}}\right) / K\left(\mu_{p^{N}}\right)\right)$ via $\chi^{-1} \omega$. Hence $K\{\mathfrak{n}\}^{\chi}\left(\mu_{p^{n}}\right), K(\mathfrak{n})\left(\mu_{p^{n}}\right), K\left(\mu_{p^{n+1}}\right), K\left(\mu_{p^{n}}, \mathcal{U}^{1 / p^{n}}\right)$, and $K\left(\mu_{p^{n}}, W^{1 / p^{N}}\right)$ are all linearly disjoint over $K\left(\mu_{p^{n}}\right)$. Hence, as in the proof of Lemma 4.5, we can apply the Chebotarev density theorem to $L^{\prime}$, and obtain infinitely many $\mathfrak{l}^{\prime} \in \mathcal{S}(K(\mathfrak{n}))$ having the properties (i), (ii) and $\left(\mathfrak{l}_{K\left(\mu_{\left.p^{n}\right)}\right.}^{\prime}, K\left(\mu_{p^{n}}, W^{1 / p^{N}}\right) / K\left(\mu_{p^{n}}\right)\right)=\lambda^{\prime}$.

Since $\mathfrak{l}^{\prime}$ is unramified in $K\left(\mu_{p^{n}}, W^{1 / p^{N}}\right), W$ is in the kernel of $\operatorname{div}_{\mathfrak{l}^{\prime}}:\left(K^{\times} / p^{N}\right)^{\chi} \longrightarrow$ $O_{\chi} / p^{N}[\Gamma(K / k)]$. We write $\varphi_{\mathfrak{l}^{\prime}}=\left(\mathfrak{l}_{K\left(\mu_{p^{n}}\right)}^{\prime}, K\left(\mu_{p^{n}}, W^{1 / p^{N}}\right) / K\left(\mu_{p^{n}}\right)\right)$. For any $x \in W$, we have

$$
\zeta_{p^{N}}^{-(\llcorner\circ \lambda)(x)}=\lambda^{\prime}(x)=\frac{\varphi_{\mathrm{I}^{\prime}}\left(\sqrt[p^{N}]{x}\right)}{\sqrt[p^{N}]{x}} \equiv \frac{\left(\sqrt[p^{N}]{x}\right)^{N\left(\mathrm{r}^{\prime}\right)}}{\sqrt[p^{N}]{x}}=x^{\frac{N\left(\mathrm{r}^{\prime}\right)-1}{p^{N}}}
$$

where the congruence is $\bmod \mathfrak{l}_{K\left(\mu_{\left.p^{n}\right)}^{\prime}\right.}$. Let $\phi_{K_{\mathfrak{l}_{K}^{\prime}}}: K_{\mathfrak{l}_{K}^{\prime}}^{\times} \longrightarrow G_{\mathfrak{l}^{\prime}} \otimes \mathbf{Z} / p^{N}$ be the reciprocity map, and $\operatorname{Kum}_{(N)}$ be the map defined in the proof of Lemma 2.2. Then by (2.1) we have

$$
\left(\operatorname{Kum}_{(N)} \circ \phi_{K_{\mathfrak{l}_{K}^{\prime}}}(x)\right) \equiv x^{-\frac{N\left(\mathfrak{l}^{\prime}\right)-1}{p^{N}}} \equiv \zeta_{p^{N}}^{(\llcorner 0 \lambda)(x)}\left(\bmod \mathfrak{l}_{K\left(\mu_{p^{n}}\right)}^{\prime}\right) .
$$

Since $\operatorname{Kum}_{(N)}\left(\sigma_{\mathfrak{l}^{\prime}}\right)=\zeta_{p^{N}}$, we have

$$
\phi_{K_{\mathrm{l}_{K}^{\prime}}}(x)=\sigma_{\mathfrak{l}^{\prime}}^{(\iota \circ \lambda)(x)}=(\iota \circ \lambda)(x) \otimes \sigma_{\mathfrak{l}^{\prime}} .
$$

Since $\left(\iota \circ \bar{\phi}_{\mathfrak{l}^{\prime}}\right)(x) \otimes \sigma_{\mathfrak{l}^{\prime}}=\phi_{K_{\mathfrak{l}_{K}^{\prime}}}(x)$ and $\mathcal{I}: f \mapsto \iota \circ f$ is an isomorphism, it follows that

$$
\bar{\phi}_{\mathbf{l}^{\prime}}(x)=\lambda(x) \text {. }
$$

This completes the proof of Lemma 9.1.

9.2. In this subsection, we prove Theorem 1.1 .

Step 1. (Preliminary argument on a minor of a relation matrix of $X_{K_{\infty}}^{\chi}$ )

By Lemma 8.1 (1), there is an exact sequence

$$
0 \longrightarrow \Lambda^{n} \stackrel{f}{\longrightarrow} \Lambda^{n} \stackrel{g}{\longrightarrow} X_{K_{\infty}}^{\chi} \longrightarrow 0 .
$$

Let $A$ be the matrix corresponding to $f$. Consider the matrix $A_{i}$ which is obtained from $A$ by eliminating the $n_{1}$-th row,..., the $n_{i}$-th row and the $m_{1}$-th column,..., the $m_{i}$-th column $\left(A_{i}\right.$ is an $(n-i) \times(n-i)$ matrix). Our aim is to prove that $\operatorname{det} A_{i}$ is in $\Theta_{i, K_{\infty}}^{(\delta), \chi}$.

We put $A_{0}=A$. By the main conjecture proved by Wiles (Theorem 8.6), we know $\left(\operatorname{det} A_{0}\right)=$ $\left(\theta_{K_{\infty}}^{\chi}\right)$. Thus, for $i=0, \Theta_{0, K_{\infty}}^{(\delta), \chi}=\left(\operatorname{det} A_{0}\right)$ holds. In order to make our argument simple, we take the above exact sequence (9.1) such that $\operatorname{det} A_{0}=\theta_{K}^{\chi}$. We also note that $\operatorname{det} A_{0} \neq 0$. Suppose $i \geq 1$ in the following. We will prove $\operatorname{det} A_{i} \in \Theta_{i, K_{\infty}}^{(\delta), \chi}$ by induction on $i$. First of all, since $0 \in \Theta_{i, K_{\infty}}^{\chi}$ is clear, we may assume $\operatorname{det} A_{i} \neq 0$. Furthermore, by changing the order of $m_{1}, \ldots, m_{i}$ if it is needed, we may assume $\operatorname{det} A_{r} \neq 0$ for all $r$ such that $0 \leq r \leq i$. In fact, let $B$ be the $(n-i+1) \times n$ matrix obtained from $A$ by eliminating the $n_{1}$-th row, $\ldots$, and the $n_{i-1}$-th row. For $l$ such that $1 \leq l \leq i$, we denote by $B_{l}$ the matrix obtained from $B$ by eliminating the $m_{j}$ th columns for all $j$ such that $1 \leq j \leq i$ and $j \neq l$. If $\operatorname{det} B_{l}=0$ for all $l(1 \leq l \leq i)$, the rank of $B_{l}$ is equal to the rank of $A_{i}$ which is $n-i$, so $\operatorname{rank} B=n-i$. This shows that $\operatorname{rank} A \leq n-i+i-1=n-1$, which implies $\operatorname{det} A=0$, and we get a contradiction. Therefore, 
one of $\operatorname{det} B_{l}$ is non-zero. Replacing $m_{i}$ with $m_{l}$, we get $\operatorname{det} A_{i-1}=\operatorname{det} B_{l} \neq 0$. Proceeding in this way, we can take $A_{r}$ such that $\operatorname{det} A_{r} \neq 0$ for all $r$ such that $0 \leq r \leq i$.

Step 2. (Definition of a homomorphism $\beta_{r}$ )

Taking $m$ sufficiently large, we may assume that all primes of $k$ above $p$ are ramified in $K_{0, m}$, and all primes of $K_{0, m}$ above $p$ are totally ramified in $K_{\infty}$. We take positive integers $N_{m}$ such that $N_{m} \rightarrow \infty$ as $m \rightarrow \infty$. To simplify the notation, we put $K=K_{0, m}, N=N_{m}$. Note that $K$ satisfies the conditions of $\S 3.1$ including $(*)$, and we apply the results in $\S 1-\S 8$ for $K$ and $N$.

Put $R_{K}=O_{\chi}[\Gamma(K / k)]=O_{\chi}\left[\operatorname{Gal}\left(K / K_{0}\right)\right]$, and denote by $\gamma_{m}$ a generator of $\operatorname{Gal}\left(K_{\infty} / K\right)=$ $\operatorname{Gal}\left(K_{\infty} / K_{0, m}\right)$. Since $\left(X_{K_{\infty}}^{\chi}\right)_{\operatorname{Gal}\left(K_{\infty} / K\right)} \simeq A_{K}^{\chi}$ is bijective by Lemma 8.9, it is finite. Therefore, $\gamma_{m}-1$ is prime to $\operatorname{char}\left(X_{K_{\infty}}^{\chi}\right)$, and $\operatorname{Gal}\left(K_{\infty} / K\right)$-invariant $\left(X_{K_{\infty}}^{\chi}\right)^{\operatorname{Gal}\left(K_{\infty} / K\right)}$ vanishes. Hence, taking $\operatorname{Gal}\left(K_{\infty} / K\right)$-coinvariants of the exact sequence $(9.1)$, by Lemma 8.9 we obtain an exact sequence

$$
0 \longrightarrow R_{K}^{n} \stackrel{\bar{f}}{\longrightarrow} R_{K}^{n} \stackrel{\bar{g}}{\longrightarrow} A_{K}^{\chi} \longrightarrow 0
$$

where $\bar{f}$ corresponds to the matrix $A \bmod \gamma_{m}-1$.

Let $\left(\mathbf{e}_{r}\right)_{1 \leq r \leq n}$ be the standard basis of $\Lambda^{n}$ in the exact sequence (9.1), and define $\mathbf{c}_{1}=$ $g\left(\mathbf{e}_{1}\right), \ldots, \mathbf{c}_{n}=g\left(\mathbf{e}_{n}\right)$ which are generators of $X_{K}^{\chi}$ as a $\Lambda$-module. We denote by $\mathbf{c}_{r}^{(m)}$ the image of $\mathbf{c}_{r}$ in $A_{K}^{\chi}$. The image of $\mathbf{e}_{r}$ in $R_{K}^{n}$ will be denoted by the same notation $\mathbf{e}_{r}$. Hence we have $\bar{g}\left(\mathbf{e}_{r}\right)=\mathbf{c}_{r}^{(m)} \in A_{K}^{\chi}$ for all $r$. Recall that we defined the set $\mathcal{S}_{[i-1]}(K)$ in $\S 4.2$. We define

$$
Q_{r}=\left\{\mathfrak{l} \in \mathcal{S}_{[i-1]}(K) \mid\left[\mathfrak{l}_{K}\right]^{\chi}=\mathbf{c}_{r}^{(m)}\right\}
$$

for each $r$ where $\left[\mathfrak{l}_{K}\right]^{\chi}$ is the class of $\mathfrak{l}_{K}$ in $A_{K}^{\chi}$. By the Chebotarev density theorem, $Q_{r}$ is an infinite set. We define $Q=\bigcup_{1 \leq r \leq n} Q_{r}$. Let $Q_{K}$ be the set of primes of $K$ above $Q$, and $\mathcal{D}=\bigoplus_{\rho \in Q_{K}} \mathbf{Z} \cdot \rho$ be the subgroup of $\operatorname{Div}_{K}$ consisting of all divisors whose supports are in $Q_{K}$. We have a natural surjective homomorphism

$$
\alpha:\left(\mathcal{D} \otimes \mathbf{Z}_{p}\right)^{\chi} \longrightarrow R_{K}^{n}
$$

defined by $\left[\mathfrak{l}_{K}\right]^{\chi} \mapsto \mathbf{e}_{r}$ for each $\mathfrak{l} \in Q_{r}$ and each $r$ with $1 \leq r \leq n$.

Let $\mathcal{K}$ denote the preimage of $\left(\mathcal{D} \otimes \mathbf{Z}_{p}\right)^{\chi}$ under the map $\left(K^{\times} \otimes \mathbf{Z}_{p}\right)^{\chi} \stackrel{\text { div }}{\longrightarrow}\left(\operatorname{Div}_{K} \otimes \mathbf{Z}_{p}\right)^{\chi}$. The exact sequence in Lemma 3.1 yields an exact sequence

$$
0 \longrightarrow \mathcal{K} \stackrel{\operatorname{div}}{\longrightarrow}\left(\mathcal{D} \otimes \mathbf{Z}_{p}\right)^{\chi} \longrightarrow A_{K}^{\chi} \longrightarrow 0 .
$$

The homomorphism $\alpha$ induces a surjective homomorphism $\beta: \mathcal{K} \longrightarrow R_{K}^{n}$ such that the diagram of exact sequences

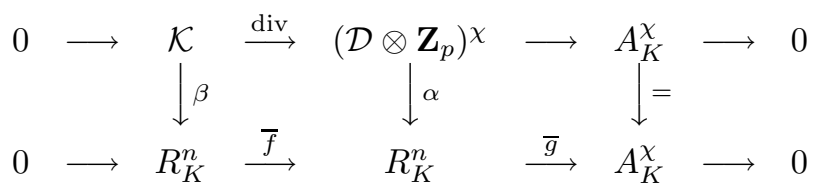

commutes. We define

$$
\beta_{r}=\operatorname{pr}_{r} \circ \beta: \mathcal{K} \stackrel{\beta}{\longrightarrow} R_{K}^{n} \stackrel{\operatorname{pr}_{r}}{\longrightarrow} R_{K}
$$

to be the composition of $\beta$ with the $r$-th projection.

Taking $\bmod p^{N}$ of the natural homomorphism $\mathcal{K} \longrightarrow\left(K^{\times} \otimes \mathbf{Z}_{p}\right)^{\chi}$, we consider

$$
\mathcal{K} / p^{N} \longrightarrow\left(K^{\times} / p^{N}\right)^{\chi}
$$

This is injective, and the image coincides with the preimage of $\left(\mathcal{D} / p^{N}\right)^{\chi}$ under the map $\left(K^{\times} / p^{N}\right)^{\chi} \stackrel{\text { div }}{\longrightarrow}\left(\operatorname{Div}_{K} / p^{N}\right)^{\chi}$. These properties can be checked by diagram chasing of the 
commutative diagram of exact sequences

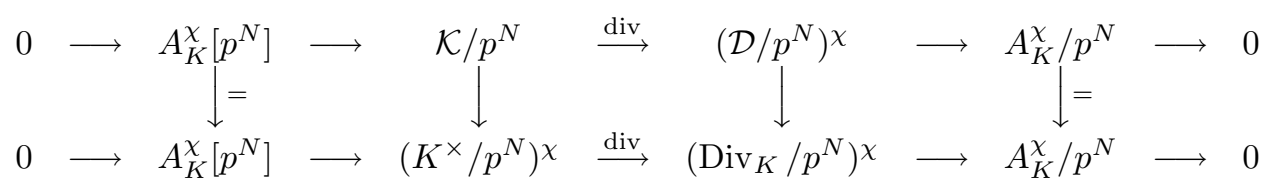

where the map $\left(\mathcal{D} / p^{N}\right)^{\chi} \longrightarrow\left(\operatorname{Div}_{K} / p^{N}\right)^{\chi}$ is injective. Using the above map $\mathcal{K} / p^{N} \longrightarrow$ $\left(K^{\times} / p^{N}\right)^{\chi}$, we identify $\mathcal{K} / p^{N}$ with the preimage of $\left(\mathcal{D} / p^{N}\right)^{\chi}$ under the map div.

For each $r$ with $1 \leq r \leq n$, we consider $\beta_{r} \bmod p^{N}: \mathcal{K} / p^{N} \longrightarrow R_{K} / p^{N}$ which we denote simply by $\beta_{r}$.

Step 3. (Definition of $x_{\mathfrak{n}, \mathfrak{r}}$ and a key Lemma 9.2)

For any $\mathfrak{n} \in \mathcal{N}_{[i-1]}(K)$ whose prime divisors are all in $Q, Q_{\mathfrak{n}, K}$ denotes the set of all prime divisors of $K$ dividing $\mathfrak{n}$. We define $\mathcal{D}_{\mathfrak{n}}=\bigoplus_{\rho \in Q_{\mathfrak{n}, K}} \mathbf{Z} \cdot \rho$ which is a subgroup of $\mathcal{D}$, and $\mathcal{K}_{\mathfrak{n}, N}$ to be the preimage of $\left(\mathcal{D}_{\mathfrak{n}} / p^{N}\right)^{\chi}$ under the map div $: \mathcal{K} / p^{N} \longrightarrow\left(\mathcal{D} / p^{N}\right)^{\chi}$. Note that $\mathcal{K}_{\mathfrak{n}, N}$ is a finite submodule of $\left(K^{\times} / p^{N}\right)^{\chi}$.

Recall that we are studying $A_{i}$ which is the matrix obtained from $A$ by eliminating the $n_{1}$-th row,$\ldots$, the $n_{i}$-th row and the $m_{1}$-th column,..., the $m_{i}$-th column. We choose $n_{i+1}, \ldots, n_{n}$ such that $\left\{n_{1}, \ldots, n_{n}\right\}=\{1, \ldots, n\}$. We take $\mathfrak{l}_{r} \in Q_{n_{r}}$ for each $r$ with $1 \leq r \leq n$, and fix them. Put $\mathfrak{L}=\mathfrak{l}_{1} \cdot \ldots \cdot \mathfrak{l}_{n}$. In the case $i=1$, we put $\mathfrak{n}=\mathfrak{n}_{1}=1$ and $\mathfrak{l}=\mathfrak{l}_{1}$. Suppose $i \geq 2$. We consider $\mathcal{K}_{\mathfrak{L}, N}$ and

$$
\beta_{m_{1}}: \mathcal{K}_{\mathfrak{L}, N} \longrightarrow R_{K} / p^{N} \text {. }
$$

Applying Lemma 9.1, we can take $\mathfrak{r}_{2} \in \mathcal{S}(K(\mathfrak{L}))$ such that $\mathfrak{r}_{2} \in Q_{n_{2}}, \mathfrak{r}_{2} \neq \mathfrak{l}_{2}$, and $\beta_{m_{1}}(x)=$ $\bar{\phi}_{\mathfrak{r}_{2}}(x)$ for all $x \in \mathcal{K}_{\mathfrak{L}, N}$. For any $r$ such that $2<r \leq i+1$, we take $\mathfrak{r}_{r}$ by induction on $r$. Put $\mathfrak{n}_{r-1}=\mathfrak{r}_{2} \cdot \ldots \cdot \mathfrak{r}_{r-1}$. We consider

$$
\beta_{m_{r-1}}: \mathcal{K}_{\mathfrak{L} \mathfrak{n}_{r-1}, N} \longrightarrow R_{K} / p^{N} .
$$

By induction on $r$, using Lemma 9.1, we take $\mathfrak{r}_{r} \in \mathcal{S}\left(K\left(\mathfrak{L} \mathfrak{n}_{r-1}\right)\right)$ such that

(I) $\mathfrak{r}_{r} \in Q_{n_{r}}$ and $\mathfrak{r}_{r} \neq \mathfrak{l}_{r}$,

(II) for the $b_{r} \in\left(K^{\times} \otimes \mathbf{Z}_{p}\right)^{\chi}$ such that $\operatorname{div}\left(b_{r}\right)=\left(\mathfrak{r}_{r, K}-\mathfrak{l}_{r, K}\right)^{\chi}, \phi_{\mathfrak{r}_{s}}\left(b_{r}\right)=0$ holds for any $s$ such that $2 \leq s<r$, and

(III) $\beta_{m_{r-1}}(\bar{x})=\bar{\phi}_{\mathfrak{r}_{r}}(x)$ for all $x \in \mathcal{K}_{\mathfrak{L} \mathfrak{n}_{r-1}, N}$

Thus, we have taken $\mathfrak{r}_{2}, \ldots, \mathfrak{r}_{i+1}$. (Note that $\mathfrak{r}_{1}$ is not defined.)

In the case $i \geq 2$, put $\mathfrak{l}=\mathfrak{l}_{1}$, and $\mathfrak{n}=\mathfrak{n}_{i}=\mathfrak{r}_{2} \cdot \ldots \cdot \mathfrak{r}_{i}$. In $\S 6$, we defined the element $x_{\mathfrak{n}, \mathfrak{l}}$ which is determined if $a_{\mathfrak{r}}$ is given for each $\mathfrak{r}$ dividing $\mathfrak{n}$. For each $\mathfrak{r}_{r}$ with $2 \leq r \leq i$, we take

$$
a_{\mathfrak{r}_{r}}=\phi_{\mathfrak{r}_{r}}\left(b_{r}\right)
$$

to define $x_{\mathfrak{n}, \mathfrak{l}}$. In the case $i=1, x_{\mathfrak{n}, \mathfrak{l}}=x_{\mathfrak{n}_{1}, \mathfrak{l}}=x_{1, \mathfrak{l}}=\kappa_{1, \mathfrak{l}}=g_{\mathfrak{l}}^{K}$. Since $\kappa_{\frac{\mathfrak{n}}{\mathfrak{d}}, \mathfrak{l}}$ 's are all in $\mathcal{K} / p^{N}$, $x_{\mathfrak{n}, \mathfrak{l}}$ is in $\mathcal{K} / p^{N}$.

Lemma 9.2. (i) For $r$ such that $2 \leq r \leq i$, let $\beta_{m_{r-1}}$ be the map defined in Step 2. Then we have

$$
\beta_{m_{r-1}}\left(x_{\mathfrak{n}, \mathfrak{l}}\right)=0
$$

for any $r$ such that $2 \leq r \leq i$.

(ii) Let $\alpha_{j}=\operatorname{pr}_{j} \circ \alpha:\left(\mathcal{D} / p^{N}\right)^{\chi} \stackrel{\alpha}{\longrightarrow}\left(R_{K} / p^{N}\right)^{n} \stackrel{\mathrm{pr}_{j}}{\longrightarrow} R_{K} / p^{N}$ be the composition of $\alpha$ with the $j$-th projection. Then we have

$$
\alpha_{j}\left(\operatorname{div}\left(x_{\mathfrak{n}, \mathfrak{l}}\right)\right)=0
$$

for any $j$ such that $j \neq n_{1}, \ldots, n_{i}$. 
Proof of Lemma 9.2. Since $x_{\mathfrak{n}, \mathfrak{l}}$ is a unit outside $\mathfrak{n} \mathfrak{l}$ (Proposition $6.3(0)$ ), (ii) is immediate from the definition of $x_{\mathfrak{n}, \mathfrak{l}}$ (and the above property (I)). We will prove (i). For any $r$ such that $2 \leq r \leq i$, let $b_{r} \in\left(K^{\times} \otimes \mathbf{Z}_{p}\right)^{\chi}$ be the element such that $\operatorname{div}\left(b_{r}\right)=\left(\mathfrak{r}_{r, K}-\mathfrak{l}_{r, K}\right)^{\chi}$. By the definition of $\alpha$, we have $\alpha\left(\operatorname{div}\left(b_{r}\right)\right)=0$, hence we know from the definition of $\beta$ that $\beta\left(b_{r}\right)=0$ for any $r$ such that $2 \leq r \leq i$. Put

$$
x=x_{\mathfrak{n}, l} b_{r}^{-\bar{\phi}_{\mathbf{v}_{r}}\left(x_{\frac{\mathfrak{n}}{\mathfrak{r}_{r}}, \mathrm{l}}\right)} \ldots b_{i}^{-\bar{\phi}_{\mathbf{r}_{i}}\left(x_{\frac{\mathfrak{n}}{\mathfrak{r}_{i}}, \mathfrak{l}}\right)} .
$$

It follows from $\beta\left(b_{r}\right)=\ldots=\beta\left(b_{i}\right)=0$ that

$$
\beta_{m_{r-1}}\left(x_{\mathfrak{n}, \mathfrak{l}}\right)=\beta_{m_{r-1}}(x) .
$$

By Proposition 6.3 (1), we have $\operatorname{div}_{\mathfrak{r}_{s}}(x)=\bar{\phi}_{\mathfrak{r}_{s}}\left(x_{\frac{\mathfrak{n}}{\mathrm{r}_{\mathrm{s}}}, \mathfrak{l}}\right)-\bar{\phi}_{\mathbf{r}_{s}}\left(x_{\frac{\mathfrak{n}}{\mathrm{r}_{s}}, \mathfrak{l}}\right)=0$ for any $s$ such that $r \leq$ $s \leq i$. This shows that $\operatorname{div}(x) \in\left(\mathcal{D}_{\mathfrak{L} \mathfrak{n}_{r-1}} / p^{N}\right)^{\chi}$, which implies $x \in \mathcal{K}_{\mathfrak{L} \mathfrak{n}_{r-1}, N}$. Hence, applying the above property (III), we obtain

$$
\beta_{m_{r-1}}(x)=\bar{\phi}_{\mathfrak{r}_{r}}(x) .
$$

By the above property (II), we have $\bar{\phi}_{\mathfrak{r}_{r}}\left(b_{r+1}\right)=\ldots=\bar{\phi}_{\mathfrak{r}_{r}}\left(b_{i}\right)=0$. Therefore, we get

$$
\bar{\phi}_{\mathbf{r}_{r}}(x)=\bar{\phi}_{\mathfrak{r}_{r}}\left(x_{\mathfrak{n}, l} b_{r}^{-\overline{\mathbf{x}}_{\mathfrak{r}_{r}}\left(x_{\frac{\mathfrak{n}}{\mathfrak{r}_{r}, \mathrm{l}}}\right)}\right) .
$$

Now, using Proposition 6.3 (2), we have

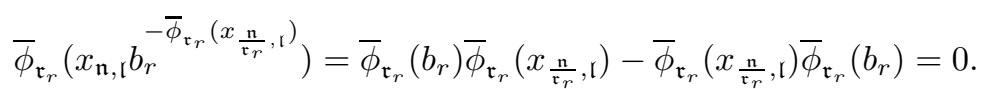

Therefore, we have obtained $\beta_{m_{r-1}}\left(x_{\mathfrak{n}, \mathfrak{l}}\right)=0$, which completes the proof of Lemma 9.2.

Step 4. (Approximation of the minor $\operatorname{det} A_{i}$ )

We go back to the proof of Theorem 1.1. Since $x_{\mathfrak{n}, \mathfrak{l}}$ is in $\mathcal{K}_{\mathfrak{L} \mathfrak{n}_{i}, N}$, we note that we also have

$$
\beta_{m_{i}}\left(x_{\mathfrak{n}, \mathfrak{l}}\right)=\bar{\phi}_{\mathfrak{r}_{i+1}}\left(x_{\mathfrak{n}, \mathfrak{l}}\right)
$$

by the property (III).

Put $\mathbf{x}=\beta\left(x_{\mathfrak{n}, \mathfrak{l}}\right) \in\left(R_{K} / p^{N}\right)^{n}$ and $\mathbf{y}=\alpha\left(\operatorname{div}\left(x_{\mathfrak{n}, \mathfrak{l}}\right)\right) \in\left(R_{K} / p^{N}\right)^{n}$, which we regard as column vectors. Since $R_{K} / p^{N}=\Lambda /\left(\gamma_{m}-1, p^{N}\right)$, we have

$$
A \mathbf{x} \equiv \mathbf{y} \quad\left(\bmod \left(\gamma_{m}-1, p^{N}\right)\right) .
$$

Let $\mathbf{x}^{\prime} \in\left(R_{K} / p^{N}\right)^{n-i+1}$ be the vector obtained from $\mathbf{x}$ by eliminating the $m_{1}$-th row,,.., and the $m_{i-1}$-th row, and $\mathbf{y}^{\prime} \in\left(R_{K} / p^{N}\right)^{n-i+1}$ the vector obtained from $\mathbf{y}$ by eliminating the $n_{1}$-th row,,$\ldots$, and the $n_{i-1}$-th row. It follows from Lemma 9.2 (i) that the $m_{r}$-th row of $\mathbf{x}$ is zero in $R_{K} / p^{N}$ for all $r$ such that $1 \leq r \leq i-1$. Therefore, we have

$$
A_{i-1} \mathbf{x}^{\prime} \equiv \mathbf{y}^{\prime} \quad\left(\bmod \left(\gamma_{m}-1, p^{N}\right)\right) .
$$

If $i \geq 2$, the $n_{i}$-th component of $\mathbf{y}$ is $\bar{\phi}_{\mathfrak{r}_{i}}\left(x_{\frac{\mathfrak{n}}{\mathfrak{r}_{i}}, l}\right)=\bar{\phi}_{\mathfrak{r}_{i}}\left(x_{\mathfrak{n}_{i-1}, l}\right)$ by Proposition 6.3 (1). Hence, if the $n_{i}^{\prime}$-th component of $\mathbf{y}^{\prime}$ is the $n_{i}$-th component of $\mathbf{y}$, by Lemma 9.2 (ii) we have

$$
\mathbf{y}^{\prime}=\bar{\phi}_{\mathfrak{r}_{i}}\left(x_{\mathfrak{n}_{i-1}, l}\right) \mathbf{e}_{n_{i}^{\prime}}
$$

where $\mathbf{e}_{n_{i}^{\prime}}$ denotes the $n_{i}^{\prime}$-th standard basis vector of $\left(R_{K} / p^{N}\right)^{n-i+1}$. We saw $\beta_{m_{i}}\left(x_{\mathfrak{n}, \mathfrak{l}}\right)=$ $\bar{\phi}_{\mathfrak{r}_{i+1}}\left(x_{\mathfrak{n}, \mathfrak{l}}\right)$ above. Therefore, the $m_{i}$-th component of $\mathbf{x}$ is $\bar{\phi}_{\mathbf{r}_{i+1}}\left(x_{\mathfrak{n}, \mathfrak{l}}\right)$. We suppose that the $m_{i}^{\prime}$-th component of $\mathbf{x}^{\prime}$ is the $m_{i}$-th component of $\mathbf{x}$. Let $\operatorname{Adj}\left(A_{i-1}\right)$ be the matrix of cofactors (namely, the $(s, t)$ entry of $\operatorname{Adj}\left(A_{i-1}\right)$ is $(-1)^{s+t} \operatorname{det} P_{t s}$ where $P_{t s}$ is the matrix obtained by eliminating the $t$-th row and the $s$-th column of $\left.A_{i-1}\right)$. Multiplying both sides of $A_{i-1} \mathbf{x}^{\prime} \equiv \bar{\phi}_{\mathfrak{r}_{i}}\left(x_{\mathfrak{n}_{i-1}, \mathfrak{l}}\right) \mathbf{e}_{n_{i}^{\prime}}$ by $\operatorname{Adj}\left(A_{i-1}\right)$ on the left, we get

$$
\left(\operatorname{det} A_{i-1}\right) \mathbf{x}^{\prime} \equiv \bar{\phi}_{\mathfrak{r}_{i}}\left(x_{\mathfrak{n}_{i-1}, \mathrm{l}}\right) \operatorname{Adj}\left(A_{i-1}\right) \mathbf{e}_{n_{i}^{\prime}} .
$$


Hence we obtain

$$
\left(\operatorname{det} A_{i-1}\right) \bar{\phi}_{\mathfrak{r}_{i+1}}\left(x_{\mathfrak{n}, \mathfrak{l}}\right) \equiv(-1)^{n_{i}^{\prime}+m_{i}^{\prime}}\left(\operatorname{det} A_{i}\right) \bar{\phi}_{\mathfrak{r}_{i}}\left(x_{\mathfrak{n}_{i-1}, \mathfrak{l}}\right) \quad\left(\bmod \left(\gamma_{m}-1, p^{N}\right)\right) .
$$

Recall that $\mathfrak{n}=\mathfrak{n}_{i}$. We are not concerned in the sign problem, and write the above equation as

$$
\left(\operatorname{det} A_{i-1}\right) \bar{\phi}_{\mathfrak{r}_{i+1}}\left(x_{\mathfrak{n}_{i}, \mathfrak{l}}\right) \equiv \pm\left(\operatorname{det} A_{i}\right) \bar{\phi}_{\mathfrak{r}_{i}}\left(x_{\mathfrak{n}_{i-1}, \mathfrak{l}}\right) \quad\left(\bmod \left(\gamma_{m}-1, p^{N}\right)\right) \text {. }
$$

If $i=1$, since $x_{1, \mathfrak{l}}=g_{\mathfrak{l}}^{K}$, the $n_{1}$-th component of $\mathbf{y}$ is $\theta_{K}^{\chi}$, and $\mathbf{y}=\theta_{K}^{\chi} \mathbf{e}_{n_{1}}$. Therefore, by the same method as above, we obtain

$$
\left(\operatorname{det} A_{0}\right) \bar{\phi}_{\mathfrak{r}_{2}}\left(x_{\mathfrak{n}_{1}, \mathfrak{l}}\right) \equiv(-1)^{n_{1}+m_{1}}\left(\operatorname{det} A_{1}\right) \theta_{K}^{\chi} \quad\left(\bmod \left(\gamma_{m}-1, p^{N}\right)\right) .
$$

In order to clarify that we are working over $K=K_{0, m}$, we write $\bar{\phi}_{\mathfrak{r}_{i+1}}\left(x_{\mathfrak{n}_{i}, \mathfrak{l}}\right)_{m}$ for $\bar{\phi}_{\mathfrak{r}_{i+1}}\left(x_{\mathfrak{n}_{i}, \mathfrak{l}}\right)$.

LEMMA 9.3. For $i \geq 1$, the limit of $\bar{\phi}_{\mathfrak{r}_{i+1}}\left(x_{\mathfrak{n}_{i}, \mathfrak{l}}\right)_{m}$ exists in $\Lambda$ as $m$ goes to $\infty$. (Namely, if $\bar{\phi}_{\mathfrak{r}_{i+1}}\left(x_{\mathfrak{n}_{i}, \mathfrak{l}}\right)_{m}^{\prime} \in \Lambda$ is a lifting of $\bar{\phi}_{\mathfrak{r}_{i+1}}\left(x_{\mathfrak{n}_{i}, \mathfrak{l}}\right)_{m} \in \Lambda /\left(p^{N_{m}}, \gamma_{m}-1\right), \lim _{m \rightarrow \infty} \bar{\phi}_{\mathfrak{r}_{i+1}}\left(x_{\mathfrak{n}_{i}, \mathfrak{l}}\right)_{m}^{\prime}$ exists. $) W e$ denote the limit by $\lim _{m \rightarrow \infty} \bar{\phi}_{\mathfrak{r}_{i+1}}\left(x_{\mathfrak{n}_{i}, \mathfrak{l}}\right)_{m}$. We also have

$$
\lim _{m \rightarrow \infty} \bar{\phi}_{\mathfrak{r}_{i+1}}\left(x_{\mathfrak{n}_{i}, \mathfrak{l}}\right)_{m}= \pm \operatorname{det} A_{i} \in \Lambda
$$

Proof of Lemma 9.3. Recall that we took $A_{0}$ such that $\operatorname{det} A_{0}=\theta_{K_{\infty}}^{\chi}$ in the beginning of this subsection. We have $\lim _{m \rightarrow \infty} \theta_{K_{0, m}}^{\chi}=\theta_{K_{\infty}}^{\chi}=\operatorname{det} A_{0}$. Hence the above congruence (9.4) on $\bar{\phi}_{\mathfrak{r}_{2}}\left(x_{\mathfrak{n}_{1}, \mathfrak{l}}\right)$ implies that the limit of $\bar{\phi}_{\mathfrak{r}_{2}}\left(x_{\mathfrak{n}_{1}, \mathfrak{l}}\right)_{m}$ exists, and

$$
\lim _{m \rightarrow \infty} \bar{\phi}_{\mathfrak{r}_{2}}\left(x_{\mathfrak{n}_{1}, \mathfrak{l}}\right)_{m}= \pm \operatorname{det} A_{1}
$$

because $\operatorname{det} A_{0} \neq 0$. For general $i \geq 2$, by the same method as for $i=1$, using (9.3) for $\bar{\phi}_{\mathfrak{r}_{i+1}}\left(x_{\mathfrak{n}_{i}, \mathfrak{l}}\right)$ and induction on $i$, we get

$$
\operatorname{det} A_{i-1} \lim _{m \rightarrow \infty} \bar{\phi}_{\mathfrak{r}_{i+1}}\left(x_{\mathfrak{n}_{i}, \mathfrak{l}}\right)_{m}= \pm \operatorname{det} A_{i} \operatorname{det} A_{i-1}
$$

(we note that the sign does not depend on $m$ ). Recall that we took $A_{r}$ such that $\operatorname{det} A_{r} \neq 0$ for any $r$ with $1 \leq r \leq i$. Therefore, the limit of $\bar{\phi}_{\mathfrak{r}_{i+1}}\left(x_{\mathfrak{n}_{i}, \mathfrak{l}}\right)_{m}$ exists, and we get

$$
\lim _{m \rightarrow \infty} \bar{\phi}_{\mathfrak{r}_{i+1}}\left(x_{\mathfrak{n}_{i}, \mathfrak{l}}\right)_{m}= \pm \operatorname{det} A_{i} \text {. }
$$

Step 5. (Final step of the proof of Theorem 1.1)

We now prove Theorem 1.1. Put $I_{m}=\left(p^{N_{m}}, \gamma_{m}-1\right)$, and $\xi_{m}=\bar{\phi}_{\mathfrak{r}_{i+1}}\left(x_{\mathfrak{n}_{i}, \mathrm{l}}\right)_{m} \in \Lambda / I_{m}$. Note that $\left(\xi_{m}\right)_{m}$ might not be a projective system (namely, $\left(\xi_{m}\right)_{m} \in \lim \Lambda / I_{m}$ might not hold). Since $\xi_{m}$ converges, the image $\xi_{n, m}$ of $\xi_{n}$ for sufficiently large $n \gg \overleftarrow{m}$ under the natural map $\pi_{n, m}: \Lambda / I_{n} \longrightarrow \Lambda / I_{m}$ does not depend on the choice of $n$. We denote it by $\xi_{m}^{\prime}$. If the following Lemma 9.4 holds, applying it to $\xi_{n} \in \Lambda / I_{n}$, we have $\xi_{n} \in \Theta_{i, K_{0, n}}^{(\delta, N), \chi}$. Since $\pi_{n, m}\left(\Theta_{i, K_{0, n}}^{\left(\delta, N_{n}\right), \chi}\right) \subset$ $\Theta_{i, K_{0, m}}^{\left(\delta, N_{m}\right), \chi}, \xi_{m}^{\prime}=\xi_{n, m}$ is in $\Theta_{i, K_{0, m}}^{\left(\delta, N_{m}\right), \chi}$. By the construction of $\xi_{m}^{\prime},\left(\xi_{m}^{\prime}\right)_{m}$ becomes a projective system. Hence we obtain $\left(\xi_{m}^{\prime}\right)_{m} \in \underset{\leftarrow}{\lim } \Theta_{i, K_{0, m}}^{\left(\delta, N_{m}\right), \chi}=\Theta_{i, K_{\infty}}^{(\delta), \chi}$. This shows that

$$
\operatorname{det} A_{i}= \pm \lim _{m \rightarrow \infty} \xi_{m}= \pm \lim _{m \rightarrow \infty} \xi_{m}^{\prime} \in \Theta_{i, K_{\infty}}^{(\delta), \chi}
$$

Therefore, we have

$$
\operatorname{Fitt}_{i, \Lambda}\left(X_{K_{\infty}}^{\chi}\right) \subset \Theta_{i, K_{\infty}}^{(\delta), \chi}
$$


Combining the above inclusion with the inclusions $\Theta_{i, K_{\infty}}^{(\delta), \chi} \subset \Theta_{i, K_{\infty}}^{\chi} \subset \operatorname{Fitt}_{i, \Lambda}\left(X_{K_{\infty}}^{\chi}\right)$ in Corollary 8.12 , we get

$$
\operatorname{Fitt}_{i, \Lambda}\left(X_{K_{\infty}}^{\chi}\right)=\Theta_{i, K_{\infty}}^{(\delta), \chi}=\Theta_{i, K_{\infty}}^{\chi}
$$

Therefore, our final task is to prove

Lemma 9.4. For any $i \geq 1, \bar{\phi}_{\mathfrak{r}_{i+1}}\left(x_{\mathfrak{n}_{i}, \mathfrak{l}}\right)_{m}$ is in $\Theta_{i, K_{0, m}}^{\left(\delta, N_{m}\right), \chi}$.

Proof of Lemma 9.4. To simplify the notation, we again write $K=K_{0, m}, N=N_{m}, \mathfrak{n}=\mathfrak{n}_{i}$, and $\bar{\phi}_{\mathfrak{r}_{i+1}}\left(x_{\mathfrak{n}, \mathfrak{l}}\right)=\bar{\phi}_{\mathfrak{r}_{i+1}}\left(x_{\mathfrak{n}_{i}, \mathfrak{l}}\right)_{m}$. Applying Lemma 9.1 to the map $\bar{\phi}_{\mathfrak{r}_{i+1}}: \mathcal{K}_{\mathfrak{n} \mathfrak{l}, N} \longrightarrow R_{K} / p^{N}$, we can take $\mathfrak{l}^{\prime} \in Q_{n_{1}}$ which satisfies the properties stated before Lemma 6.2 , and

$$
\bar{\phi}_{\mathfrak{r}_{i+1}}(x)=\bar{\phi}_{\mathfrak{l}^{\prime}}(x)
$$

for any $x \in \mathcal{K}_{\mathfrak{n} \mathfrak{l}, N}$. In particular, we have

$$
\bar{\phi}_{\mathfrak{r}_{i+1}}\left(x_{\mathfrak{n}, \mathfrak{l}}\right)=\bar{\phi}_{\mathfrak{l}^{\prime}}\left(x_{\mathfrak{n}, \mathfrak{l}}\right) .
$$

Let $b \in\left(K^{\times} \otimes \mathbf{Z}_{p}\right)^{\chi}$ be the element such that $\operatorname{div}(b)=\left(\mathfrak{l}_{K}^{\prime}-\mathfrak{l}_{K}\right)^{\chi}$. By Proposition 6.3 (3), we have

$$
\bar{\phi}_{\mathfrak{r}_{i+1}}\left(x_{\mathfrak{n}, \mathfrak{l}}\right)=\bar{\phi}_{\mathfrak{l}^{\prime}}\left(x_{\mathfrak{n}, \mathfrak{l}}\right)=-\sum_{\mathfrak{d} \mid \mathfrak{n}} \bar{a}_{\mathfrak{d}}\left(\delta_{\frac{\mathfrak{n}}{\mathfrak{d}} \mathfrak{l}^{\prime}}+\delta_{\frac{\mathfrak{n}}{\mathfrak{d}}} \bar{\phi}_{\mathfrak{l}^{\prime}}(b)\right) .
$$

Since $\epsilon\left(\frac{\mathfrak{n}}{\mathfrak{d}}\right)<\epsilon\left(\frac{\mathfrak{n}}{\mathfrak{d}} \mathfrak{l}^{\prime}\right) \leq \epsilon\left(\mathfrak{n} \mathfrak{l}^{\prime}\right)=i$ for any $\mathfrak{d}(\epsilon(\mathfrak{n})$ is defined in the beginning of $\S 4.2)$, both $\delta_{\frac{\mathfrak{n}}{\mathfrak{d}}} \mathfrak{l}^{\prime}$ and $\delta_{\frac{\mathfrak{n}}{\mathfrak{d}}}$ are in $\Theta_{i, K}^{(\delta, N), \chi}$. Hence we get

$$
\bar{\phi}_{\mathfrak{r}_{i+1}}\left(x_{\mathfrak{n}, \mathfrak{l}}\right) \in \Theta_{i, K}^{(\delta, N), \chi} .
$$

This completes the proof of Lemma 9.4 and Theorem 1.1.

9.3. In this subsection, we prove Theorem 1.3 and Corollary 1.4 .

We first prove Theorem 1.3. Let $\pi_{m}: \Lambda \longrightarrow R_{K_{0, m}}$ be the natural map for any $m \geq 0$. By Lemma 8.9, we have $\pi_{m}\left(\operatorname{Fitt}_{i, \Lambda}\left(X_{K_{\infty}}^{\chi}\right)\right)=\operatorname{Fitt}_{i, R_{K_{0, m}}}\left(A_{K_{0, m}}^{\chi}\right)$. Since $\pi_{m}\left(\Theta_{i, K_{\infty}}^{(\delta), \chi}\right) \subset \Theta_{i, K_{0, m}}^{(\delta), \chi}$ by definition, using (9.5) in the proof of Theorem 1.1, we have

$$
\operatorname{Fitt}_{i, R_{K_{0}, m}}\left(A_{K_{0, m}}^{\chi}\right)=\pi_{m}\left(\operatorname{Fitt}_{i, \Lambda}\left(X_{K_{\infty}}^{\chi}\right)\right)=\pi_{m}\left(\Theta_{i, K_{\infty}}^{\chi}\right)=\pi_{m}\left(\Theta_{i, K_{\infty}}^{(\delta), \chi}\right) \subset \Theta_{i, K_{0, m}}^{(\delta), \chi} \subset \Theta_{i, K_{0, m}}^{\chi} .
$$

The last inclusion is (7.1) in $\S 7$. On the other hand, we have the other inclusion $\Theta_{i, K_{0, m}}^{\chi} \subset$ $\operatorname{Fitt}_{i, R_{K_{0}, m}}\left(A_{K_{0, m}}^{\chi}\right)$ by Theorem 8.11, so we get the equality $\operatorname{Fitt}_{i, R_{K_{0, m}}}\left(A_{K_{0, m}}^{\chi}\right)=\Theta_{i, K_{0, m}}^{(\delta), \chi}=$ $\Theta_{i, K_{0, m}}^{\chi}$.

Next, we prove Corollary 1.4. Since we have shown $\pi_{m}\left(\Theta_{i, K_{\infty}}^{\chi}\right)=\Theta_{i, K_{0, m}}^{\chi}$ above, $\Theta_{i}^{\chi \psi}$ is the image of $\Theta_{i, K_{\infty}}^{\chi}$ under the map $\Lambda \stackrel{\pi_{m}}{\longrightarrow} R_{K_{0, m}} \stackrel{\psi}{\longrightarrow} O_{\chi \psi}$. Therefore, Corollary 1.4 is an immediate consequence of Theorem 1.1 and Lemma 8.5.

9.4. Finally, we give two remarks in this subsection.

REMARK 9.5. We give some examples of numerical computation. Take $k=\mathbf{Q}, K_{0}=$ $\mathbf{Q}(\sqrt{-2437}), p=3$, and $\chi$ to be the character associated to $K_{0}$. Then all the assumptions of Theorem 1.1 are satisfied. We identify $\Lambda$ with $\mathbf{Z}_{p}[[T]]$, using the correspondence between $\gamma$ and $1+T$ where $\gamma$ is the generator of $\operatorname{Gal}\left(K_{\infty} / K_{0}\right)$ such that $\kappa(\gamma)=1+p$ where $\kappa$ is the cyclotomic character. It is easy to check that the $\lambda$-invariant of the $p$-adic $L$-function $\theta_{K_{\infty}}^{\chi}$ is 2 , and $A_{K_{0}}=(\mathbf{Z} / 3 \mathbf{Z})^{\oplus 2}$. We have $\operatorname{Fitt}_{1, \Lambda}\left(X_{K_{\infty}}\right) \neq \Lambda$ because $\operatorname{Fitt}_{1, \mathbf{Z}_{3}}\left(A_{K_{0}}\right)=(3) \neq \mathbf{Z}_{3}$. We 
regard $\theta_{K_{\infty}}^{\chi}=\theta_{K_{\infty}}^{\chi}(T)$ as an element of $\mathbf{Z}_{3}[[T]]$, then we have $\operatorname{ord}_{3}\left(\theta_{K_{\infty}}^{\chi}(0)\right)=2$. For a prime $\ell$ such that $\ell \equiv 1\left(\bmod p^{2}\right), K_{0}(\ell)$ denotes the maximal $p$-extension of $K_{0}$ in $K_{0}\left(\mu_{\ell}\right)$, and $G_{\ell}=\operatorname{Gal}\left(K_{0}(\ell) / K_{0}\right)$. We consider $\theta_{K_{0}(\ell)_{\infty}}^{\chi} \in \Lambda\left[G_{\ell}\right]$, and write

$$
\theta_{K_{0}(\ell)_{\infty}}^{\chi}=\delta_{0}^{(\ell)}+\delta_{1}^{(\ell)}\left(\sigma_{\ell}-1\right)+\delta_{2}^{(\ell)}\left(\sigma_{\ell}-1\right)^{2}+\ldots
$$

where $\delta_{i}^{(\ell)} \in \Lambda$ for $i \geq 0$. We compute the image $\overline{\delta_{1}^{(\ell)}}$ of $\delta_{1}^{(\ell)}$ in $\Lambda /\left(9, T^{2}\right)=\mathbf{Z}_{3}[T] /\left(9, T^{2}\right)$, and obtain

$$
\overline{\delta_{1}^{(19)}}=2 T+3 \text { and } \overline{\delta_{1}^{(37)}}=7 T \text {. }
$$

Hence Theorem 1.1 implies that $\operatorname{Fitt}_{1, \Lambda}\left(X_{K_{\infty}}\right) \bmod \left(9, T^{2}\right)$ contains $2 T+3$ and $7 T$. This shows that the image of $\operatorname{Fitt}_{1, \Lambda}\left(X_{K_{\infty}}\right)$ in $\Lambda /\left(p^{2}, T^{2}\right)$ contains $(3, T)$, which implies that $\operatorname{Fitt}_{1, \Lambda}\left(X_{K_{\infty}}\right) \supset(3, T)$. Since $\operatorname{Fitt}_{1, \Lambda}\left(X_{K_{\infty}}\right) \neq \Lambda$, it follows that

$$
\operatorname{Fitt}_{1, \Lambda}\left(X_{K_{\infty}}\right)=(3, T) \text {. }
$$

By Lemma 8.3, the information that $\operatorname{Fitt}_{0, \Lambda}\left(X_{K_{\infty}}\right)=\left(\theta_{K_{\infty}}^{\chi}\right), \operatorname{Fitt}_{1, \Lambda}\left(X_{K_{\infty}}\right)=(3, T)$, and $\operatorname{Fitt}_{2, \Lambda}\left(X_{K_{\infty}}\right)=\Lambda$ determines the isomorphism class of $X_{K_{\infty}}^{\chi}$ (Concerning relation matrices of $X_{K_{\infty}}^{\chi}$, see [9] Lemma 9.1).

For $K_{0}=\mathbf{Q}(\sqrt{-6226})$ (resp. $\mathbf{Q}(\sqrt{-6910})$ ) and $p=3$, the $\lambda$-invariant of $\theta_{K_{\infty}}^{\chi}$ is also 2 , and $A_{K_{0}}=(\mathbf{Z} / 3 \mathbf{Z})^{\oplus 2}$. We can also compute

$$
\begin{gathered}
\overline{\delta_{1}^{(19)}}=3 \text { and } \overline{\delta_{1}^{(37)}}=2 T+3 \\
\left(\text { resp. } \overline{\delta_{1}^{(19)}}=3 T+3 \text { and } \overline{\delta_{1}^{(37)}}=7 T\right) .
\end{gathered}
$$

Therefore, we get $\operatorname{Fitt}_{1, \Lambda}\left(X_{K_{\infty}}\right)=(3, T)$ in these two cases, too. Hence the isomorphism class is also determined by these data. In [7], Koike determined the isomorphism classes for many numerical examples, but in these two cases $(\mathbf{Q}(\sqrt{-6226})$ and $\mathbf{Q}(\sqrt{-6910}))$ the isomorphism classes were not determined by his method.

REMARK 9.6. Using the theory in this paper, we can compute in several cases not only the Fitting ideals but also the matrix corresponding to $\bar{f}$ in $(9.2)$ in $\S 9.2$. We will give a simple example.

Suppose that $K=K_{0, m}, \mathfrak{l}_{1} \mathfrak{l}_{2} \in \mathcal{N}_{[2]}(K)$ and $\mathfrak{l}_{2} \in \mathcal{S}\left(K\left(\mathfrak{l}_{1}\right)\right)$. We assume that $\delta_{\mathfrak{l}_{1} \mathfrak{l}_{2}}$ is a unit in $R_{K} / p^{N}$. (The numerical computation of $\delta_{\mathfrak{n}}$ is easy in general.) Then $\operatorname{Fitt}_{2, R_{K}}\left(A_{K}^{\chi}\right)=R_{K}$ by Theorem 1.3, and $A_{K}^{\chi}$ is generated by two elements over $R_{K}$ (cf. $\S 8.1$ ). Assume further that $A_{K}^{\chi}$ is generated by $\left[\mathfrak{l}_{1}\right]^{\chi}$ and $\left[\mathfrak{l}_{2}\right]^{\chi}$. Then $\mathcal{K}_{\mathfrak{l}_{1} \mathfrak{l}_{2}, N}$ is a free $R_{K} / p^{N}$-module of rank 2 , and $\kappa_{\mathfrak{l}_{1}, \mathfrak{l}_{2}}, \kappa_{\mathfrak{l}_{2}, \mathfrak{l}_{1}}$ is a basis of $\mathcal{K}_{\mathfrak{l}_{1} \mathfrak{l}_{2}, N}$. In fact, by Propositions 5.4 and 5.5, we have $\bar{\phi}_{\mathfrak{l}_{1}}\left(\kappa_{\mathfrak{l}_{1}, \mathfrak{l}_{2}}\right)=0, \bar{\phi}_{\mathfrak{l}_{2}}\left(\kappa_{\mathfrak{l}_{1}, \mathfrak{l}_{2}}\right)=\delta_{\mathfrak{l}_{1} \mathfrak{l}_{2}}$, $\bar{\phi}_{\mathfrak{l}_{1}}\left(\kappa_{\mathfrak{l}_{2}, \mathfrak{l}_{1}}\right)=\delta_{\mathfrak{l}_{1} \mathfrak{l}_{2}}$, and $\bar{\phi}_{\mathfrak{l}_{2}}\left(\kappa_{\mathfrak{l}_{2}, \mathfrak{l}_{1}}\right)=0$. This shows that $\bar{\phi}_{\mathfrak{l}_{1}} \oplus \bar{\phi}_{\mathfrak{l}_{2}}: \mathcal{K}_{\mathfrak{l}_{1} \mathfrak{l}_{2}, N} \longrightarrow\left(R_{K} / p^{N}\right)^{\oplus 2}$ is an isomorphism, and $\kappa_{\mathfrak{l}_{1}, \mathfrak{l}_{2}}, \kappa_{\mathfrak{l}_{2}, \mathfrak{l}_{1}}$ is a basis of $\mathcal{K}_{\mathfrak{l}_{1} \mathfrak{l}_{2}, N}$. Consider the exact sequence

$$
\mathcal{K}_{\mathfrak{l}_{1} \mathfrak{l}_{2}} / p^{N} \stackrel{\operatorname{div}}{\longrightarrow}\left(\mathcal{D}_{\mathfrak{l}_{1} \mathfrak{l}_{2}} / p^{N}\right)^{\chi} \longrightarrow A_{K}^{\chi} / p^{N} \longrightarrow 0
$$

Using the basis $\kappa_{\mathfrak{l}_{1}, \mathfrak{l}_{2}}, \kappa_{\mathfrak{l}_{2}, \mathfrak{l}_{1}}$, we can compute the relation matrix of $A_{K}^{\chi} / p^{N}$ to be

$$
\left(\begin{array}{cc}
\bar{\phi}_{\mathfrak{l}_{1}}\left(g_{\mathfrak{l}_{2}}^{K}\right) & \delta_{\mathfrak{l}_{2}} \\
\delta_{\mathfrak{l}_{1}} & \bar{\phi}_{\mathfrak{l}_{2}}\left(g_{\mathfrak{l}_{1}}^{K}\right)
\end{array}\right)
$$

by Proposition 4.2. Note that the entries of the matrix are numerically computable in principle if $k=\mathbf{Q}$. This is also an example in which both $\kappa_{\mathfrak{l}_{1}, \mathfrak{l}_{2}}$ and $\kappa_{\mathfrak{l}_{2}, \mathfrak{l}_{1}}$ play important roles. 


\section{Appendix A}

In this appendix, we prove the following proposition.

Proposition A.1. Suppose that $\mathfrak{r}, \mathfrak{l}$ are two distinct primes in $\mathcal{S}(K)$. We take $\mathfrak{l}^{\prime} \in$ $\mathcal{S}(K(\mathfrak{r}))$ and $b \in\left(K^{\times} \otimes \mathbf{Z}_{p}\right)^{\chi}$ such that $\operatorname{div}(b)=\left(\mathfrak{l}_{K}^{\prime}-\mathfrak{l}_{K}\right)^{\chi}$ and $\phi_{\mathfrak{r}}^{\left(n_{\mathfrak{r}}\right)}(b)=0$ where $\phi_{\mathfrak{r}}^{\left(n_{\mathfrak{r}}\right)}$ : $\left(K^{\times} / p^{n_{\mathfrak{r}}}\right)^{\chi} \longrightarrow O_{\chi} / p^{n_{\mathfrak{r}}}[\Gamma(K / k)] \otimes G_{\mathfrak{r}}$ is the map defined by taking $N=n_{\mathfrak{r}}$. Put $\tilde{\kappa}_{\mathfrak{r}, \mathfrak{l}}=\tilde{\kappa}_{\mathfrak{r}, \mathfrak{l}^{\prime}}-$ $\tilde{\delta}_{\mathfrak{r}} b$. Then $\tilde{\kappa}_{\mathfrak{r}, \mathfrak{l}}$ does not depend on the choice of $\mathfrak{l}^{\prime}$.

Proof. Put $L=K(\mathfrak{r})$ and $G=\operatorname{Gal}(L / K)$. Suppose that we take two primes $\mathfrak{l}_{1}, \mathfrak{l}_{2} \in \mathcal{S}(L)$ such that there exist $b_{1}, b_{2} \in\left(K^{\times} \otimes \mathbf{Z}_{p}\right)^{\chi}$ with $\operatorname{div}\left(b_{i}\right)=\left(\left(\mathfrak{l}_{i}\right)_{K}-\mathfrak{l}_{K}\right)^{\chi}$ and $\phi_{\mathfrak{r}}^{\left(n_{\mathfrak{r}}\right)}\left(b_{i}\right)=0$ for $i=1$, 2. Put $b=b_{1} / b_{2}$. We denote by $K\{\mathfrak{r}\} / K$ the maximal abelian $p$-extension which is unramified outside $\mathfrak{r}$. Consider the $\chi$-component $\operatorname{Gal}(K\{\mathfrak{r}\} / K)^{\chi}$ of the Galois group, and define $K\{\mathfrak{r}\}^{\chi}$ to be the intermediate field such that $\operatorname{Gal}\left(K\{\mathfrak{r}\}^{\chi} / K\right)=\operatorname{Gal}(K\{\mathfrak{r}\} / K)^{\chi}$. We denote by $L^{\prime} / L$ the maximal unramified $p$-extension such that $L^{\prime} / K$ is abelian and $\Delta(K / k)$ acts on $\operatorname{Gal}\left(L^{\prime} / L\right)$ via $\chi$. Then we have a canonical isomorphism $\operatorname{Gal}\left(L^{\prime} / L\right) \stackrel{\simeq}{\longrightarrow}\left(A_{L}^{\chi}\right)_{G}$. Since the ramification index of a prime above $\mathfrak{r}$ in $L / K$ is $p^{n_{\mathfrak{r}}}$ and the ramification index of a prime above $\mathfrak{r}$ in any abelian extension $M / K$ is $\leq p^{n_{\mathfrak{r}}}, L K\{\mathfrak{r}\}^{\chi} / L$ is unramified above $\mathfrak{r}$ and so unramified everywhere. Hence the restriction map

$$
\operatorname{Gal}\left(L^{\prime} / L\right)=\left(A_{L}^{\chi}\right)_{G} \stackrel{\simeq}{\longrightarrow} \operatorname{Gal}\left(K\{\mathfrak{r}\}^{\chi} / K\right)
$$

is bijective.

Put $\Psi=\left(\left(\prod_{v \mid \mathfrak{r}} K_{v}^{\times} / U_{K_{v}}^{1} \times \bigoplus_{v \nmid \mathfrak{r}} K_{v}^{\times} / U_{K_{v}}\right) \otimes \mathbf{Z}_{p}\right)^{\chi}$. We consider the commutative diagram

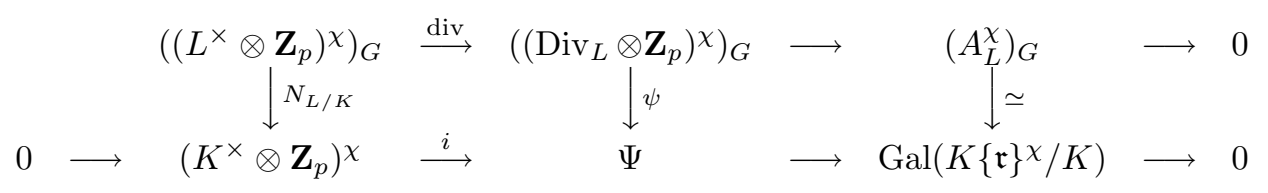

where $i$ is the natural map which is injective because of $\left(O_{K}^{\times} \otimes \mathbf{Z}_{p}\right)^{\chi}=0$, and $\psi$ is the map induced by the norm map. Since $\phi_{\mathfrak{r}}^{\left(n_{\mathfrak{r}}\right)}(b)=0$, the $v$-component of $i(b) \in\left(\left(\prod_{v \mid \mathfrak{r}} K_{v}^{\times} / U_{K_{v}}^{1} \times\right.\right.$ $\left.\left.\bigoplus_{v \nmid \mathfrak{r}} K_{v}^{\times} / U_{K_{v}}\right) \otimes \mathbf{Z}_{p}\right)^{\chi}$ is trivial for all $v \mid \mathfrak{r}$. Hence $i(b)$ is in the image of $\psi$. Therefore, by the above commutative diagram, there is $a \in\left(L^{\times} \otimes \mathbf{Z}_{p}\right)^{\chi}$ such that $N_{L / K}(a)=b$ and $\operatorname{div}(a)=$ $\mathfrak{l}_{1, L}-\mathfrak{l}_{2, L}+\left(\sigma_{\mathfrak{r}}-1\right) x$ for some $x \in\left(\operatorname{Div}_{L} \otimes \mathbf{Z}_{p}\right)^{\chi}$.

We have $\operatorname{div}\left(\left(g_{\mathfrak{l}_{1}}^{L} / g_{\mathfrak{l}_{2}}^{L}\right)\left(\theta_{L}^{\chi} a^{-1}\right)\right)=-\theta_{L}^{\chi}\left(\sigma_{\mathfrak{r}}-1\right) x$. Hence

$$
\operatorname{div}\left(D_{\mathfrak{r}}\left(\frac{g_{\mathfrak{l}_{1}}^{L}}{g_{\mathfrak{l}_{2}}^{L}}\left(\theta_{L}^{\chi} a^{-1}\right)\right)\right)=-D_{\mathfrak{r}} \theta_{L}^{\chi}\left(\sigma_{\mathfrak{r}}-1\right) x=\left(N_{\mathfrak{r}}-p^{n_{\mathfrak{r}}}\right) \theta_{L}^{\chi} x .
$$

By Lemma 2.4, we have $N_{\mathfrak{r}} \theta_{L}^{\chi}=\left(1-\varphi_{\mathfrak{r}}^{-1}\right) \theta_{K}^{\chi} N_{\mathfrak{r}}=0$, hence $\operatorname{div}\left(D_{\mathfrak{r}}\left(\left(g_{\mathfrak{l}_{1}}^{L} / g_{\mathfrak{l}_{2}}^{L}\right)\left(\theta_{L}^{\chi} a^{-1}\right)\right)\right)=$ $-p^{n_{\mathrm{r}}} \theta_{L}^{\chi} x$ holds. We take $g_{x} \in\left(L^{\times} \otimes \mathbf{Z}_{p}\right)^{\chi}$ such that $\operatorname{div}\left(g_{x}\right)=\theta_{L}^{\chi} x$. This is possible because $\theta_{L}^{\chi} A_{L}^{\chi}=0$. Thus, we have $\operatorname{div}\left(D_{\mathfrak{r}}\left(\left(g_{\mathfrak{l}_{1}}^{L} / g_{\mathfrak{l}_{2}}^{L}\right)\left(\theta_{L}^{\chi} a^{-1}\right)\right)\right)=-\operatorname{div}\left(g_{x}^{p^{n_{\mathfrak{r}}}}\right)$, which implies

$$
D_{\mathfrak{r}}\left(\frac{g_{\mathfrak{l}_{1}}^{L}}{g_{\mathfrak{l}_{2}}^{L}}\left(\theta_{L}^{\chi} a^{-1}\right)\right)=g_{x}^{-p^{n_{\mathfrak{r}}}}
$$

in $\left(L^{\times} \otimes \mathbf{Z}_{p}\right)^{\chi}$ by Lemma 3.1. It follows that $D_{\mathfrak{r}}\left(\frac{g_{\mathfrak{t}_{1}}^{L}}{g_{\mathfrak{t}_{2}}^{L}}\right)=D_{\mathfrak{r}} \theta_{L}^{\chi} a$ in $\left(L^{\times} \otimes \mathbf{Z} / p^{N}\right)^{\chi}$. Hence we have in $\left(L^{\times} \otimes \mathbf{Z} / p^{N}\right)^{\chi}$

$$
\frac{\kappa_{\mathfrak{r}, l_{1}}}{\kappa_{\mathfrak{r}, l_{2}}}=D_{\mathfrak{r}} \theta_{L}^{\chi} a=\delta_{\mathfrak{r}} N_{\mathfrak{r}} a=\delta_{\mathfrak{r}} b=\delta_{\mathfrak{r}}\left(\frac{b_{1}}{b_{2}}\right) .
$$


Therefore, we obtain

$$
\frac{\kappa_{\mathfrak{r}, \mathfrak{l}_{1}}}{\delta_{\mathfrak{r}} b_{1}}=\frac{\kappa_{\mathfrak{r}, \mathfrak{l}_{2}}}{\delta_{\mathfrak{r}} b_{2}}
$$

in $\left(K^{\times} \otimes \mathbf{Z} / p^{N}\right)^{\chi}$. This implies $\tilde{\kappa}_{\mathfrak{r}, \mathfrak{l}_{1}}-\tilde{\delta}_{\mathfrak{r}} b_{1}=\tilde{\kappa}_{\mathfrak{r}, \mathfrak{l}_{2}}-\tilde{\delta}_{\mathfrak{r}} b_{2}$, which completes the proof of Proposition A.1.

\section{References}

1. Bourbaki, N., Éléments de Mathématique, Algèbre Commutative, Chap. 7, Hermann, Paris (1965).

2. Deligne, P. and Ribet, K., Values of abelian $L$-functions at negative integers over totally real fields, Invent. math. 59 (1980), 227-286.

3. Ferrero B. and Washington L., The Iwasawa invariant $\mu_{p}$ vanishes for abelian number fields, Ann. of Math. 109 (1979), 377-395.

4. Greither, C., Computing Fitting ideals of Iwasawa modules, Math. Zeitschrift 246 (2004), 733-767.

5. Greither, C. and Kurihara, M., Stickelberger elements, Fitting ideals of class groups of CM fields, and dualisation, Math. Zeitschrift 260 (2008), 905-930.

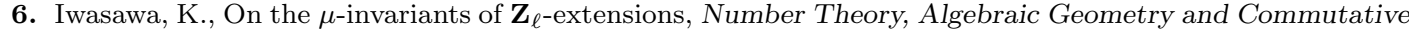
Algebra, in honor of Yasuo Akizuki, Kinokuniya, Tokyo (1973), 1-11; Collected Papers 53, 709-719.

7. Koike, M., On the isomorphism classes of Iwasawa modules associated to imaginary quadratic fields with $\lambda=2$, J. Math. Sci. Univ. Tokyo 6 (1999), 371-396.

8. Kolyvagin, V.A., Euler systems, The Grothendieck Festschrift Vol II (1990), 435-483.

9. Kurihara, M., Iwasawa theory and Fitting ideals, J. reine angew. Math. 561 (2003), 39-86.

10. Kurihara, M., On the structure of ideal class groups of CM-fields, Documenta Mathematica, Extra Volume Kato (2003), 539-563.

11. Kurihara, M., Refined Iwasawa theory for $p$-adic representations and the structure of Selmer groups, preprint.

12. Mazur, B., and Rubin, K, Kolyvagin systems, Memoirs of the AMS Vol 168, Number 799 (2004).

13. Mazur, B. and Wiles, A., Class fields of abelian extensions of Q, Invent. math. 76 (1984), 179-330.

14. Northcott, D. G., Finite free resolutions, Cambridge Univ. Press (1976).

15. Rubin, K., The main conjecture, Appendix to Cyclotomic fields I and II by S. Lang, Graduate Texts in Math. 121, Springer-Verlag (1990), 397-419.

16. Rubin, K., Kolyvagin's system of Gauss sums, Arithmetic Algebraic Geometry, G. van der Geer et al eds, Progress in Math 89 (1991) 309-324.

17. Rubin, K., Euler systems, Annals of Math. Studies 147, Princeton Univ. Press (2000).

18. Schoof, R., The structure of the minus class groups of abelian number fields, Sém. de Théorie des Nombres Paris 1988-89, Birkhäuser, Boston (1990), 185-204.

19. Siegel, C. R., Über die Fourierschen Koeffizienten von Modulformen, Nachr. Akad. Wiss. Göttingen Math.Phys. Kl. II (1970), 15-56.

20. Serre, J.-P., Corps Locaux, Hermann, Paris (1968) (troisième édition).

21. Tate J., Les conjectures de Stark sur les Fonctions L d'Artin en $s=0$, Progress in Math. 47, Birkhäuser (1984).

22. Washington, L., Introduction to cyclotomic fields, Graduate Texts in Math. 83, Springer-Verlag (1982).

23. Wiles, A., The Iwasawa conjecture for totally real fields, Ann. of Math. 131 (1990), 493-540.

24. Wingberg, K., Duality theorems for $\Gamma$-extensions of algebraic number fields, Compos. Math. 55 (1985), 333-381.

Masato KuRIHARA

Department of Mathematics,

Keio University,

3-14-1 Hiyoshi, Kohoku-ku,

Yokohama, 223-8522, Japan

kurihara@math.keio.ac.jp 